Portland State University

PDXScholar

$1-1-1978$

\title{
Memory for crossed and nested classifications
}

John Ernest Garwood

Portland State University

Follow this and additional works at: https://pdxscholar.library.pdx.edu/open_access_etds

Part of the Cognitive Psychology Commons

Let us know how access to this document benefits you.

\section{Recommended Citation}

Garwood, John Ernest, "Memory for crossed and nested classifications" (1978). Dissertations and Theses. Paper 843.

https://doi.org/10.15760/etd.843

This Thesis is brought to you for free and open access. It has been accepted for inclusion in Dissertations and Theses by an authorized administrator of PDXScholar. Please contact us if we can make this document more accessible: pdxscholar@pdx.edu. 
MEMORY FOR CROSSED AND NESTED

CLASSIFICATIONS

by

JOHN ERNEST GARWOOD

A thesis submitted in partial fulfillment of the requirements for the degree of

MASTER OF SCIENCE
in
PSYCHOLOGY

\author{
Portland State University \\ 1978 \\ (C) 1978 John Ernest Garwood
}


TO THE OFFICE OF GRADUATE STUDIES AND RESEARCH:

The members of the Committee approve the thesis of

John Ernest Garwood presented January 27, 1978.

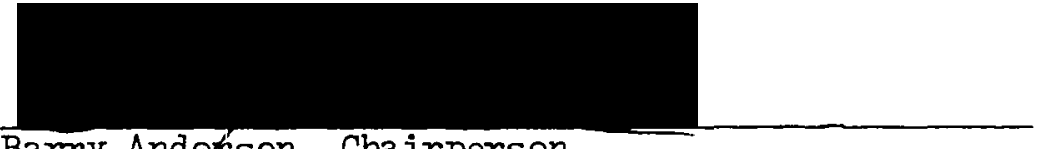

Barry Anderson, Chairperson
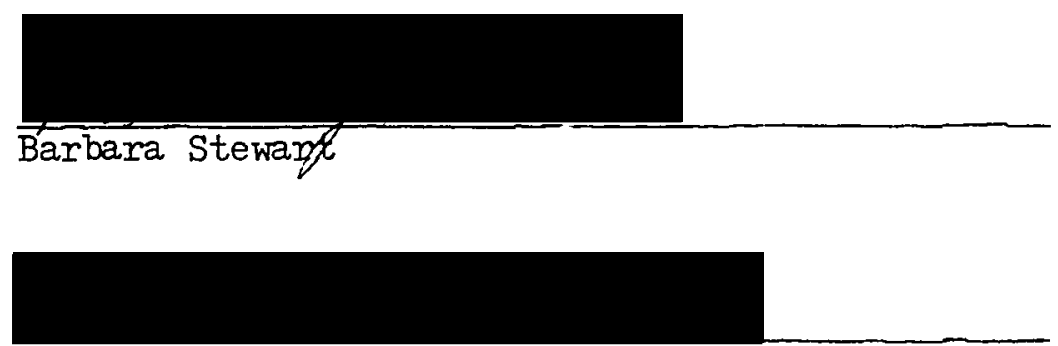

Morris Weitman

APPROVED :
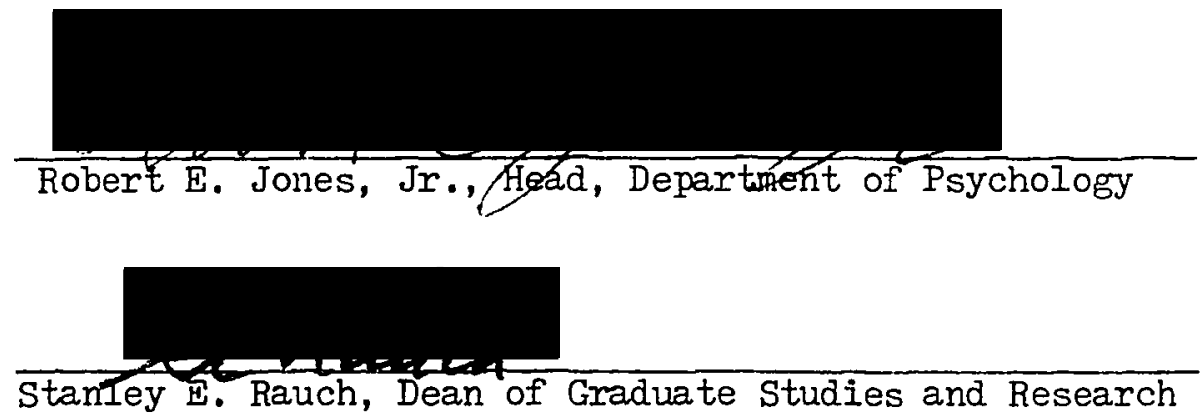
AN ABSTRACT OF THE THESIS OF John Ernest Garwood for the Master of Science in Psychology presented January 27, 1978.

Title: Memory for Crossed and Nested Classifications.

APPROVED BY MEMBERS OF THE THESIS COMMITTEE:

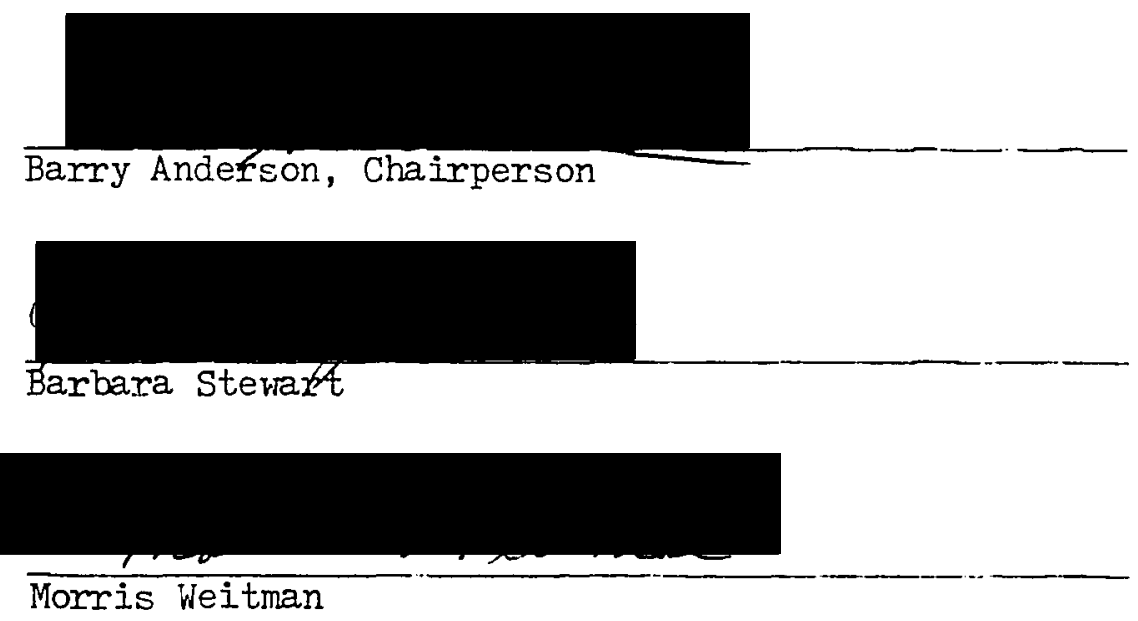

Memory for crossed and nested classifications was investigated. Two experimental groups were exposed to stimuli which could be organized by both a crossed and nested classification. The stimuli consisted of nine drawings in a $3 \times 3$ matrix. Each drawing is characterized by attributes on five dimensions. The nested classification requires four dimensions to organize the nine drawings, while the crossed classification requires two dimensions. Of the five dimensions, three are unique to the nested classification, one is unique to the crossed classification, and one is common to both classifications. 
Subjects were presented the stimuli so that either the crossed or nested aspects were emphasized. This emphasis was accomplished by manipulating both the temporal order of rehearsal and the physical format, which were confounded in this experiment. Both nested and crossed groups first rehearsed the common dinension. The crossed group secondly rehearsed the other crossed dimension and thirdly the three nested dimensions. The nested gxoup secondly rehearsed the three nested dimensions, then thirdly the crossed dimension. Also, the physical format for the two groups differed by having different lines separating the drawings of their sets.

Three measures were taken during the recall of the sets. The temporal order of recall of the stimulus attributes was noted. Errors were counted when the attributes of any dimension were recalled in the wrong structural location, or were not recalled at all. Clustering was measured for each recall trial. Clustering is the tendency to recall all of one dimension, then all of another dimension, etc., rather than mix dimensions together.

It was hypothesized (1) that subjects in the nested group would make fewer errors in the recall of the stimuli than would the crossed subjects; (2) that the crossed group would cluster in their recall to a greater extent than the nested group, and (3) that the two presentation methods would induce the subjects to record in long-term memory their respective structures better than the structure of the other group.

Twenty four Portland State University undergraduates 
participated in the study. The error hypothesis (1) was not confirmed. For short-term memory, both groups made the same number of errors. The clustering hypothesis (2) was not confirmed. The induction of structure hypothesis (3) was partially confirmed. The nested group recalled the nesting with fewer errors than the cross. The crossed group recalled both structures equally poorly. A learning curve hypothesis (4) was not confirmed. A subject expectation effect hypothesis (5) was partially confirmed. Subjects in both groups increased their errors when they tried to rearrange the material in their memory in certain ways before recall.

Several post-hoc analyses were performed. For short-term recall, errors were correlated with absolute rearrangement. Absolute rearrangement measures the difference between dimensional position in the presentation and recall sequences. The position change of the dimensions unique to the nested classification was found to correlate significantly with errors for both groups.

The experimental hypotheses and post-hoc analyses are consistent with the hypothesis that the nested classification tends to override the cross classification when the two are in direct competition. Overall, the experimental results provide support for the hypothesis that the experimental procedure can induce the memory of nested classifications far better than the memory of cross classifications. 
ACKNOWLEDGENENTS

I would like to thank first, and most appreciatively, Barry Anderson. It was he who suggested to me the subject of this thesis. His patient guidance, encouragement, experimental acuteness, and enthusiasm have sustained me throughout the thesis. I would like to extend my thanks to Morris Weitman for his encouragement and his high methodological standards, which helped me refine the experimental procedures. Barbara Stewart has my appreciation for her critical acuity, her thorough statistical knowledge, and her warmth in moments of stress. I could not have had a better committee.

I would like to thank the PSU Psychology Clinic under Joan Behn and Barbara Mecca for the use of their facilities. I am most grateful for the 24 psychology students who participated in the experiment. Without their cooperation, this thesis simply could not have been done. Iast of all I would like to thank Ann Sarah DuFresne for her special kind of support. 
ACKNOWLEDGEMENTS .................. . . . . iii

LIST OF TABLES ................... . . . viii

LIST OF FIGURES . . . . . . . . . . . . . . . . $\mathrm{x}$

CHAPTER

I INTRODUCTION . . . . . . . . . . . 1

Crossed and Nested Classifications . . . 1

An Example of a Crossed Classification

An Example of a Nestel Classification

A Nested Classification in Science

Crossed Classification in the Scientific Method

Nested Classification and the Structure of Semantic Memory

Memory Efficiency and the Definitions . .

The Question of Memory Efficiency

The Definition of Crossed Classification

The Definition of a Nested classification

The Theoretical Comparison of Memory Efficiency

The Experimental Comparison . . . . . .

Experimental Approaches to Comparison

The Final Design: The Temporal

Manipulation of Structure

The Structurally Ambiguous Stimulus Set

The Comparison Design

The Test of the Hypothesis of the Temporal Induction of Structure

The Error Measure 
The Clustering Measure

Hypotheses . . . . . . . . . . .

The Error Hypothesis

The Clustering Hypothesis

The Learning Curve Hypothesis

The Subject Expectation Effect

Summary Hypothesis

II

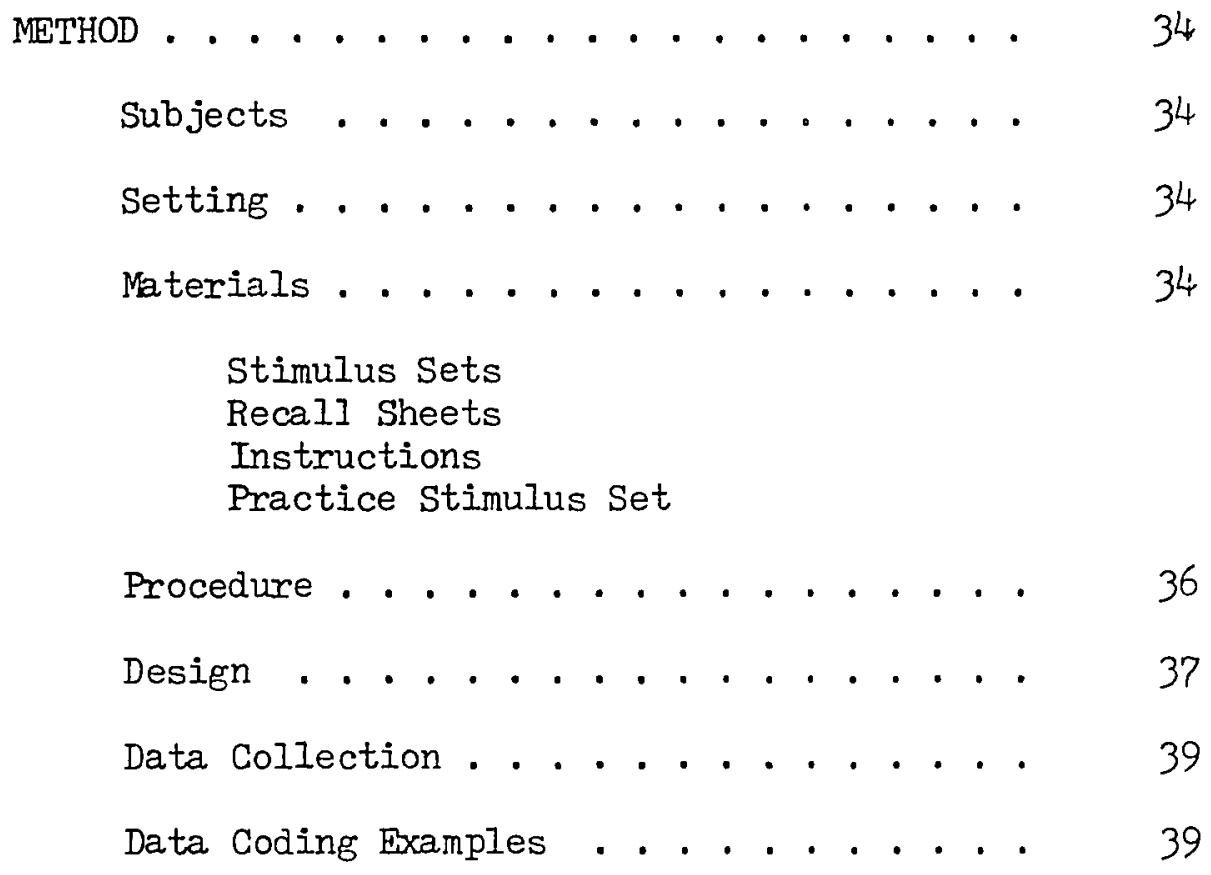

III Results

Results of Experimental Hypotheses . . .

(1) Error Hypothesis

(2) Clustering Hypothesis

(3) Learning Curve Hypothesis

(4) Induction of Structure Mypothesis

(5) Subject Expectation Effect Hypothesis

Post-hoc Analysis . . . . . . . . . .

Total Errors, Long-term Recall Stmuctural Errors, Short-term Recall Absolute Rearrangement 


$$
\begin{aligned}
& \text { Absolute Rearrangement Error } \\
& \text { Correlations for Short-term } \\
& \text { Recall } \\
& \text { Rearrangenent Error Correlations for } \\
& \text { Long-term Recall }
\end{aligned}
$$$$
\text { Subjects' Self-reports . . . . . . } 58
$$

Experimental Hypotheses . . . . . . 59

(1) Error Hypothesis

(2) Clustering Hypothesis

(3) Learning Curve Hypothesis

(4) Induction of Structure Hypothesis

(5) Subject Expectation Effect

Post-hoc Analysis . . . . . . . .

Absolute Rearrangement Error

Correlations for Short-term Recall

Rearrangement Error Correlations for Long-term Recall

Total Errors, Long-term Recall

Structural Errors, Short-term Recall

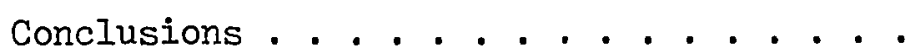

Implications for Further Research . . . .

Amplification

Clarification

Extension

REFERENCES . . . . . . . . . . . . . . . . .

A EXPERIMENTAL INSTRUCTIONS . . . . . . . .

B PRESENTATION ORDERS ............. 
E PRACTICE STIMULI CONTENT MODES, LABELS, CONTENT MODE ROTATIONS AND STIMULUS SETS . . . . 83

F STIMULUS CONTENTS, CONTENT MODES, LABELS, ROTATIONS, AND STIMULUS SETS . . . . . 
LIST OF TABLES

TABLE

PAGE

I The Five Mountain Stimulus Sets . . . . . .

II Assignment to Subjects of Stinulus Sets by

Content Mode, Trial Order, and Set Content

III Summary of Analyses . . . . . . . . . .

44

IV ANOVA for Error Data, Using Conservative Degrees of Freedom ...............

$\mathrm{V}$ ANOVA for the Arcsin Transform of the Clustering

Data, Using Conservative Degrees of

Freedom . . . . . . . . . . 46

VI Post-hoc Comparison on the Trials Factor of the

Clustering Data by the Newman-Keuls

Procedure .. . . . . . . . . .

VII Long-term Recall Error Data, Means, Standard

Deviations, and $\underline{t}$-test values for

Differences ..............

VIII Pearson Product-moment Correlations Between $\mathrm{R}_{\mathbf{i}}$ and Errors ..............

IX ANOVA for Short-term Recall Column Erroxs Using Conservative Degrees of Freedom . . . . .

$X \quad$ ANOVA for Short-term Recall Row Errors Using Conservative Degrees of Freedom . . . . . 
XI ANOVA for Short-term Recall Within-column Errors Using Conservative Degrees of Freedom . . .

XII Pearson Product-moment Correlations Between

Total Errors and Absolute Rearrangement for Short-term Recall . . . . . . . 56

XIII Pearson Product-moment Correlations Between

Total Errors and Rearrangement Measures for Long-term Recall . . . . . . . 57

XIV Pearson Product-moment Correlations Between

Total Errors and Absolute Rearrangement For Long-term Recall . . . . . . . 57

XV Practice Stimuli Content Modes and Labels . . . 83

XVI Practice Stimuli Content Mode Rotations . . . 83

XVII Stimulus Content Modes, Labels, Rotations . . 90 


\section{IIST OF FIGURES}

FIGURE

PAGE

1 The Nested Classification in a Table of Contents

2 Eight of the Principles which Subdivide the

Kingdom Plantae into Divisions . . . .

3 Twelve of the Principles which Subdivide the

Kingdom Animalia into Phyla . . . . . . 5

4 Cloud Seeding Fourfold Table . . . . . . . 7

5 Single Row Data of Cloud Seeding Table . . . . 8

6a Rainy Clinate ............ 8

6b Dry Climate ............... 8

7 Reaction Times to the Canary Question . . . . 9

8 The Hypothetical Menory Structure for a Three

LeveI Hierarchy . . . . . . . . . . 10

9 A Crossed Classification ......... 12

10 A Nested Classification ........... 13

11 An Ambiguous Stimulus Set . . . . . . . 17

12 Data Grid . . . . . . . . . . 21

13 An Example of Minimum Clustering . . . . . . 41

14 Minimum Clustering on a Data Grid . . . . . 41

15 An Example of Maximum Clustering ........ 42

16 Maximum Clustering on a Data Crid ....... 42 
17 Scatter Diagram and Regression Line for

Crossed Group Error Data $\left(E_{C}\right)$ and the

Second Rearrangement Measure $\left(R_{2}^{c}\right)$. . . .

18 Scatter Diagram and Regression Line for

Crossed Group Error Data $\left(E_{c}\right)$ and the

Third Rearrangement Measure $\left(\mathrm{R}_{3}^{\mathrm{c}}\right) \ldots .$.
Nested Group Error Data $\left(\mathrm{E}_{n}\right)$ with the
Second Absolute Rearrangement Measure $\left(\mathrm{A}_{2}^{\mathrm{n}}\right)$

and with the Within-column Dimension First

Appearance $\left(w^{n}\right)$........... 64

20 Scatter Diagram and Regression Line for

Crossed Group Error Data $\left(\mathrm{E}_{C}\right)$ with the

Third Absolute Rearrangement Measure $\left(A_{3}^{c}\right)$

and with the Within-column Dimension First

Appearance $\left(w^{c}\right)$. . . . . . . . 
CHAPTER I

INTRODUCTION

CROSSED AND NESTED CLASSIFICATIONS

Crossed and nested classifications are important in everyday thought, in scientific thinking, and in the psychology of memory. These two ways of organizing information are prominent in many areas of our lives. As such they are worthy of experimental investigation. A review of the literature failed to produce any studies in which these two classifications were experimentally compared. The purpose of this study is to begin the experimental comparison of these two classifications.

An example of a crossed classification

An example of a crossed classification is the blank form students use to lay out their class schedules. The form is usually a five-by-eleven grid. Across the top of the grid are listed the days of the week from Monday through Friday. Each column of the grid corresponds to one of the days. Along the side of the grid are usually listed the hours of the day from 8 A.M. to ? P.M. Each row of the grid corresponds to a one-hour block of iime. The grid represents all combinations of the days of the week and the times of the day. Students can use this grid as a flexible tool to organize their class schedules, resolve conflicts between classes meeting at 
the same time, and remember their schedules. The class schedule form is one example of a pragmatic use of the crossed classification.

An example of a nested classification

Common, everyday instances of nested classifications can be found in the tables of contents of many non-fiction books. For example, consider the table of contents of The April Game by Diogenes (see figure 1), a book written by an anonymous Internal Revenue Service agent about his pithy inside view of the Internal

I. The People Versus The Tax

1. The Game

2. The Revenue and the Agent

3. Rich Man, Poor Man, Middleman...

4. . . Thief

5. Through History With the Tax Man

II. The People Under, Around, Over and Through the Tax

6. The Backward-Graduated Tax

7. The Secret River

8. Bribery and the Revenue Agent

9. The Revenue Service: An American Gestapo?

10. A Softhearted Gestapo, Anyway

11. The Tax Informers

12. The Tax and the Society

III. You And Me Against the Tax

13. How To Be Audited and Come Out Alive

14. The Middle-Income Taxpayer's Cheating Guide

15. Is There a Better Tax than the Income Tax?

Figure 1. The nested classification in a table of contents (From Diogenes. The April Game. Playboy Press, Chicago, Illinois, 1973, pp. v-vi).

Revenue Service. The table of contents is divided into three sections. Each section is further divided into chapters. Comparison of the chapters of one section with those of another will show that none of the chapters duplicates any other. Each section is subdivided on a different basis. This is a key characteristic of a nested 
classification. Once material is divided, any further subdivision is on a different basis in each subdivision. This is an everyday example of a nested classification.

A nested classification in science

An example of a nested classification in science is the biological taxonomic system (Levy, 1973). All living things are divided into four kingdoms, Monera, Protista, Plantae, and Animalia. The kingdom Plantae is composed mostly of multicellular eucaryotes (containing distinct organelles or subcellular specialized structures), most of which are photosynthetic. The kingdom Animalia consists of multicellular eucaryotes which are heterotrophic (directly dependent on producers for their food). These two kingdoms are distinguished from each other with few exceptions by their ability or inability to produce their own food directly from non-living elements.

These two kingdoms can be further divided into subclasses. The Plantae kingdom has two subkingdoms. One subkingdom has four divisions, the other ten subdivisions. All told, the Plantae kingdom is divided into 14 divisions. One of the principles which helps distinguish one division from another is the "flower and fruit" principle. Only one division, the Anthophyta, contains plants which produce fruits and flowers (Levy, 1973, p. 322). Levy lists eight principles (see figure 2) which subdivide the kingdom Plantae into divisions.

The kingdom Animalia is subdivided into 20 phyla. One of the 
principles which divides one phylum from another is the "segmentation" principle (Levy, 1973, p. 337). For example, lobsters have a segmented exoskeleton, while snails have a solid shell. Levy lists 12 principles (see figure 3) which subdivide the kingdom Animalia into phyla.

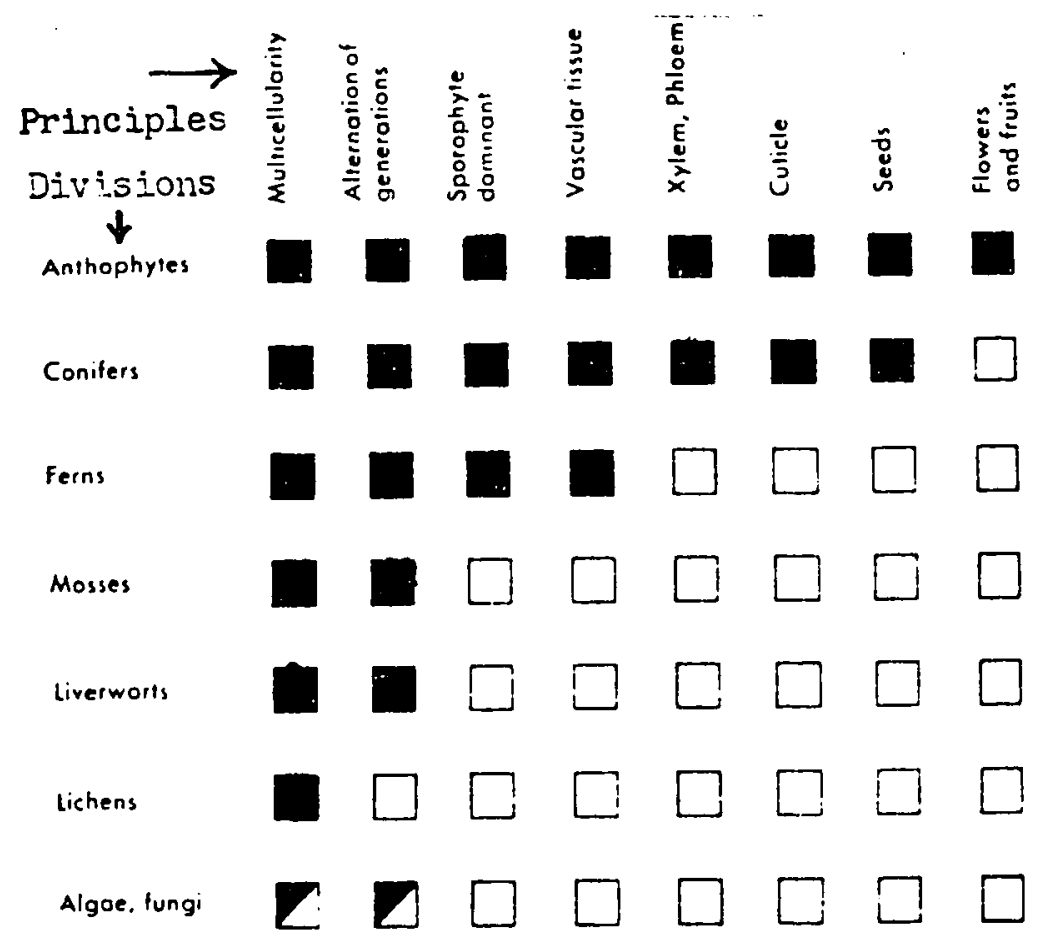

Figure 2. Eight of the principles which subdivide the kingdom Plantae into divisions (From Charles $\mathrm{K}$. Levy. Elements of Biology. Appleton-Century-Crofts, Educational Division, Meredith Co., N.Y., N.Y., 1973, p. 322).

These divisions and subdivisions are arraned in a nested classification. The kingdoms Plantae and Animalia are mostly divided by the distinction between direct and indirect food production. Once this distinction has been made, the two kingdoms are further divided by a number of principles. If you compare the two sets of principles in figure 2 and figure 3, you will see that 


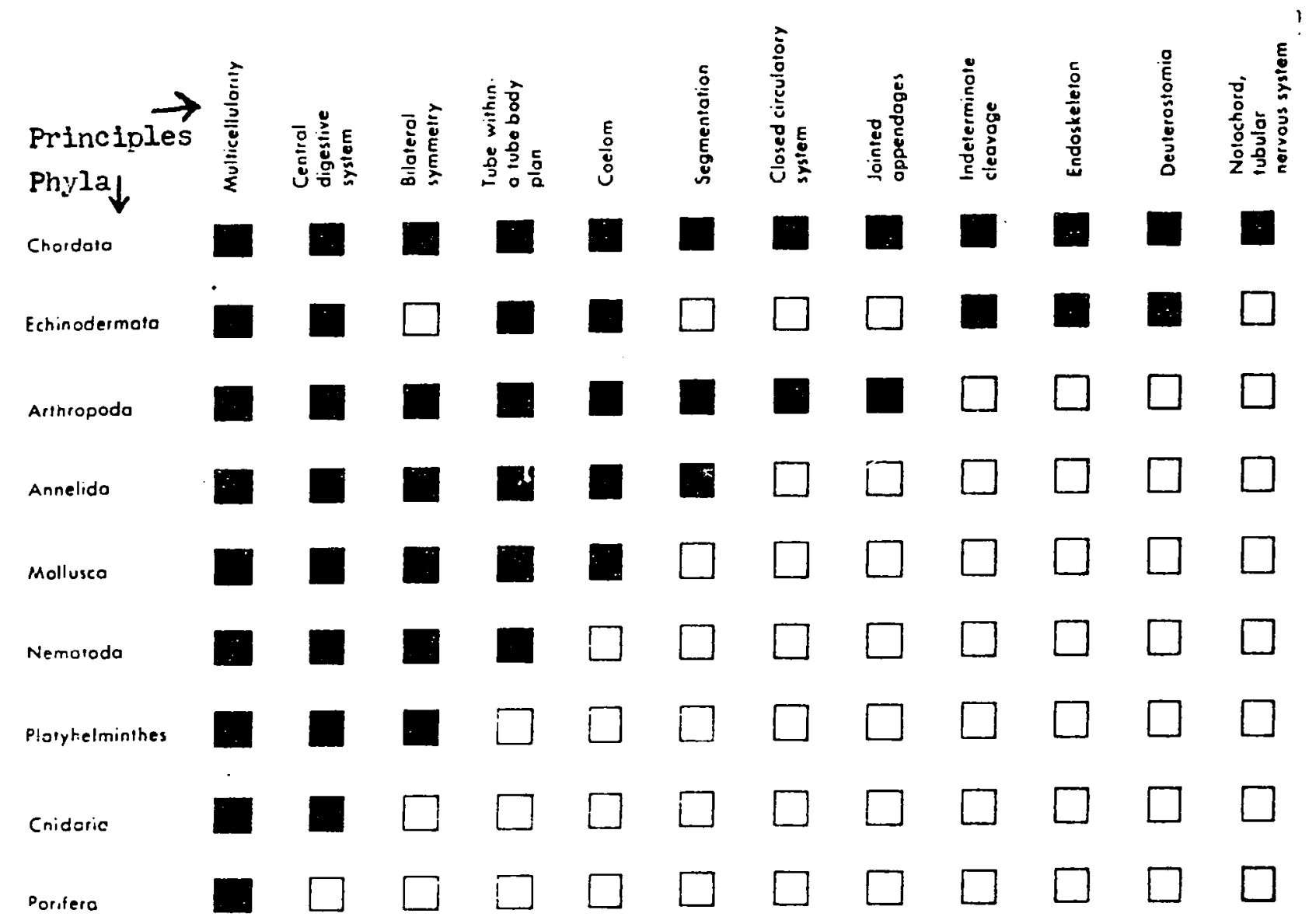

Figure 3. Twelve of the principles which subdivide the Kingdom Animalia into phyla (Fion Division, Meredith Co., N.Y., N.Y., 1973, p. 337). 
there is no duplication. That lack of duplication is the hallmark of the nested classification. Once a set has been divided, the subsets each have their own separate principles for further subdivision. There exists no duplication between the principles of each subset. The biological taxonomic system is a prime example of a nested classification in science.

Crossed classification in the scientific method

Crossed classification is an indispensable part of the scientific method. Its most elementary form is the fourfold table (Anderson, 1971, pp. 16-21). The fourfold table is constructed by crossing two variables, each of which has two states. As an example, let us consider a cloud seeding experiment. This example will first be presented as an incorrectly designed experiment, that is, as an experiment that does not nroperly use the fourfold table. Then the correct procedure using the crossed classification of the fourfold table will be presented.

Suppose you were testing a particular method of cloud seeding, one which uses silver iodide particles. You would like to know if seeding with silver iodide causes rain. As a careful experimenter, you keep track of the days of rain and days of no rain after the clouds are seeded. Suppose further that out of 90 days of seeding, it rained 81 days and did not rain 9 days. As an experimenter, you carefully compute a ratio of rainy days to days of no rain after seeding. Since the ratio is $9: 1$, you conclude that silver iodide seeding does indeed cause rain. 
Alas, it is not correct to make such a conclusion. What are lacking are control data. In particular, when no seeding is done, what is the proportion of rainy days to days without rain? Suppose your plane had to be repaired for 10 days during the experiment so that no seeding took place on those days. If you checked the rain data for those days, you could get some control data. Suppose you discovered that it rained on 9 days and did not rain on 1 day. That ratio is also 9:1, which strongly suggests that you are doing your experiment in a rainy climate. On the other hand, if it rained on 1 day and did not rain on 9, that strongly suggests your climate is a dry one, which greatly strengthens the conclusion that your cloud seeding method causes rain.

A simple way of summarizing the data is in the crossed classification of the fourfold table. Let variable one be the cloud seeding variable, and variable two be the rain variable. The resulting fourfold table is illustrated in figure 4. As first presented, the cloud seeding experiment focused on the data of a single row, as illustrated in figure 5. This incorrect design is

rain

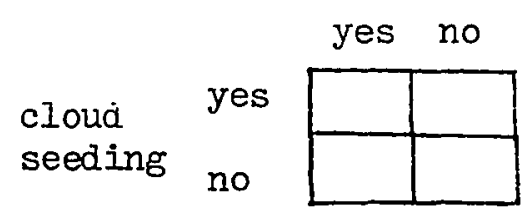

Figure 4. Cloud seeding fourfold table

known as the single row fallacy (Anderson, 1971, pp. 17, 19). There are other fallacies, known as the single cell, main diagonal, 
and the marginal fallacies (Anderson, 1971, pp. 17-21).

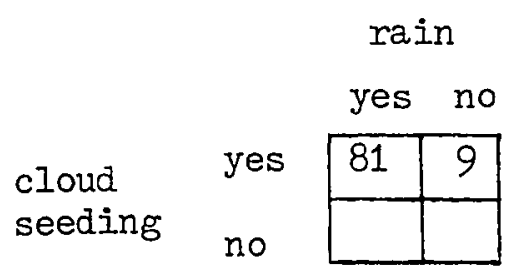

Figure 5. Single row data of cloud seeding table

When we include the control data, our crossed classified fourfold table looks like figures $6 \mathrm{a}$ and $6 \mathrm{~b}$. From the full data of the

\begin{tabular}{|c|c|c|c|c|c|c|c|}
\hline \multirow{4}{*}{$\begin{array}{l}\text { cloud } \\
\text { seeding }\end{array}$} & \multicolumn{3}{|c|}{ rain } & & & \multicolumn{2}{|c|}{ rain } \\
\hline & & yes & no & & & yes & no \\
\hline & yes & 81 & 9 & cloud & yes & 81 & 9 \\
\hline & no & 9 & 1 & seeding & no & 1 & 9 \\
\hline
\end{tabular}

fourfold crossed classification, decisions concerning causal links can be made since the independent variable (cloud seeding) was manipulated. For example, a phi coefficient could be calculated and interpreted (Hays, 1973, pp. 742-745). More complex experimental designs are generalizations of the primitive fourfold table. Crossed classification is one of the bases of the scientific method.

Nested classification and the structure of semantic memory Nested classifications also make their appearance in studies of the organization of semantic memory. Collins and Quillian (1969) asked subjects questions about the properties of animals, such as "Does a canary have a skin?" and "Can a canary sing?". 
They measured the time subjects took to react to these questions. Longer reaction times were recorded for the skin question than for the singing question. This suggests that skin is not as directly associated with canaries as is singing. Likewise for superordinate classes to which canaries belong, reaction time for more inclusive classes such as animals was longer than the reaction time for less inclusive classes (see figure 7).

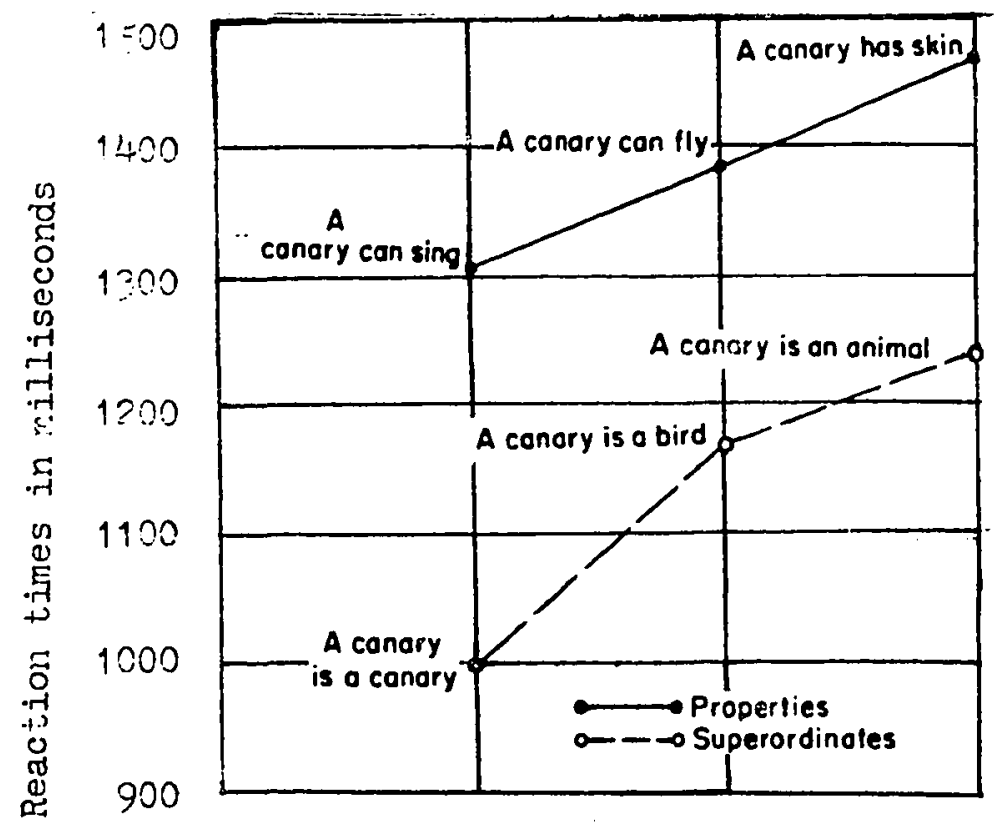

Figure 7. Reaction times to the canary question (adapted from Collins \& Quillian, 1969, p. 244).

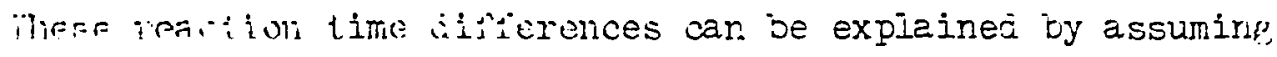
the concept of a canary is part of a nested classification or hierarchy (see figure 8). Each underlined noun in figure 8 represents a class name, such as "canary", "bird", or "animal". Each step in the classification has an incremental reaction time attached to it. Collins and Quillian found subjects took an average 
of 75 milliseconds to move from class to class and an average of 225 milliseconds to move from class to property (Collins \& Quillian, 1969, p. 245). The total reaction time is the sum of the basic recognition time of 1000 milliseconds and the incremental times of the steps between the concept and the correct property. A nested classification can account for the reaction times to questions about the properties of animals. This experiment can be interpreted as evidence for the hypothesis that at least some of semantic memory is organized into a nested classification.

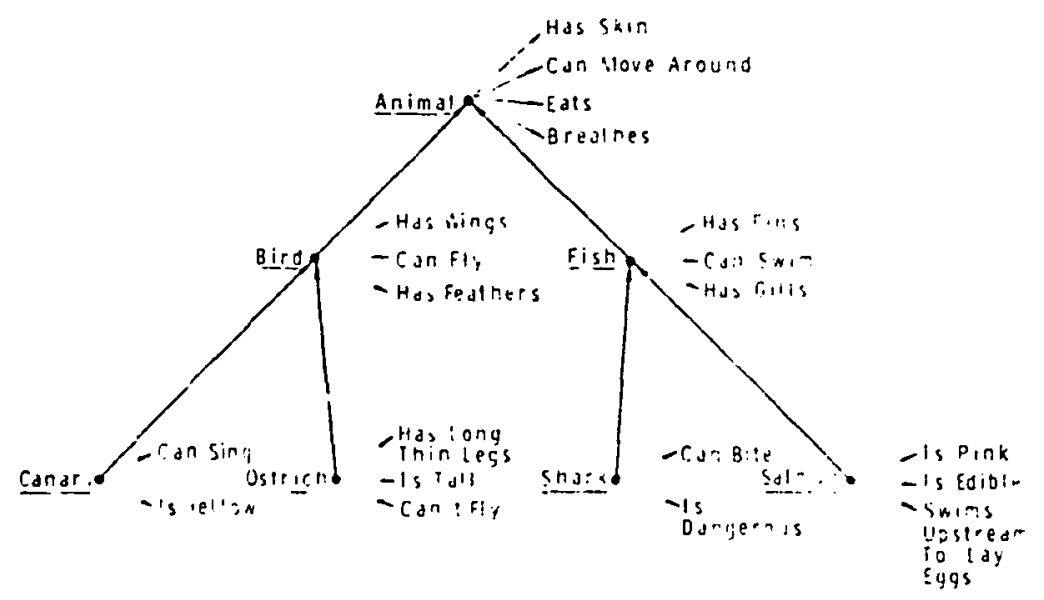

Figure 8. The hypothetical memory structure for a three level hierarchy (Collins \& Quillian, 1969, p. 241)

MEMORY EFFICTENCY AND THE DEFINITIONS

The question of memory efficiency

If semantic memory is organized into a nested hierarchy, is that the most efficient type of structure? We can begin by making a theoretical comparison of memory efficiencies between the two types of organization. We can make this comparison on the basis of the number of discrete items of information needed to define each 
classification completely. In order to make this comparison, we need to define crossed and nested classifications precisely.

The definition of crossed classification

For our purposes, we need only define a crossed classification for a set of nine objects using two dimensions. Let the two stimulus dimensions be called "A" and "B". Let each stimulus dimension have three attribute states. For $A$, these attribute states are $A_{1}, A_{2}$, and $\mathrm{A}_{3}$. For $\mathrm{B}$, these attribute states are $\mathrm{B}_{1}, \mathrm{~B}_{2}$, and $\mathrm{B}_{3}$. As a shorthand convenience, call each attribute state an "attribute". A crossed classification is constructed from all the possible combinations that the attributes of the two dimensions can make with each other. The three attributes of $A$ and $B$ make a total of nine distinct combinations. Each combination corresponds to and describes one of the nine objects.

Let these nine combinations be arranged into a $3 \times 3$ matrix in a particular order. Let the three combinations with $B_{1}$ be in the first column, the three with $B_{2}$ in the second column, and the three with $B_{3}$ in the third column. Similarly, let the three combinations with $A_{1}$ be in the first row, the three with $A_{2}$ in the second row, and the three with $\mathrm{A}_{3}$ in the third row. This arrangement is illustrated in figure 9. Let B be called the "column dimension", because the $B$ attributes distinguish between columns but not between rows. Let $\mathrm{A}$ be called the "row dimension" because A's attributes distinguish between rows but not between columns. The important characteristic is that each attribute appear in 
only one row or column and in no other row or column. The particular

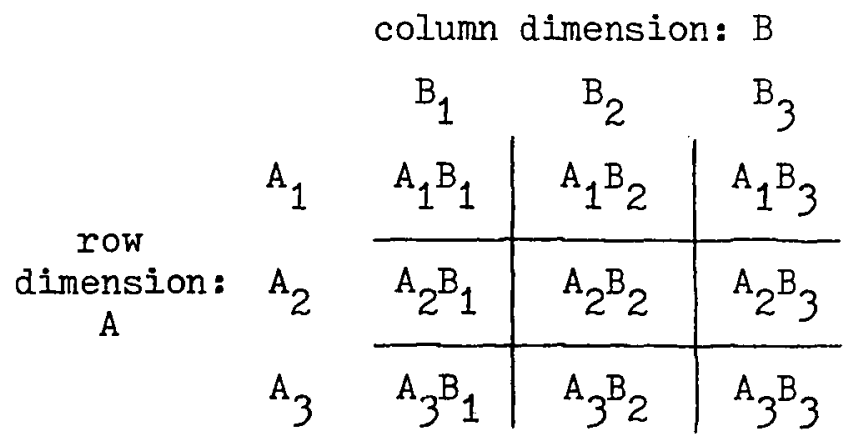

Figure 9. A crossed classification

order of the attributes from column to column (or row to row) is of no significance for the definition of a crossed classification. As an example, $B_{1}$ may be in the second column with $E_{2}$ in the first, and the structure of the nine combinations will still conform to the definition of a crossed classification. A crossed classification has two characteristics. Each attribute on either dimension is combined with all attributes on the other to make all possible combinations. For any attribute, the combinations containing that attribute must all appear in either a single row or a single column. These two conditions completely define a two-dimensional crossed classification.

This definition gives us a simple way to measure the minimum number of items necessary to reproduce the crossed classification. The minimum number of items is equal to the number of attributes. For our case of nine objects in a $3 \times 3$ crossed classification, there are six attributes, three from each of the two dimensions. The recall of the $3 \times 3$ crossed classification requires the 
memorization of six items, plus the rule for combining them.

The definition of a nested classification

Again, for our purposes, we need only to define a nested classification for a set of nine objects. In contrast to the crossed classification, we will need four dimensions. Iset the four stimulus dimensions be called "B", "C", "D", and "E". Let each of the dimensions have three attribute states, e.g. $B_{1}, B_{2}, B_{3}$, etc. Call B the "main dimension" and its attributes the "main attributes". Call C, D, and E the "subordinate dimensions" and their attributes the "subordinate attributes".

The main and subordinate attributes must be combined in a particular fashion for the combination to be a nested classification. This pattern is illustrated in figure 10. Again we are dealing with

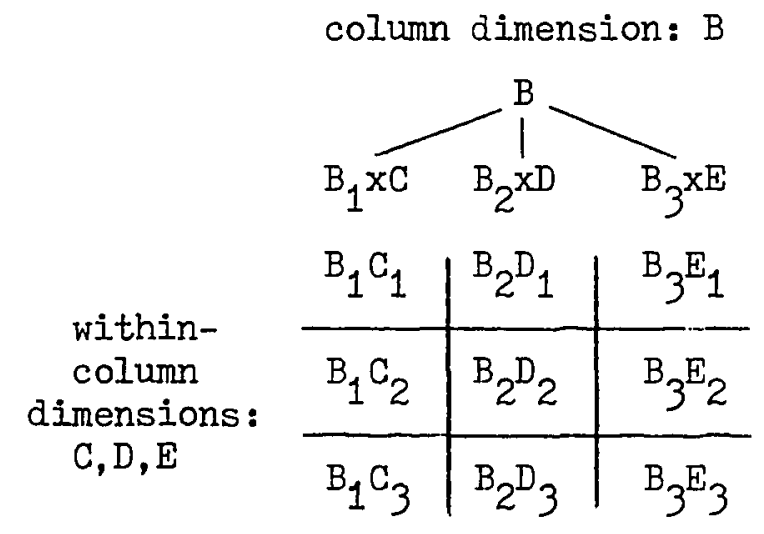

Figure 10. A nested classification

nine comtinations of attributes. Dimension C, D, and E attributes are not combined with each other. Instead, the attributes of each are combined with one of the attributes of the main dimension, and only one. Thus $B_{1}$ is combined with the three attributes of $C$ to 
form the three combinations of column one of the nine item nesting. Similarly, $B_{2}$ is combined with the three attributes of $D$ to form the elements of column two. $B_{3}$ is combined with the three attributes of $\mathrm{E}$ to form column three. These combinations form the pattern of the nested classification.

It can be seen that the main dimension could just as well divide the rows, where the subordinate dimensions would appear only in one row. For our purposes, we need consider only the case where the main dimension divides the columns, etc. Let the main dimension be called the "column dimension". Let the subordinate dimensions be called the "within-column dimensions".

The important characteristic is that each of the within-column dimensions appears in one column and in no other. The particular column in which a within-column dimension appears is of no significance for the definition of a nested classification. Each of the column attributes must also appear in only one column. Likewise, the particular column in which the column attribute appears is of no significance. The necessary condition for a nine--item nesting is that each attribute of the column dimension appear in combination with the attributes of only one of the within-column dimensions. This condition defines the nested classification.

The minimum number of items necessary to reproduce the nested classification can be calculated easily. Each dimension has three attributes, and there are four dimensions. The minimum number is thus twelve items. The recall of our nine object nesting requires the memorization of 12 discrete items, plus the mule for combining 
them.

The theoretical comparison of memory efficiency

We can now compare the theoretical memorization efficiencies of the crossed and nested classifications. Their theoretical efficiencies are given by the minimum number of items necessary for the reproduction of each classification. This comparison shows the crossed classification to be more efficient than the nested, since the crossed needs six items, whereas the nested needs twelve items, to code nine objects with maximal efficiency. On this basis, we could speculate that the crossed classification should be more effective for memorization than the nested.

THE EXPERIMENTAL COMPARISON

\section{Experimental approaches tn comparison}

This theoretical efficiency comparison, along with the phenomena cited, shows the need for the study of crossed and nested classifications. One of the ways of studying these classifications is to compare them experimentally. Experimental comparison proved to be an elusive goal. Several designs were constructed and discarded before the design utilized in this thesis was created.

One design involves the creation of a truly ambiguous matrix, one that can be seen as either crossed or nested following the proper prompting. Experimentation with numerical stimuli proved unrewarding, as did several other approaches along this line. For example, matrices were constructed by adding and multiplying whole 
numbers. The problem with these matrices was that they were either obviously crossed or obviously nested. In other words, one structure strongly dominated the matrix, even though both structures were present. Perhaps some later experimenter will be able to find a truly ambiguous matrix using numerical stimuli.

The final design: the temporal manipulation of structure

The final design presents the stimulus set to the subjects in an order that emphasizes either the crossed or the nested aspects of the stimulus set. Instead of manipulating numbers, this design manipulates the temporal order of non-numerical elements of a stimulus set. This procedure requires a structiurally ambiguous stimulus set that contains both a nested anc a crossed classification. One set of subjects is shown the structurally ambiguous set so that the crossed structure is emphasized (a) by the order of presentation (to be described later) and (b) by lines of equal thickness on the stimulus set (see Appendix D). The other group of subjects is shown the structurally ambiguous set so that the nested structure is emphasized (a) by the order of presentation (to be described later) and (b) by heavy lines seperating the columns of the stimulus set (see Appendix D).

Comparison of these two groups will be comparison of the crossed and nested stmctures as differentiated by the presentation orders and the physical formats of the structurally ambiguous stimulus sets. This comparison does confound presentation order and physical format as independent variables. However, more importantly, this 
comparison does not confound structural type with information load because the two groups will be recalling the same material.

The structurally ambiguous stimulus set

The structurally ambiguous stimulus set, hereafter called simply the ambiguous set, is composed of five dimensions arranged in a $3 \times 3$ matrix. We can use the dimensional definitions established earlier to describe this ambiguous set. The ambiguous set is composed of one row dimension (A), one column dimension (B), and three within-column dimensions ( $C, D$, and $E$ ). The structure of the ambiguous set is illustrated in figure 11. The ambiguous set contains both a nested and a crossed classification. The row dimension (A)

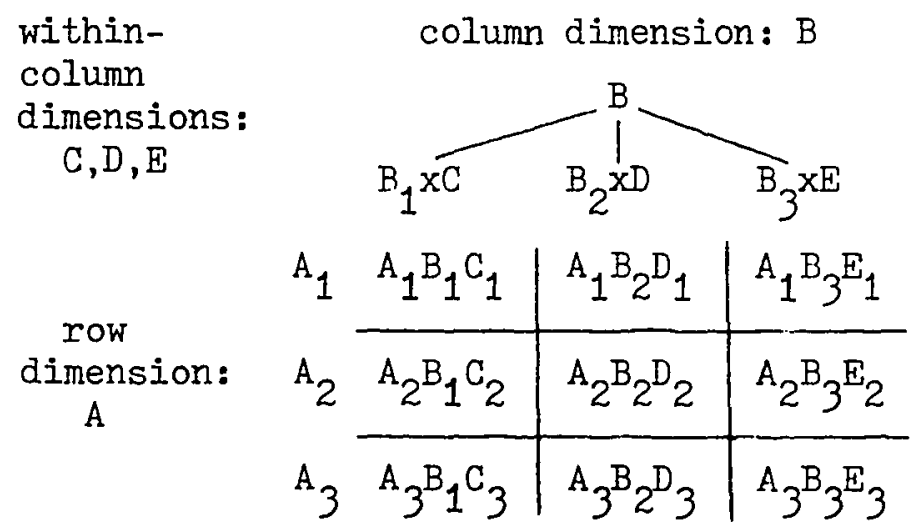

Figure 11. An ambiguous stimulus set

and the column dimersion ( $B$ ) combine in a crossed arrangement. The column dimension $(B)$ and the within-column dimensions ( $C, D$, and $E$ ) form a nested arrangement. This ambiguous set is the basis for the experimental comparison of the crossed and nested classifications. 
The comparison design

We can now describe a comparison experiment which will not confound information load with classification type. Subjects will be asked to recall the attributes of four $3 \times 3$ ambiguous stimulus sets, one after another, after the sets are presented to the subjects in a nested or a crossed temporal order. This design allows the comparison of crossed and nested classifications unconfounded by differing information loads.

The nested order of presentation will focus attention on the columns of the $3 \times 3$ matrix. The subjects will first have presented to them the column dimension ( $B$ in figure 11). Then they will have presented to them the within-column dimensions, $C, D$, and $E$ in figure 11, one column at a time. The row dimension, A in figure 11, will be presented last and by columns. For example, the experimenter will point out that the objects in column one differ in dimension $A$, then that column two differs in dimension $A$, and lastly that column three differs in dimension A. This is the nested order of presentation of the ambiguous stimulus sets.

The crossed order of presentation will focus on the row and column combinations. The column dimension, B in figure 11, will be presented first. The row dimension, $A$ in figure 11, will be presented second. The within-column dimensions, C, D, and $\mathrm{E}$ in figure 11, will be presented last. This is the crossed order of presentation of the ambiguous stimulus set. 
The test of the hypothesis of the temporal induction of structure

It is hypothesized that the two presentation methods will induce the subjects to record different material in long-term memory. It is possible to test this hypothesis. After the last ambiguous set has been recalled, each subject will perform an intervening verbal task. He/she will be asked to count backwards out loud from 100 by threes for one minute. After performing this task, he/she will be asked to recall the last ambiguous set again.

It is hypothesized that each group will recall the attributes of its corresponding classification better than the other group. The crossed group should recall the row attributes better than the nested group, and the nested group should recall the within-column attributes better than the crossed group. The column attributes should be recalled equally well by both groups. Thus the crossed group is hypothesized to have the crossed structure (row and column) of the ambiguous set in long-term memory better than the nested group. Conversely, the nested group is hypothesized to have the nested structure (column and within-column) of the ambiguous set in long-term memory better than the crossed group. These hypotheses will enable us to check the notion that we are indeed looking at the properties of crossed and nested classifications with this experimental procedure.

\section{The error measure}

A measure of error will be taken on the attributes recalled by each subject in each trial. There are three kinds of errors. An 
attribute may not be recalled at all (omission); an attribute may be recalled in the wrong position of the matrix (transposition), and a non-attribute may be recalled (intmusion). Each of these types of errors will count equally towards an overall measure of error. The error measure for each recall will be the simple sum of the individual errors.

The clustering measure

A measure of clustering will also be taken on the attributes recalled by each subject in each trial. Clustering is the tendency for information to be combined into groups, or chunks of related items, during the process of recall. The experimental evidence points to a process of recoding in terms of some meaningful associations or scheme of associations (Klatzky, 1975, pp. 72-73). The meaningful schemes underlying the mixed stimulus sets are the dimensions and their nested or crossed modes of organization. The tendency for clustering in recall will be measured in terms of the five dimensions of the ambiguous set. The measure of clustering used in this experiment is a modified version of the clustering measure first introduced by Cohen, Sakoda, and Bousfield in 1954 (as quoted by Bousfield, Cohen, and Whitmarsh, 1958, p. 42). Since Cohen et al's clustering measure differs in one significant respect from the measure used in this experiment, a detailed derivation is presented.

The specific measure of clustering will be defined in terms of transitions between attributes. As an attribute is recalled, it 
will be written down on a blank $3 \times 3$ matrix by the subject. A number will be placed beside each attribute by the experimenter as it is recalled, indicating its position in the recall sequence, i.e. first, second, third, etc. These recalled attributes will then be plotted on a graph (see figure 12) according to their dimension and recall order.

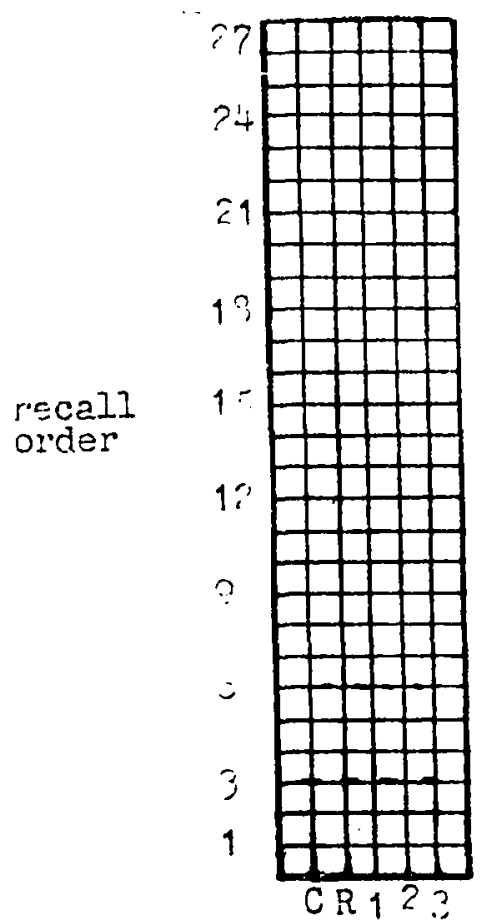

Figure 12. Data grid (where $\mathrm{C}$ is the column dimension, $\mathrm{R}$ is the row dimension, and 1,2, and 3 are the within-column dimensions )

our most direct, intuitive measure of clustering is the number of clusters. A cluster is defined as a series of attributes from the same dimension which are recalled one after another in the recall sequence. A cluster ends when the next attribute in the recall series is from another dimension. Similarly, a cluster begins when 
the immediately preceding attribute is from a dimension different from the immediately succeeding attribute of a pair of attributes. Clusters may vary in length from one to three attributes. It is necessary to include one-attribute clusters in the definition to assure that the range of our clustering measure will always be independent of the number of clusters in a recall series.

The number of transitions between clusters measures clustering as defined above. Any time two clusters are combined into one, the number of transitions between clusters is reduced by one. We will use this idea of between-cluster transitions to define our measure of clustering. Our problem is to propose a measure of clustering so that as the number of transitions between clusters decreases, the measure of clustering increases.

our clustering measure is defined in terms of the transitions between consecutively recalled attributes. Let "N" be the number of attributes recalled by one subject in one recall trial. Thus $0 \leq N \leq 27$. Let " $T$ " be defined as the number of transitions for that same trial. Thus, $T=N-1$, when $1 \leq N$; so that $T=0$, when $\mathrm{N}=1$. $\mathrm{T}$ is also defined as zero when $\mathrm{N}=0$. Call a transition a "within-dimension transition" if the two consecutively recalled attributes are from the same dimension. Call a transition an "acrossdimension transition" if the two consecutively recalled attributes are from different dimensions. These across-dimension transitions are the same as the transitions between clusters. On the graph in figure 12 (see also figure 14 and figure 16), the across-dimension transitions appear as diagonal lines; the within-dimension transitions 
as vertical lines. Let " $D$ " be defined as the number of across-dimension transitions in a recall trial. Thus $D \leq T . D$ is also thereby the number of diagonal lines on the data graph of the recall trial. These transitions allow us to define a clustering measure.

The clustering measure is defined using the number of transitions ( $T$ ), the number of diagonals (D), and the number of stimulus dimensions from one recall trial. Let "DM" be defined as the number of stimulus dimensions whose attributes were recalled during the recall trial. Thus, $0 \leq D M \leq 5$. Our clustering measure is defined as follows:

$$
C=\frac{T-D}{T-(D M-1)}
$$

when $0<D M<N$, and as $C=0$ when $0 \leq D M=N$. This is our measure of the clustering present in a recall trial.

$C$ has several useful properties. When clustering is minimum, $C$ has its minimum value of 0 for all recall sequences. As clustering increases, the number of diagonals decreases and $C$ increases. Our problem is to show that as the number of diagonals drops, the value of $\mathrm{C}$ increases but stays between 0 and 1 inclusive. These properties will be proved below.

First we need to define the conditions of maximum and minimum clustering. Clustering is at a maximum when all the attributes of one dimension are recalled consecutively in one cluster, then all the attributes of another dimension are recalled one after another in one cluster, and so on until recall is ended. Each dimension's attributes are recalled separately in a cluster with no mixing with any other dimension's attributes. When clustering is maximum, the 
number of clusters equals the number of dimensions recalled. The transitions between these clusters will be one less than the number of clusters. Thus when clustering is maximum, $D=D M-1$. Clustering is at a minimum when every attribute is immediately preceded in the recall sequence and immediately followed by an attribute of a different dimension. In this case all transitions are diagonals. Thus when clustering is minimum, $T=D$. We have the conditions which define the maximum and minimum clustering.

We can use the maximum and minimum conditions to show that $\mathrm{C}$ is also respectively maximum and minimum. For the moment, consider the cases where $0<\mathrm{DM}<\mathrm{N}$. These cases divide themselves into five groups. Each group is defined by the number of dimensions involved in the recall sequence, i.e. $\mathrm{DM}=5, \mathrm{DM}=4$, etc. When clustering is a maximum, $D=D M-1$. Thus

$$
C(\max )=\frac{T-(D M-1)}{T-(D M-1)}=1.00 .
$$

When clustering is a minimum, $T=D$. Thus

$$
C(\min )=\frac{(D-D)}{T-(D M-1)}=0 \text {. }
$$

When the attributes of only one dimension are recalled, we can have only one cluster. In this case, $D M=1$ and $D=0$. Thus

$$
C=\frac{T-0}{T-(1-1)}=\frac{T}{T}=C(\max ),
$$

which is what we needed. For the cases considered, we have shown that $C=1$ when clustering is maximum and $C=0$ when clustering is minimum. 
We can also show that $\mathrm{C}$ assumes only those values between 1 and 0 , and furthermore that as clustering increases, $C$ correspondingly increases. Consider as above, those cases where $0<\mathrm{DM}<\mathrm{N}$. As above, these cases divide themselves into five groups, i.e. $D M=5$, $D M=4$, etc. Within any group DM is a constant. Within any one group there may be a range of possibilities for $\mathrm{T}$ from a maximum of 26 to DM. Consider any fixed $\mathrm{T}$ in any group. This sets a bound for D. In particular, $T$ sets an upper bound on $D$ because $D$ is defined as a subset of $T$. Thus $D(\max )=T$. The minimum value of $D$ is defined by the number of dimensions in the group, and is simply $D=D M-1$. Thus for any given group and any given $T, D$ ranges between $D(\max )=T$ and $D(\min )=D M-1$. However, these bounds on $D$ are precisely the conditions for minimum and maximum clustering, respectively, as just shown above. Thus we have shown that the value of $\mathrm{C}$ ranges from 0 to 1 as clustering varies from minimum to maximum respectively for the conditions considered.

We can show that as clustering increases, the value of $\mathrm{C}$ also increases. As clustering increases, the value of $D$ decreases. If we can show that the value of $C(D-1)-C(D)$ is always positive for the cases considered, then we can show that the value of $C$ increases as clustering increases. Let $D M$ and $T$ be arbitrarily fixed, i.e. we are considering one of the five groups defined above. In this case, we have the following derivation; 


$$
\begin{aligned}
C(D-1)-C(D) & =\frac{T-(D-1)}{T-(D M-1)}-\frac{T-D}{T-(D M-1)} \\
& =\frac{T-D+1-T+D}{T-(D M-1)} \\
& =\frac{1}{T-(D M-1)} .
\end{aligned}
$$

We have limited ourselves to the cases where $0<D M<N$. At the minimum $N, N=D M+1$, which implies $T=N-1=D M$. For all other cases $\mathrm{T}>\mathrm{DM}$. So that $0<\mathrm{T}-(\mathrm{DM}-1)$ for all $0<\mathrm{DM}<\mathrm{N}$. Thus, $0<C(D-1)-C(D)$ for $D M$ and $T$ arbitrarily fixed. We have show 1 that as clustering increases, the value of $\mathrm{C}$ increases, and has a maximum of 1 and a minimum of 0 for maximum and minimum clustering when $0<\mathrm{DM}<\mathrm{N}$.

Let us consider the cases where $0 \leq \mathrm{DM}=\mathrm{N}$. These are the cases where a subject recalls only one attribute from each of several dimensions, or one attribute from only one dimension, or nothing at a11. If he recalls only one attribute from each of several dimensions, he has only zero order clusters and hence no clustering. In this case, $T=N-1=D M-1$. If we substitute this value for $T$ into our definition of $\mathrm{C}$, we find ourselves with a denominator of zero, and an indeterminate value for $C$. For these reasons, when a. subject recalls only one attribute from each of several dimensions, one attribute from one dimension, or nothing, $\mathrm{c}=0$ by definition. These cases complete the proof that the value of $\mathrm{C}$ corresponds to the amount of clustering in a recall trial. This measure of clustering will be taken on each recall trial. 
The measure of clustering, $C$, differs in one important respect from the repetition ratio, $\mathrm{RR}$, introduced by Cohen, Sakoda, and Bousfield in 1954 (as quoted by Bousfield, Cohen, and Whitmarsh, 1958, p. 42) aric defended by Frender and Doubilet in 1974 as the best available measure of clustering. When clustering is maximum, $\mathrm{RR}$ has a variable upper limit dependent on the number of items recalled. In contrast when clustering is maximum, $C$ has a fixed upper limit which is independent both of the number of items recalled and the number of categories recalled. This fixed independent upper limit of $\mathrm{C}$ allows us to compare clustering on the same trial across subjects when subjects recall different numbers of categories and make different numbers of errors when attempting to recall the same material.

$R R$ is defined as $R R=r /(n-1)$, where $n$ is the number of items recalled and $r$ is the number of repetitions. "A repetition is defined as the temporarily contiguous recall of two items from the same category." (Frender \& Doubilet, 1974, p. 64) The number of repetitions is the same as the number of within-dimension transitions so that $r=T-D$. The numerators of $C$ and $R R$ are the same. The denominator of $\mathrm{C}$ differs from that of $\mathrm{RR}$ by a difference of (DM - 1), since $T=n-1$. This difference in their denominators gives $C$ a fixed upper limit and $R R$ a variable upper limit. As an example of this difference, consider two subjects who recall different numbers of items in different categories on the same trial, but who use maximum clustering in their recall sequences. Subject one recalls 10 items in 3 categories. In this case, $n=10$, 
$\mathrm{T}=9, \mathrm{DM}=3, \mathrm{D}$ (maximum clustering $)=\mathrm{DM}-1=2$, and $\mathrm{r}=\mathrm{T}-\mathrm{D}=7$. Thus $R R=r /(n-1)=7 / 9=.78$ whereas $C=(T-D) /(T-(D M-1))=$ $=1.00$. Subject two recalls 27 items in 4 categories. In this case $R R=.88$ whereas $C=1.00$. In these two cases when clustering is at a maximum, $R R$ gives two different values where $C$ gives the same value. For our purposes $\mathrm{C}$ will be used to measure clustering.

\section{HYPOTHESES}

The error hypotheses

It is hypothesized that more errors will be made by the crossed group than the nested group. This prediction is made on the basis of the experimental evidence presented earlier. The "canary" experiment (Collins \& Quillian, 1969) suggests that nested structures are more commonly used than crossed structures. Subjects should have had more practice using the nested structure, even though the crossed structure can be shown to be more efficient. Subjects should be more efficient using a less efficient structure with which they are familiar, than they would be using a more efficient structure with which they are unfamiliar. The nested structure should be easier to learn on that basis.

The clustering hypothesis

It is hypothesized that there will be a greater number of clusters in the recall of the nested group than in the recall of the crossed group. The nested group has the column aspect of the stimuli emphasized. The nested group should tend to focus on 
recalling the attributes by columns. The row dimension is presented to the crossed group so that it breaks up the columns. The crossed group should focus more on recalling the stimulus dimensions than on breaking the dimensions up by columns. It is also hypothesized that this clustering pattern will persist in the recall of the fourth set material after the intervening task.

\section{The learning curve hypothesis}

Each experimental group will be presented with four mixed stimulus sets. It is hypothesized that the error and clustering measures will exhibit learning curves. As the number of recall trials increases, the two groups should diverge on the clustering measure and converge on the error measure.

The subject expectation effect hypothesis

The results may be affected by bias on the part of subjects either to conform to or violate the subjects' perception of what the experimenter expects to find (Rosenthal, 1966). Call this effect the subject expectation effect (SEE). Within the constraints of this experimental design, it is possible to detect the presence or absence of any SEE. If a SEE is present, the subjects will not only have to remember the stimulus material, but will also have to remember information about the perceived bias of the experimenter and to perform operations on the recalled material. If a SEE is present, then this extra load should degrade performance. In order to test for a $\mathrm{SEE}$, some way is needed to measure the 
subject's perception of what the subject thinks the experimenter wants the subject to find. This cannot be done directly. Instead, one possible perception of the experimenter's orientation will be proposed by inspection and measures derived for that perception. Subjects are presented the dimensions in a particular order. For the nested group, the order is column, within-column, and row dimension. For the crossed group, the order is column, row, and withincolumn dimension. If a SEE is present, it is hypothesized that subjects will perceive this order as the experimenter's bias. If subjects code the stimulus material into memory in the order of presentation and are prone to a SEE which leads them to violate their perceptions of the experimenter's bias, they will rearrange the material before writing it down. If, on the other hand, subjects code the stimulus material into memory in an order different from the order of presentation, and are prone to a SEE which leads them to conform to their perception of the experimenter's order, they will also rearrange the material before written recall.

The concept of rearrangement can be operationalized by measuring changes in the order of recall of the stimulus dimensions. For an example, suppose subject $J$ recalls a series of row attributes, then a series of column attributes, and finally a series of withincolumn attributes. Also suppose subject $K$, in the same treatment group, may use the opposite order, i.e. a series of within-column attributes, then a series of column attributes, and lastly a series of row attributes. The important question is whether the rearrangement by subject $\mathrm{J}$ or $\mathrm{K}$ increases errors, decreases errors, or has no 
effect. For a second example, suppose subject $F$ recalls several row attributes, then several column attributes. Also suppose subject $G$ recalls only 1 row attribute and then 1 column attribute. A second important question is whether the recall of attributes of one dimension in a long or short series increases, decreases, or has no effect on errors. The rearrangement measure needs to be operationalized to measure dimensional recall order and length of recall between dimensions.

Three measures of this rearrangement are proposed. Let "Col" be defined as the first appearance of the column dimension attributes in a recall trial. Let " $R$ " be defined as the first appearance of the row dimension attributes in a recall trial. Let "WC" be defined as the first appearance of the within-column dimension attributes in a recall trial. Let " $R_{i}$ " be defined as the $i^{\text {th }}$ measure of rearrange ment. $R_{1}=R-$ Col. $R_{2}=W C-$ Col. $R_{3}=W C-R$.

These $R_{i}$ 's have the two properties described above. Dimensional recall order is measured by the sign of each $R_{i}$. If the column attributes are recalled before the row attributes, then $R_{1}$ will be positive. If the column attributes are recalled after row attributes, then $R_{1}$ will be negative. Recall length between dimensions is also measured by each $R_{i}$. As the number of attributes between the first appearance of any two dimensions increases, then the absolute magnitude of the corresponding $R_{i}$ will also increase. These $R_{i}$ have the properties needed to operationalize the concept of rearrangement.

The measures of rearrangement can detect the presence of SEEs by correlations with recall errors. If a SEE is present, then the 
subject must go through extra steps between memorization and written recall. When asked to recall the stimulus material, the subject will first recall the material in the form in wich the subject memorized it. The subject will then rearrange the material before writing it down. This rearrangement will impose an extra memory load that non-rearranging subjects won't have. Thus rearranging subjects should show more errors than non-rearranging subjects. Therefore, as the degree of rearrangement changes, the amount of error should also change if a SEE is present.

The presence of a SEE of the type described will reveal itself as a significant correlation between some of the rearrangement measures and the error measure. The correlations will be calculated separately for the crossed and nested groups, as one group may have a SEE and the other not. It is hypothesized that no strong, significant correlations will be found.

Summary

Two subject groups will be presented with the same four ambiguous stimulus sets. One group will be presented with the sets in a crossed order, the other with the sets in a nested order. Each group will be asked to recall each set immediately after presentation. After the fourth set is recalled, the subjects will be asked to count backwards, out loud, by three, from 100, for one minute, then to recall the material of the fourth set again. Five measures will be taken on the recalled material, a measure of errors, a measure of clustering, and three measures of rearrangement. 
It is hypothesized (1) that in short-term recall the nested group will make fewer errors than the crossed croup; (2) that the nested group will show less clustering than the crossed group in short-term recall; (3) that both error and clustering measures will exhibit learning curves; (4) that the structure of each group will be induced in long-term memory, and (5) that no subject expectation effect will be detected. 


\section{CHAPTER II}

\section{METHOD}

SUBJECTS

Twenty four undergraduate volunteers from Portland State University psychology courses were randomly assigned to the two experimental groups.

\section{SETTING}

The experiment was conducted in a quiet room, free from noise, distraction, and extraneous personnel, and equipped with a table and two chairs.

\section{MATERIALS}

\section{Stimulus sets}

The experiment used 20 ambiguous stimulus sets. The stimulus sets are divided into four groups on the basis of their content, yielding five sets in each content group. The contents are pictures of bottles, houses, mountains, and letter "A"s. Each of the pictures in a set varies along five dimensions. For example, the dimensions of the pictures of the mountains are the width of the peak, the number of peaks, the height of the peaks, the number of clouds, and the amount of snow. Each dimension may appear as any of the five 
ambiguous stimulus dimensions, i.e. as a row, column, etc. The pictures of the other three content sets also have five dimensions, which are listed in Appendix F.

The stimulus sets are represented graphically in two different ways to emphasize the crossed or nested aspects of the sets. The sets shown to the nested group have thicker lines dividing the columns than dividing the rows. These lines emphasize the column aspect of the stimulus set. The sets shown to the crossed group have lines of equal thickness dividing the rows and the columns. These lines emphasize the crossed structure of the stimulus set. The two physical formats are presented in Appendix D.

TABLE I

THE FIVE MOUNTAIN STIMULUS SETS ${ }^{2}$

\begin{tabular}{|c|c|c|c|c|c|c|c|}
\hline & \multicolumn{5}{|c|}{ Content Mode Rotation } \\
\hline & & & 1 & 2 & 3 & 4 & 5 \\
\hline \multirow{5}{*}{ Dimension } & \multicolumn{2}{|l|}{ Row } & W & $\mathrm{s}$ & C & $\mathrm{H}$ & $P$ \\
\hline & \multicolumn{2}{|l|}{ Column } & $\mathrm{P}$ & $\mathrm{W}$ & S & C & $\mathrm{H}$ \\
\hline & \multirow[t]{3}{*}{$\begin{array}{l}\text { Within- } \\
\text { Column }\end{array}$} & 1st & $H$ & $P$ & W & $S$ & C \\
\hline & & 2nd & C & $\mathrm{H}$ & $P$ & W & $\mathrm{S}$ \\
\hline & & $3 r a$ & $S$ & C & $\mathrm{H}$ & $P$ & W \\
\hline
\end{tabular}

a. W refers to the peak width, $P$ to the number of peaks, $H$ to the height of the peaks, $C$ to the number of clouds, and $S$ to the amount of snow

Recall sheets

Subjects have been asked to give a written recall after exposure 
to an ambiguous set. The two groups were given slightly different recall sheets. Each sheet has nine cells arranged in a $3 \times 3$ matrix. Each cell has three dotted lines where the subjects may write the attributes which describe the picture in the corresponding cell of the stimulus set. The physical format of the recall sheets for each group is the same as the physical format of the stimulus sets for that group. The nested recall sheets use heavy lines to divide its columns. The crossed recall sheets use equal lines to divide the rows and columns. Examples of the two recall sheets are given in Appendix C.

\section{Instructions}

Each subject was given one page of instructions to read. These instructions describe in a general way what the subjects would be asked to do. The instructions are in Appendix A.

\section{Practice stimulus set}

Each subject was given one practice stimulus set to recall before exposure to and recall of the four main sets. This practice set contains pictures of lamps with five stimulus dimensions arranged in an ambiguous stimulus set. Similar to the stimulus sets, each of the stimulus dimensions is rotated through the five dimensional positions. There are five practice sets. The rotations are coded in Appendix $\mathrm{E}$, which also contains the five practice sets.

$$
\text { PROCEDURE }
$$

For any subject, there were four experimental steps. First, 
the subject read the instructions. Second, the practice set was presented to the subject who wrote down his recall with the practice set still in view. The experimenter told the subject of any procedural errors during the practice trial. Third, the subject was successively presented with four crossed or nested stimulus sets in a crossed or nested presentation, respectively, which the subject then attempted to recall immediately after each presentation. Fourth, the subject performed the intervening verbal task, and then attempted to recall the fourth stimulus set. After these steps, the subjects were asked to describe how they recalled the material, and, in particular, whether or not they used visual images.

Each experimental group had the dimensions of each stimulus set presented to them in the special order for that group. Each set was presented twice before any recall was attempted. After the second presentation, the stimulus set was removed, and the subject was given a blank recall sheet. The protocols for the crossed and nested presentation orders are listed in Appendix B.

During the presentation of each dimension, the subjects were asked to give their own labels for each of the attributes. If they were at a loss to provide a label, the experimenter would suggest labels they could use. If a label was used for two attributes, the experimenter pointed this out to the subject, and asked for a second label. The subjects were using their own labels as much as possible.

DESIGN

The experiment, as a whole, is designed to measure the effects 
of crossed and nested presentations across trials. The experiment is not concerned with the effects of specific contents or content modes. The content effects are minimized by a Latin square design as illustrated in table II. The effects of stimulus dimension are only partially minimized because fewer subjects are used than there are stimulus dimensions in each of the four stimulus sets.

\section{TABLE II}

$$
\begin{aligned}
& \text { ASSIGNMENT TO SUBJECTS OF } \\
& \text { STIMULUS SETS BY CONTENT } \\
& \text { MODE, TRIAL ORDER, } \\
& \text { AND SET CONTENT }
\end{aligned}
$$

\begin{tabular}{c|c|c|c|c|c}
\hline $\begin{array}{c}\text { Subject } \\
\text { Number }\end{array}$ & $\begin{array}{l}\text { Content } \\
\text { Mode } \\
\text { Rotation }\end{array}$ & \multicolumn{4}{|c|}{ Trial } \\
\cline { 2 - 6 } & 1 & 2 & 3 & 4 \\
\hline 2 & 2 & B & A & M & H \\
3 & 3 & & & & \\
4 & 4 & & & & \\
5 & 5 & $\mathrm{H}$ & B & A & M \\
6 & 1 & & & & \\
7 & 2 & & & & \\
8 & 3 & $M$ & H & B & A \\
9 & 4 & & & & \\
10 & 5 & & & & \\
\cline { 2 - 6 } 11 & 1 & A & M & H & B \\
12 & 2 & & & & \\
\hline
\end{tabular}

a. B refers to bottles, A to the letter A, $M$ for mountains, and $H$ to houses 
DATA COTIECTION

Two kinds of raw data were collected; the items recalled and the order in which they were recalled. As the subject wrote the recalled material on the recall sheet, the experimenter marked the order of recall beside each recalled attribute. This enumeration was then coded onto the data grid together with the item data (see figure 12). The items were recorded as correct recalls or as errors of various types. If an attribute was correctly recalled, a filled dot was marked at the intersection of the appropriate dimension and recall order. If an attribute was recalled in the wrong cell, an empty dot was marked at the intersection of the dimension of the attribute and the recall order. If the dimension to which a recalled attribute belongs was unclear, the experimenter sought clarification from the subject in as non-suggestive a manner as possible during the experiment.

\section{DATA CODTNG EXAMPLES}

Two examples of data coding will be presented. In each case, an imaginary protocol will be presented, coded, and transcribed onto a data grid. The first protocol represents a case where clustering is minimal (see figure 13 and figure 14). The second protocol represents a case where clustering is maximal (see figures 15 and 16). Both protocols represent recall attempts of the first mountain set (see appendix F) as performed by a subject in the nested group. The process of data coding is illustrated below. Each recall 
of the protocol contains three attribute labels. Beside each label is a number which represents the position of that attribute in the recall sequence of the subject. The first word written down by the subject has a " 1 " placed beside it. The second word has a " 2 " placed beside it, etc., all the way up to the last word written down. Also beside each attribute will be coded the dimension to which the attribute belongs. "C" will be placed beside the column attributes and "R" beside row attributes. The within-column attributes will not have a code marked beside them if they are correct. Whenever an attribute is incorrect, an "X" will be marked beside it. If the attribute is in the wrong dimensional position (for example, an attribute from the first within-column dimension may be recalled in the third column), the dimension to which the attribute belongs for that stimulus set will be coded in parentheses beside the $X$. The sequence numbers, dimension codes, and error marks will then be transferred to the data grid. 


\begin{tabular}{|c|c|c|c|c|}
\hline $\begin{array}{l}\text { tall } \\
\text { narrow } \\
1 \text { peak }\end{array}$ & $\begin{array}{ll}1 & \\
2 & R \\
3 & C\end{array}$ & $\begin{array}{l}1 \text { cloud } 10 \\
\text { narrow } 11 \mathrm{R} \\
2 \text { peaks } 12 \mathrm{C}\end{array}$ & $\begin{array}{l}\text { tall } \\
\text { narrow } \\
3 \text { peaks }\end{array}$ & $\begin{array}{l}19 \mathrm{X}(1) \\
20 \mathrm{R} \\
21 \mathrm{C}\end{array}$ \\
\hline $\begin{array}{l}\text { medium } \\
\text { height }\end{array}$ & 4 & 2 clouds 13 & $\begin{array}{l}\text { medium } \\
\text { height }\end{array}$ & $22 \times(1)$ \\
\hline $\begin{array}{l}\text { medium } \\
\text { width }\end{array}$ & $5 \mathrm{R}$ & $\begin{array}{l}\text { medium } \\
\text { width }\end{array}$ & $\begin{array}{l}\text { medium } \\
\text { width }\end{array}$ & $23 \mathrm{R}$ \\
\hline 1 peak & $6 \mathrm{c}$ & 2 peaks $15 \mathrm{C}$ & 3 peaks & $24 \mathrm{C}$ \\
\hline short & 7 & 3 clouds 16 & short & $25 \times(1)$ \\
\hline wide & $8 \mathrm{R}$ & wide & wide & $26 \mathrm{R}$ \\
\hline 1 peak & $9 \mathrm{C}$ & 2 peaks $18 \mathrm{C}$ & 3 peaks & $22 \mathrm{C}$ \\
\hline
\end{tabular}

Figure 13. An example of minimum clustering (in the recall protocol of a subject in the nested group who recalled the first mountain set).



Figure 14. Minimum clustering on a data grid. (Transcribed from the protocol in figure 13) 


\begin{tabular}{|c|c|c|c|c|c|}
\hline $\operatorname{tall}$ & 19 & 1 cloud & 22 & snow & 25 \\
\hline narrow & $10 \mathrm{R}$ & namrow & $11 \mathrm{R}$ & narrow & 12 \\
\hline 1 peak & $1 \mathrm{C}$ & 2 peaks & $4 \mathrm{C}$ & 3 peaks & 7 \\
\hline $\begin{array}{l}\text { medium } \\
\text { height }\end{array}$ & 20 & 2 clouds & 23 & snow & \\
\hline $\begin{array}{l}\text { medium } \\
\text { width }\end{array}$ & $13 \mathrm{R}$ & $\begin{array}{l}\text { medium } \\
\text { width }\end{array}$ & $14 \mathrm{R}$ & $\begin{array}{l}\text { medium } \\
\text { width }\end{array}$ & 15 \\
\hline short & 21 & 3 clouds & 24 & snow & 27 \\
\hline wide & $16 R$ & wide & $17 \mathrm{R}$ & wide & 18 \\
\hline 1 peak & $3 c$ & 2 peaks & $6 \mathrm{c}$ & 3 peaks & 9 \\
\hline
\end{tabular}

Figure 15. An example of maximum clustering (in the recall protocol of a subject in the nested group who recalled the first mountain set).

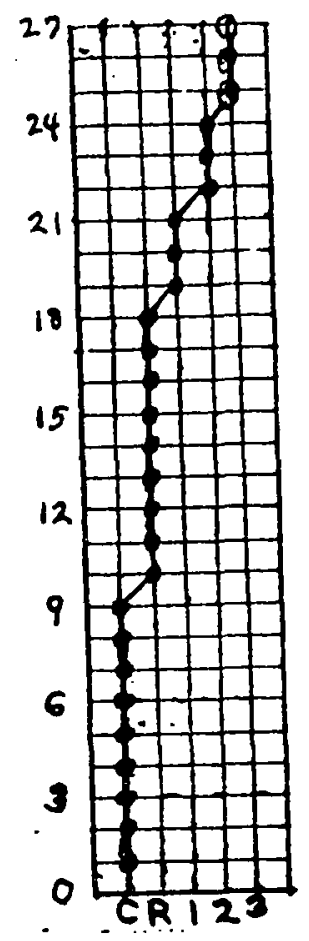

Figure 16. Maximum clustering on a data grid. (Transcribed form the protocol in figure 15) 
CHAPTER III

RESULTS

The experiment produced a few significant differences between the two treatment groups. In particular, there were two results of note. The correlations of the subject expectation effect hypothesis suggest that rearrangement affects errors (see table VIII). Posthoc analyses were performed to explore the implications of these correlations. The t-tests of dimension specific errors of the induction of structure hypothesis suggest that the two classifications are treated differently in long-term memory (see table VIII). Post-hoc analyses were performed to complete the analyses of short and long-term memory errors. Table III summarizes the analyses, both those performed to test the experimental hypotheses and those that were performed post-hoc. 
TABLE III

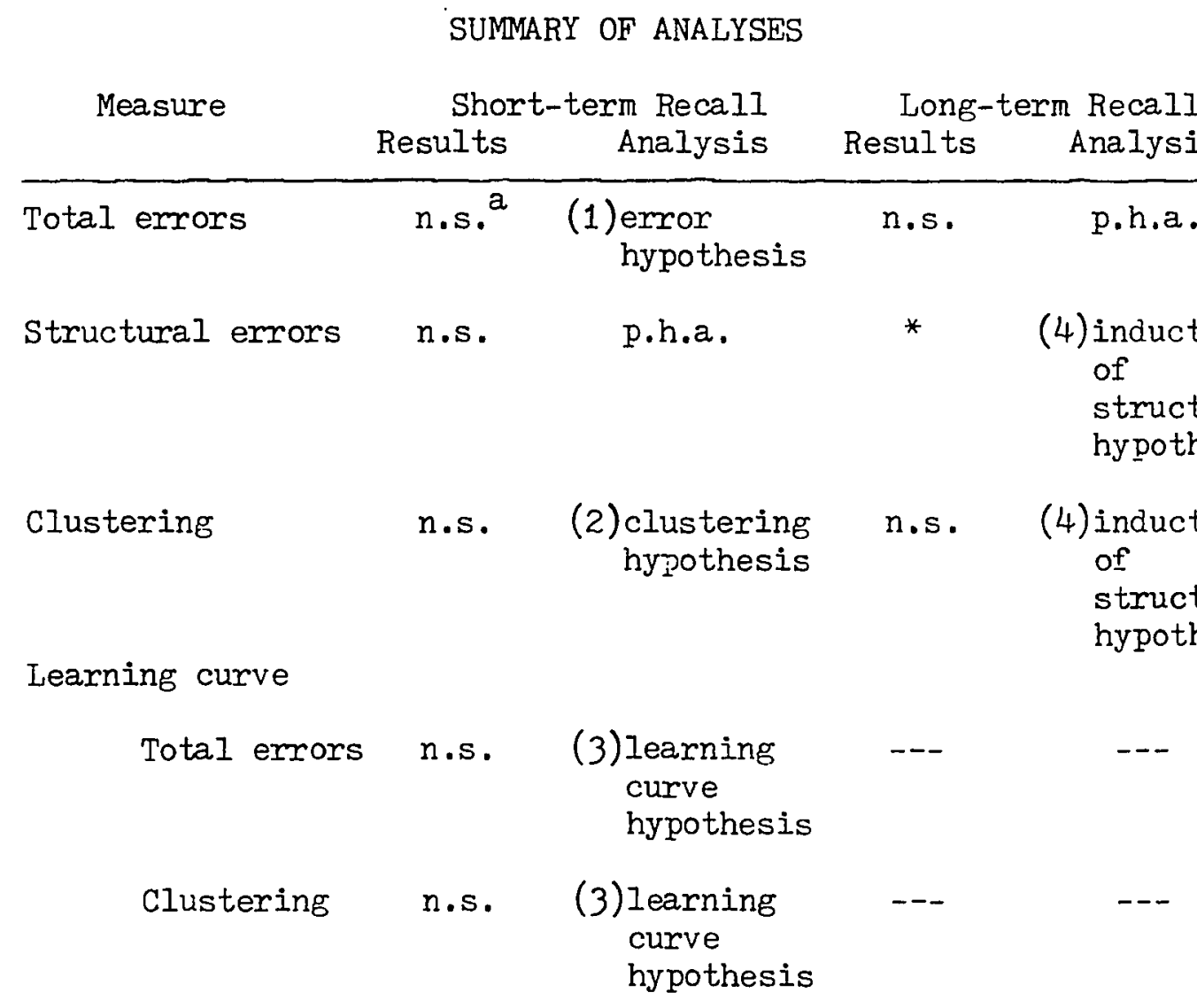

Rearrangement correlations

\begin{tabular}{llll} 
Rearrangement & $*$ & $\begin{array}{c}\text { (5) subject } \\
\text { expectation } \\
\text { effect } \\
\text { hypothesis }\end{array}$ & n.s. \\
$\begin{array}{l}\text { Absolute } \\
\text { rearrangement }\end{array}$ & p.h.a. & n.s. & p.h.a. \\
\hline
\end{tabular}

a. not significant

b. post-hoc analysis

* significant differences, $\mathrm{p}<.05$ 


\section{RESULTS OF EXPERIMENTAI HYPOTHESES}

\section{(1) Error hypothesis}

The error data from the first four trials were subjected to a $2 \times 4$ (treatment mode $\mathrm{x}$ trials) analysis of variance (ANOVA) with repeated measures on the treatment mode variable using conservative degrees of freedom and post-hoc comparisons on the trials factor (Winer, 1971, pp. 514-539). There were no significant differences at the .05 level in the error dependent variable between the two treatment groups $(F(1,22)=.08$, see table IV).

\section{TABIE IV}

ANOVA FOR ERROR DATA,

USING CONSERVATTVE

DEGREES OF FREEDOM

\begin{tabular}{|c|c|c|c|c|}
\hline SOURCE & SS & $\mathrm{DF}$ & MS & $F$ \\
\hline Between Subjects & 485.16 & 23 & & \\
\hline Treatments & 1.76 & 1 & 1.76 & $\begin{array}{c}\mathrm{F}_{1,22}=.08 \\
\text { n.s. }\end{array}$ \\
\hline $\begin{array}{l}\text { Subjects within } \\
\text { groups } \\
--------\end{array}$ & $\begin{array}{l}483.40 \\
----\end{array}$ & $\begin{array}{r}22 \\
--\end{array}$ & $\begin{array}{l}21.97 \\
---\end{array}$ & ------ \\
\hline Within Subjects & 557.21 & 72 & & \\
\hline Trials & 34.12 & 3 & 11.37 & $\begin{array}{c}F_{1,22}=1.47 \\
\text { n.s. }\end{array}$ \\
\hline Trials $x$ Treatment & 12.28 & 3 & 4.09 & $\begin{array}{c}\mathrm{F}_{1,22}=.53 \\
\text { n.s. }\end{array}$ \\
\hline $\begin{array}{c}\text { Trials } x \text { Subjects } \\
\text { within groups }\end{array}$ & 510.85 & 66 & 7.74 & \\
\hline
\end{tabular}




\section{(2) Clustering hypothesis}

The clustering data for the first four trials were transformed by an arcsin transformation to ensure equal variances (Winer, 1971, pp. 399-400). The transformed clustering data were then subjected to the same ANOVA as the error data. There were no significant differences at the .05 level in the clustering dependent variable between the two treatment groups $(F(1,22)=2.70$, see table $V)$.

\section{TABLE V}

\section{ANOVA FOR THE ARCSIN TRANSFORM OF THE CLUSTERING DATA, USING CONSERVATIVE DEGREES OF FREEDOM}

\begin{tabular}{|c|c|c|c|c|}
\hline SOURCE & SS & $D F$ & MS & $\mathrm{F}$ \\
\hline Between Subjects & 77.32 & 23 & & \\
\hline Treatments & 8.46 & 1 & 8.46 & $\begin{array}{c}F_{1,22}=2 \cdot 70 \\
\text { n.s. }\end{array}$ \\
\hline $\begin{array}{l}\text { Subjects within } \\
\text { groups }\end{array}$ & $\begin{array}{r}68.86 \\
-----\end{array}$ & 22 & 3.13 & $-\ldots-\ldots$ \\
\hline Within Subjects & 32.98 & 72 & & \\
\hline Trials & 3.96 & 3 & 1.32 & $\begin{array}{c}\mathrm{F}_{1,22}=3.07 \\
\mathrm{p}<.10\end{array}$ \\
\hline Trials $x$ Treatment & .88 & 3 & .29 & $\begin{array}{c}\mathrm{F}_{1,22}=.67 \\
\text { n.s. }\end{array}$ \\
\hline $\begin{array}{l}\text { Trials } \times \text { Subjects } \\
\text { within groups }\end{array}$ & 28.14 & 66 & .43 & \\
\hline
\end{tabular}


(3) Learning curve hypothesis

Errors. There were no significant differences at the .05

level in the error dependent variable across the trials $(F(1,22)=1.47$, see table IV). There were no significant interactions at the .05 level between the error dependent variable and the trials factor $(F(1,22)=.53$, see table IV $)$.

Clustering. There were differences at the .10 level in the clustering dependent variable across the first four trials $(F(1,22)=3.07$, see table $V)$. A test on the trial means using the Newman-Keuls procedure (Winer, 1971, pp. 528-529) was performed. This test showed that the average clustering on the first trial was significantly different at the .05 level from the average clustering on the second, third, and fourth trial (see table VI). The NewmanKeuls procedure was performed on the arcsin transformation of the clustering measure. After transformation back, the mean clustering on the first trial was .61 , on the second .76 , on the third .82 , and on the fourth .83. There was no significant interaction effect at the .05 level between the clustering dependent variable and the trials factor $(F(1,22)=.67$, see table $V)$. 
TABLE VI

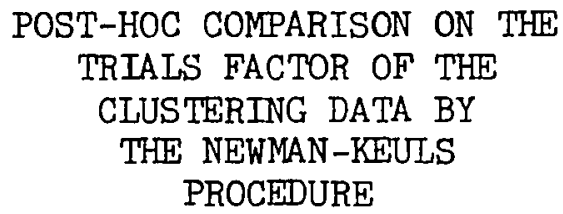

i. $\begin{array}{lllll}\text { trials } & t_{1} & t_{2} & t_{3} & t_{4} \\ \begin{array}{l}\text { ordered } \\ \text { means }\end{array} & 1.79 & 2.12 & 2.27 & 2.30\end{array}$

ii.

\begin{tabular}{ccccccc} 
& $t_{2}$ & $t_{3}$ & $t_{4}$ & $r$ & $\left(S_{T}\right)$ & $(q .95$ \\
\hline$t_{1}$ & 0.33 & 0.48 & 0.51 & 4 & 0.2502 \\
\hline$t_{2}$ & & 0.15 & 0.18 & 3 & 0.2275 \\
\hline$t_{3}$ & & & 0.03 & 2 & 0.1893 \\
\hline
\end{tabular}

iii. $S_{T}=\left\{\frac{M S_{T} \times S W G}{96}\right\}^{\frac{1}{2}}=0.0669, d f=66$

\begin{tabular}{|c|c|c|c|}
\hline iv. & 2 & 3 & 4 \\
\hline $\mathrm{q} .95^{(r, 66)}$ & 2.83 & 3.40 & .74 \\
\hline
\end{tabular}

v. significant differences (*)

$\begin{array}{lccc} & t_{2} & t_{4} & t_{3} \\ t_{1} & * & * & * \\ t_{2} & & - & - \\ t_{4} & & & -\end{array}$


(4) Induction of structure hypethesis

Errors. The error data from the recall trial aftex the intervening task (fifth trial) were subjected to $\underline{t}$ tests (Bruning \& Kintz, 1968, pp. 9-12). There was a significant difference at the .05 level between the two treatment groups in the within-column error dependent variable (see table VII). There was no significant difference at the .05 level between the two treatment groups in the row error dependent variable. There was no significant difference at the .05 level between the two treatment groups in the column error dependent variable.

TABLE VII

LONG-TERM RECALL ERROR DATA, MEANS, STANDARD DEVIATIONS, AND T-TEST

VALUES FOR DIFFERENCES

\begin{tabular}{lcccccc}
\multirow{2}{*}{ dimension } & \multicolumn{4}{c}{ group } & $\underline{t}^{\mathrm{a}}$ \\
& crossed & nested & \\
\cline { 2 - 6 } & mean & s.d. & mean & s.d. \\
\hline column & 0.92 & 2.61 & 0 & 0 & 1.61 \\
row & 1.75 & 2.70 & 0.83 & 1.53 & 1.02 \\
within-column & 1.90 & 3.45 & 0 & 0 & $1.89 *$ \\
\hline
\end{tabular}

a. $\mathrm{df}=22$, one-tailed test

* $\mathrm{p}<.05$

Clustering. The clustering data from the recall trial after the intervening task (fifth trial) were subjected to a $t$ test (Bruning \& Kintz, 1968, pp. 9-12). The difference between the two groups was not significant at the .05 level. For the crossed group, the mean clustering score was .76 with a standard deviation of .51 . 
For the nested group, the mean clustering score was .70 with a standard deviation of .38 .

\section{(5) Subject expectation effect hypothesis}

The error data and the rearrangement data of the first four trials were subjected to Pearson product-moment correlations and significance tests (Bmuning \& Kintz, 1968, pp. 152-155). Of the six Pearson product-moment correlations, two were significant, one at the .02 level and one at the .01 level (see table VIII). In the crossed group, the correlation between the second rearrangement variable and the error variable was $-.35(z=-2.40)$. Also in the crossed group, the correlation between the third rearrangement variable and the error variable was $-.31(z=-2.13)$.

TABLE VIII

PEARSON PRODUCT-MONENT' CORRELATIONS BETWEEN $R_{i}$ AND ERRORS

\begin{tabular}{|c|c|c|c|}
\hline group & $\mathrm{R}_{i}$ & $r$ & $z$ \\
\hline & 1 & -.14 & -.96 \\
\hline \multirow[t]{2}{*}{ Crossed } & 2 & -.35 & $-2.40 * *$ \\
\hline & $\frac{3}{1}$ & $\begin{array}{l}-.31 \\
-.05\end{array}$ & $\begin{array}{c}-2.13 * \\
-.31\end{array}$ \\
\hline \multirow[t]{2}{*}{ Nested } & 2 & -.17 & -1.17 \\
\hline & 3 & -.02 & -.17 \\
\hline
\end{tabular}

* $\mathrm{p}<.02$

** $\mathrm{p}<.01$ 
POST-HOC ANALYSES

\section{Total errors, Iong-term recall}

The total errors on the fifth trial were subjected to a $t$ test (Bruning \& Kintz, 1968, pp. 9-12). The difference between the two treatment groups was not significant at the .05 level $(\underline{t}=1.19$, $d f=22)$. The mean of the total errors for the crossed group was 2.83. The mean of the total errors for the nested group was 0.83 .

Structural errors, short-term recall

The errors made on the fifth trial were broken down into row, column, and within-column errors. These structural errors were then used to test the induction of structure hypothesis. These tests showed some significant long-term recall differences (see table VIII). Post-hoc analysis of the short-term recall structural errors was performed. Each of the row, column, and within-column errors of the first four trials were subjected to separate $2 \times 4$ (treatment mode $x$ trials) ANOVAs with repeated measures on the treatment mode variable using conservative degrees of freedom and post-hoc comparison on the trials factor (Winer, 1971, pp. 514-539).

There were no significant differences at the .05 level between the two treatment groups for any of the structural errors (see tables $I X, X$, and $X I)$. There were no significant differences at the .05 level for any of the structural error dependent variables across trials (see tables $I X, X$, and $X I$ ). There were no significant interactions at the .05 level between any of the structural error dependent variables and the trials factor (see tables $I X, X$, and XI). 
TABLE IX

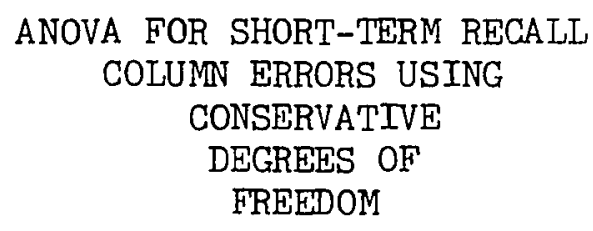

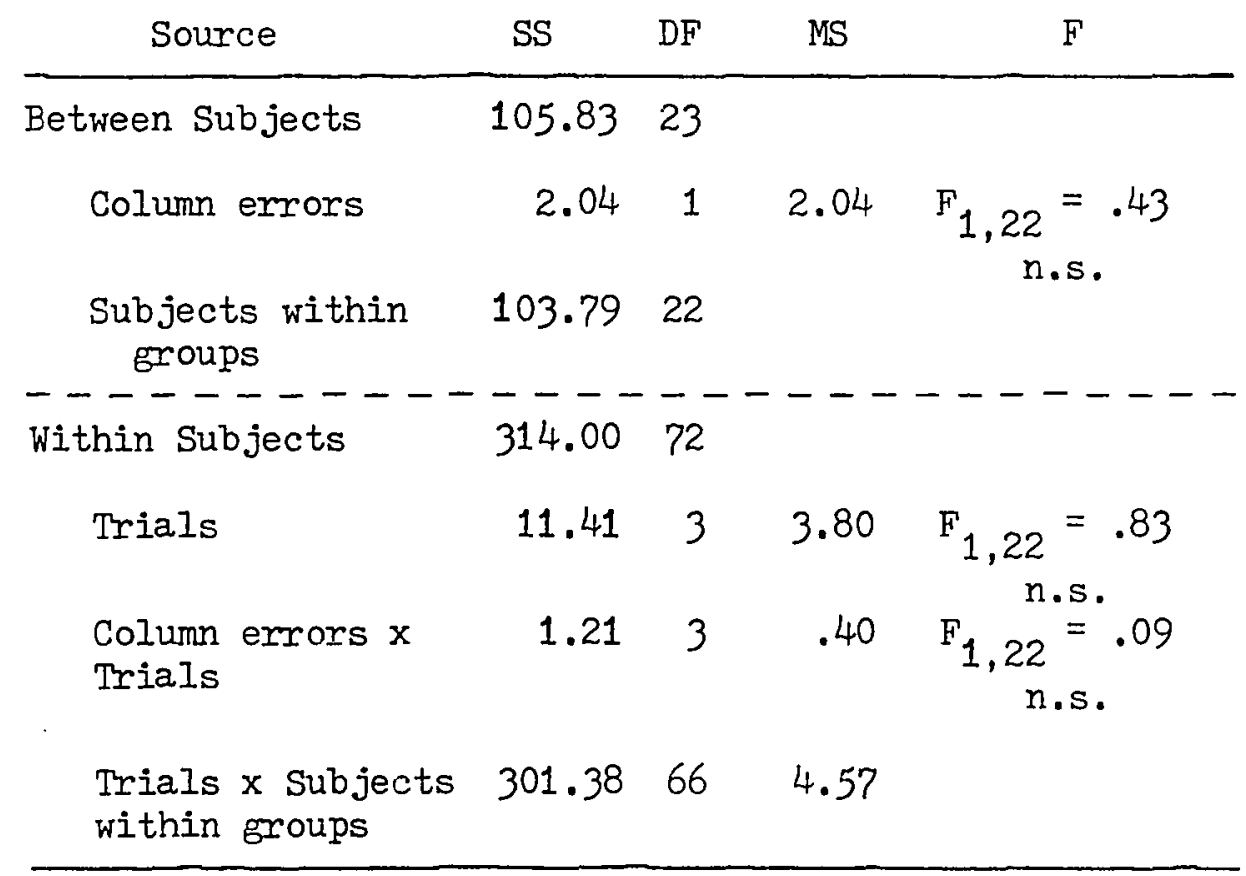


TABLE $X$

ANOVA FOR SHORT-TERM RECALL ROW

ERRORS USTNG CONSERVATIVE

DEGREES OF FREEDOM

\begin{tabular}{|c|c|c|c|c|c|}
\hline Source & SS & $\mathrm{DF}$ & MS & & $F$ \\
\hline Between Subjects & 246.96 & 23 & & & \\
\hline Row errors & 12.04 & 1 & 12.04 & $\mathrm{~F}_{1,22}$ & $\begin{array}{l}=1.13 \\
\text { n.s. }\end{array}$ \\
\hline $\begin{array}{l}\text { Subjects withir } \\
\text { groups }\end{array}$ & 234.92 & 22 & 10.68 & & \\
\hline $\begin{array}{l}------- \\
\text { Within Subjects }\end{array}$ & $-\frac{-}{213.00}$ & & -- & --- & --- \\
\hline Trials & 16.21 & 3 & 5.40 & $F_{1,22}$ & $\begin{array}{l}=1.91 \\
\text { n.s. }\end{array}$ \\
\hline $\begin{array}{l}\text { Row exrors } x \\
\text { Trials }\end{array}$ & 9.88 & 3 & 3.27 & $F_{1,22}$ & $\begin{array}{l}=1.16 \\
\text { n.s. }\end{array}$ \\
\hline $\begin{array}{l}\text { Trials x Sub- } \\
\text { jects within } \\
\text { groups }\end{array}$ & 186.91 & 66 & 2.83 & & \\
\hline
\end{tabular}


TABLE XI

$$
\begin{aligned}
& \text { ANOVA FOR SHORT-TERM RECALL } \\
& \text { WITHIN-COLUMN ERRORS } \\
& \text { USING CONSERVATIVE } \\
& \text { DEGREES OF } \\
& \text { FREEDOM }
\end{aligned}
$$

\begin{tabular}{|c|c|c|c|c|}
\hline Source & SS & $\mathrm{DF}$ & MS & $F$ \\
\hline Between Subjects & 12.49 & 23 & & \\
\hline $\begin{array}{l}\text { Within-column } \\
\text { errors }\end{array}$ & .84 & 1 & .84 & $F_{1,22}=1.58$ \\
\hline
\end{tabular}

\begin{tabular}{|c|c|c|c|c|}
\hline $\begin{array}{l}\text { Subjects within } \\
\text { groups } \\
\text { Within Subjects }\end{array}$ & $\begin{array}{l}11.65 \\
---75\end{array}$ & 72 & .53 & ----- \\
\hline Trials & 3.62 & 3 & 1.21 & $F_{1,22}=1.41$ \\
\hline $\begin{array}{l}\text { Within-column } \\
\text { errors x Trials }\end{array}$ & .36 & 3 & .12 & $\mathrm{~F}_{1,22}=.14$ \\
\hline $\begin{array}{l}\text { Trials x Subjects } \\
\text { within groups }\end{array}$ & 55.77 & 66 & .85 & \\
\hline
\end{tabular}

Absolute rearrangement

A second perspective on rearrangement can be taken. Rearrangement can be defined for a single dimension as the difference between memory input position and memory recall position. Call this "absolute rearrangement". As defined for the subject expectation effect, rearrangement variables measured the relative positions of pairs of dimensions in memory recall $\left(R_{1}=R-\right.$ Col). Input-recall differences are conceptually separate from dimensionaipair recall differences. Analysis of absolute rearrangement supplements the analysis of rearrangement. The correlation of absolute rearrangement with errors gives a second perspective on 
rearrangement.

Absolute rearrangement is defined as the difference between the first appearance of a dimension in the presentation sequence and the first appearance of that same dimension in the recall sequence. For example, the column dimension is always presented first to the subjects, so the presentation value is 1 . The absolute rearrangement measure for the column dimension is therefore 1 - Col. Let " $A_{i}^{n}$ " be defined as the $i^{\text {th }}$ measure of absolute rearrangement for the nested group. Let " $A_{i}^{c}$ " be defined as the $i^{\text {th }}$ measure of absolute rearrangement for the crossed group. Let "Col", "R", and "WC" be defined as they were for the rearrangement measures, i.e. as the first appearance of the respective dimensions. Let each $A_{i}$ be defined as they are in table XII. The error data and the absolute rearrangement data of the first four trials were subjected to Pearson product-moment correlations and significance tests (Bruning \& Kintz, 1968, pp. 152-155).

Absolute rearrangement errox correlations for short-term recall

The correlations between total errors and the absolute rearrangement measures are presented in table XII. The results show two correlations to be significant, one in each treament group. Both correlations involve the within-column dimension. For the crossed group the correlation between $A_{3}^{c}$ and crossed group total errors is significant at the .02 level $(z=2.42)$. For the nested group the correlation between $\mathrm{A}_{2}^{\mathrm{n}}$ and nested group total errors is significant at the .0001 level $(z=4.71)$. 


\section{TABLE XII}

PEARSON PRODUCT-MOMENT CORRELATIONS BETWEEN TOTAL ERRORS AND ABSOLUTE REARRANGEMENT FOR SHORT-TERM RECALL

\begin{tabular}{|c|c|c|c|c|}
\hline Group & $A_{i}$ & Definition & $r$ & Significance \\
\hline \multirow{3}{*}{ Crossed } & $\dot{A}_{1}^{C}$ & $1-\operatorname{Col}$ & -.26 & n.s. \\
\hline & $\mathrm{A}_{2}^{\mathrm{C}}$ & $10-R$ & -.07 & n.s. \\
\hline & $\mathrm{A}_{3}^{\mathrm{C}}$ & 10-WC & .35 & $\mathrm{p}<.02$ \\
\hline \multirow{3}{*}{ Nested } & $\mathrm{A}_{1}^{n}$ & $\begin{array}{c}----- \\
1-\mathrm{Col}\end{array}$ & $\begin{array}{l}--- \\
-.11\end{array}$ & $\begin{array}{c}------- \\
\text { n.s. }\end{array}$ \\
\hline & $A_{2}^{n}$ & 10-WC & .69 & $p<.0001$ \\
\hline & $\mathrm{A}_{3}^{\mathrm{n}}$ & $19-R$ & -.03 & n.s. \\
\hline
\end{tabular}

Rearrangement error correlations for long-term recall

The correlations between total errors and the two rearrangement measures were calculated for the fifth trial recall. These correlations were Pearson product-moment correlations (Bruning \& Kintz, 1968 , pp. 152-155). The results show no correlations significant at the .05 level between total errors and the rearrangement measures $\left(R_{i}\right)$ (see table XIII). There were also no correlations significant at the .05 level between total errors and the absolute rearrangement measures $\left(A_{i}\right)$ (see table XIV). 


\section{TABI, XIII}

PEARSON PRODUCT-MOMENT CORRELATIONS BETWEEN TOTAL ERRORS AND REARRANGEMENT MEASURES

FOR LONG-TERM RECALL

\begin{tabular}{cccc} 
Group & $R_{i}$ & $r$ & $\underline{t}^{\mathrm{a}}$ \\
\hline 1 & -.42 & -1.46 \\
Crossed & 2 & -.38 & -1.30 \\
---1 & 3 & -.25 & -.82 \\
Nested & 2 & -.31 & -1.03 \\
& 3 & .24 & .78 \\
\hline
\end{tabular}

a. $d f=10$, two-tailed test

\section{TABLE XIV}

PEARSON PRODUCT-MOMENT CORRELATIONS BETWEEN TO'IAL ERRORS AND ABSOLUTE

REARRANGEMENT FOR LONG-TERM RECAIL

\begin{tabular}{lccc} 
Group & $A_{i}$ & $r$ & $\underline{t}^{2}$ \\
\hline Crossed & 2 & .19 & .61 \\
----1 & 3 & .12 & .38 \\
Nested & 2 & .44 & 1.55 \\
& 3 & .13 & .41 \\
\hline
\end{tabular}

a. $d f=10$, two-tailed test 
SUBJECTS' SELF-REPORTS

of the 24 subjects, 22 gave self-reports. The experimenter neglected to ask two subjects for their self-reports. When asked if they used images, 19 indicated that they did, at least partially. Three subjects indicated they used some form of a word association system. All 22 subjects mentioned clustering the recall material to some extent. Some remarks along this line were as follows: "recall one of a group, key into rest", "used the order of presentation", and "in each column, after got first (dimension), easier to recall other two (dimensions)." 
CHAPTER IV

DISCUSSION

The following discussion is divided into four sections. The first section discusses the experimental hypotheses. The second section discusses the post-hoc analyses. The third section presents conclusions. The fourth section suggests refinements which might amplify, clarify, and extend the experimental findings.

\section{EXPERIMENTAL HYPOTHESES}

\section{(1) Error hypothesis}

Both treatment groups made the same number of errors on the first four trials (see table IV). It may be concluded that the experimental techniques do not produce a significant difference in the error rate for short-term recall of $3 \times 3$ crossed and 9 item nested classifications. The error equality could be due to a balance effect. The theoretically greater efficiency of the cross classification could be balanced by a greater tendency to use the nested classification. The tendency to use the nested could be die to either a natural tendency to use nested classifications, more practice with nested classifications, or both effects together. The error equality may also be due to a ceiling effect. The mean errcrs per trial was 2.2 with a standard deviation of 3.3 . 


\section{(2) Clustering hypothesis}

Both treatment groups clustered to the same extent on the first four trials (see table V). Clustering was, on the average, high for both groups, but varied widely. The mean clustering was .76 with a standard deviation of .26. The range of clustering was from 0 to 1 . The difference between the mean clustering and the presentation clustering value (of 1.00) was not significantly different at the .05 level using a $z$ test $(z=.92)$. Both groups did use a high degree of clustering, probably due to the large number of items (27) that were asked to be recalled. Any differences between the two treatment groups appears to have been wiped out by the large number of items.

\section{(3) Learning curve hypothesis}

For the error measure. neither group shored a learning curve (see table IV). This equality across trials could be due to a ceiling effect on the error rate or to the balance effect between higher crossed efficiency versus more nested practice.

For the clustering measure, neither group showed a different learning curve (see table V). Some one-trial learning did take place in the use of clustering, as shown by the post-hoc comparison of table VI. Overall, subjects quickly found their own optimal level of recall clustering.

(4) Induction of structure hypothesis

Some structure was induced in long-term memory. Both treatment groups on the fifth trial, as hypothesized, made the 
same number of errors when recalling the column dimension (see table VII). The nested group on the fifth trial, as hypothesized, made fewer errors than the crossed group when recalling the within-column dimensions. In fact, the nested group made no errors when recalling the column and within-column dimensions (see table VII). Contrary to hypothesis, on the fifth trial both groups recalled the row dimension equally poorly. It seems that the nested classification was induced in long-term memory by this procedure, but not the crossed classification.

Both treatment groups, on the trial after the intervening task, clustered their recall to the same extent. This is counter to the original hypothesis, but consistent with the subjects' performance on the first four trials.

\section{(5) Subject expectation effect}

It was hypothesized that a subject expectation effect would show up as correlations between errors and measures of rearrangement. There were indeed two significant correlations, both for the crossed group (see table VIII). Close examination of both of them showed that subjects' memory efficiency declined as their recall pattern departed further from the input pattern of the stimulus (see figures 17 and 18).

The value of $R_{2}^{c}$ in the presentation protocol is 18 . In figure 17 , as the value of $R_{2}^{c}$ declines, the within-column dimension is recalled closer and closer to the column dimension. Finally, when $R_{2}^{c}$ equals -1 , the within-column dimension is recalled before the 


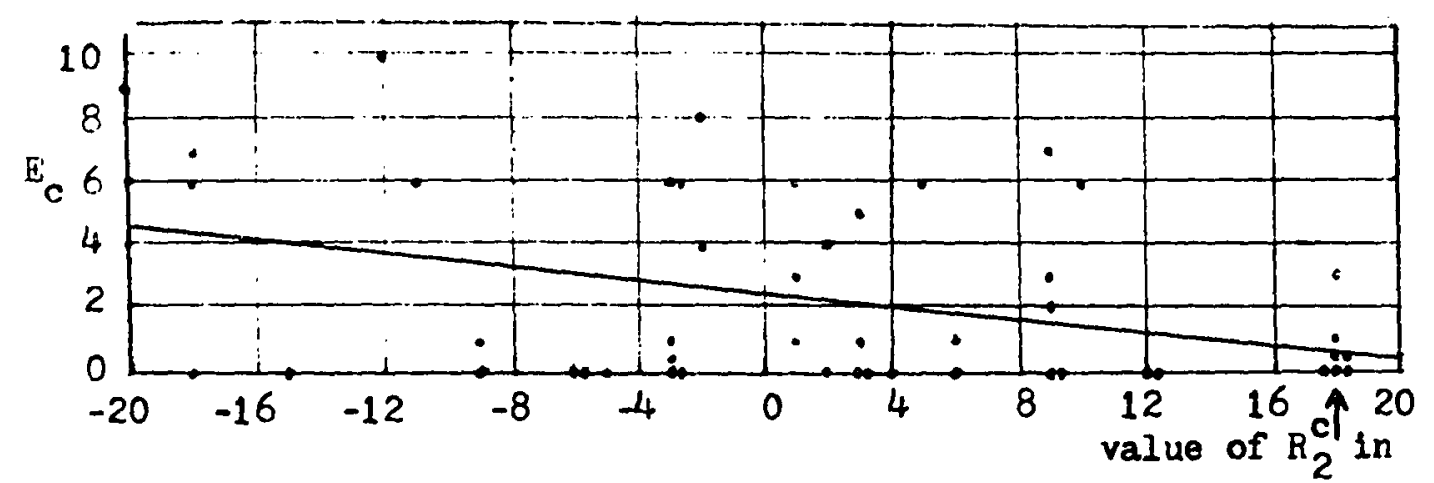

$$
R_{2}^{C}=W C-\text { Col } \quad \text { the presentation }
$$

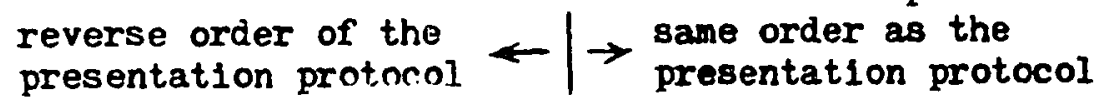

Figure 17. Scatter diagram and regression line for crossed group error data $\left(E_{c}\right)$ and the second rearrangement measure $\left(R_{2}^{c}\right)$ $\left(E_{c}=-.1 R_{2}^{c}+2.5\right)$.

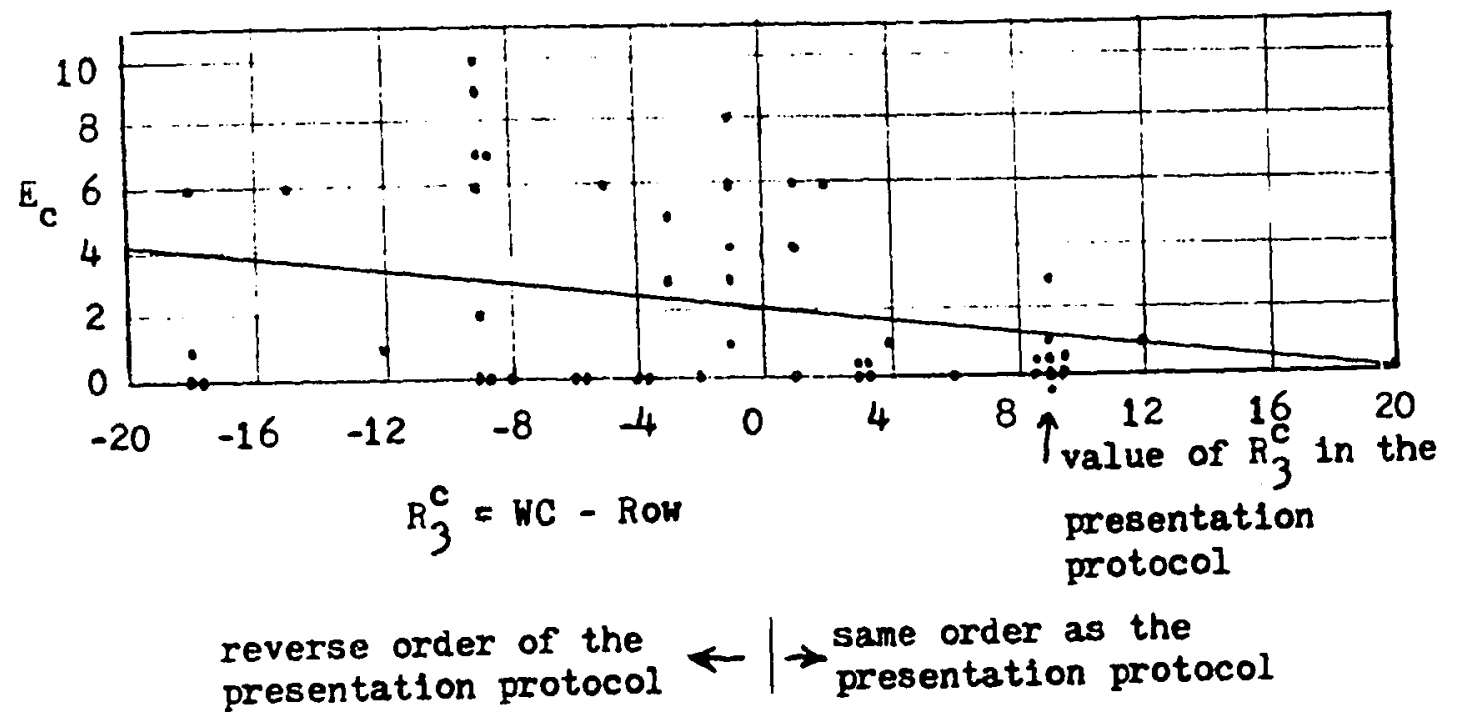

Figure 18. Scatter diagram and regression line for crossed group error data $\left(E_{c}\right)$ and the third rearrangement measure $\left(R_{3}^{c}\right)$ $\left(E_{c}=-.1 R_{3}^{c}+2.14\right)$.

column dimension in the recall sequence. As the value of $R_{2}^{c}$ becomes increasingly negative, the within-column dimension is recalled earlier in the recall sequence relative to the column dimension. 
As $R_{2}^{C}$ becomes negative, a reversal of the order of the presentation order occurs. As the subject departs from the order of presentation, memory efficiency declines. A similar line of reasoning holds for the correlation between $\mathrm{R}_{3}^{\mathrm{C}}$ and $\mathrm{E}_{\mathrm{C}}$.

It appears that the crossed group rearranged material in their memories in a destructive manner whereas the nested group did not. Moreover, since the within-column dimension appears in both $\mathrm{R}_{2}^{\mathrm{C}}$ and $R_{3}^{c}$, this dimension appears to be the dismuptive element. Thus a bias against the presentation order either did not help or else actively hindered recall. It appears that a subject expectation effect might be present for some subjects. These conclusions are somewhat speculative, being based only on the correlations.

\section{POST-HOC ANALYSES}

Absolute rearrangement error correlations for short-term recall

Two absolute rearrangement measures, one for each treatment group, correlated significantly with errors (see table XII). Closer examination of these two significant correlations (see figures 19 and 20) showed the same pattern for both groups. As the within-column dimension appeared earlier and earlier in the recall sequence, errors increased.

The horizontal axes of figures 19 and 20 are expressed in terms of both the absolute rearrangement measures and the position of the within-column dimension in the recall sequence. The regression equations are similarly calculated using both of these 
variables. The correlations of the group errors with the positions have the same absolute values as, but opposite signs from, the respective correlations with the absolute rearrangement. This is due to the definition of absolute rearrangement, which consists of a constant minus a position value of a single dimension (see table XII). The correlations between errors and absolute rearrangement are the correlations between errors and position with a sign change.

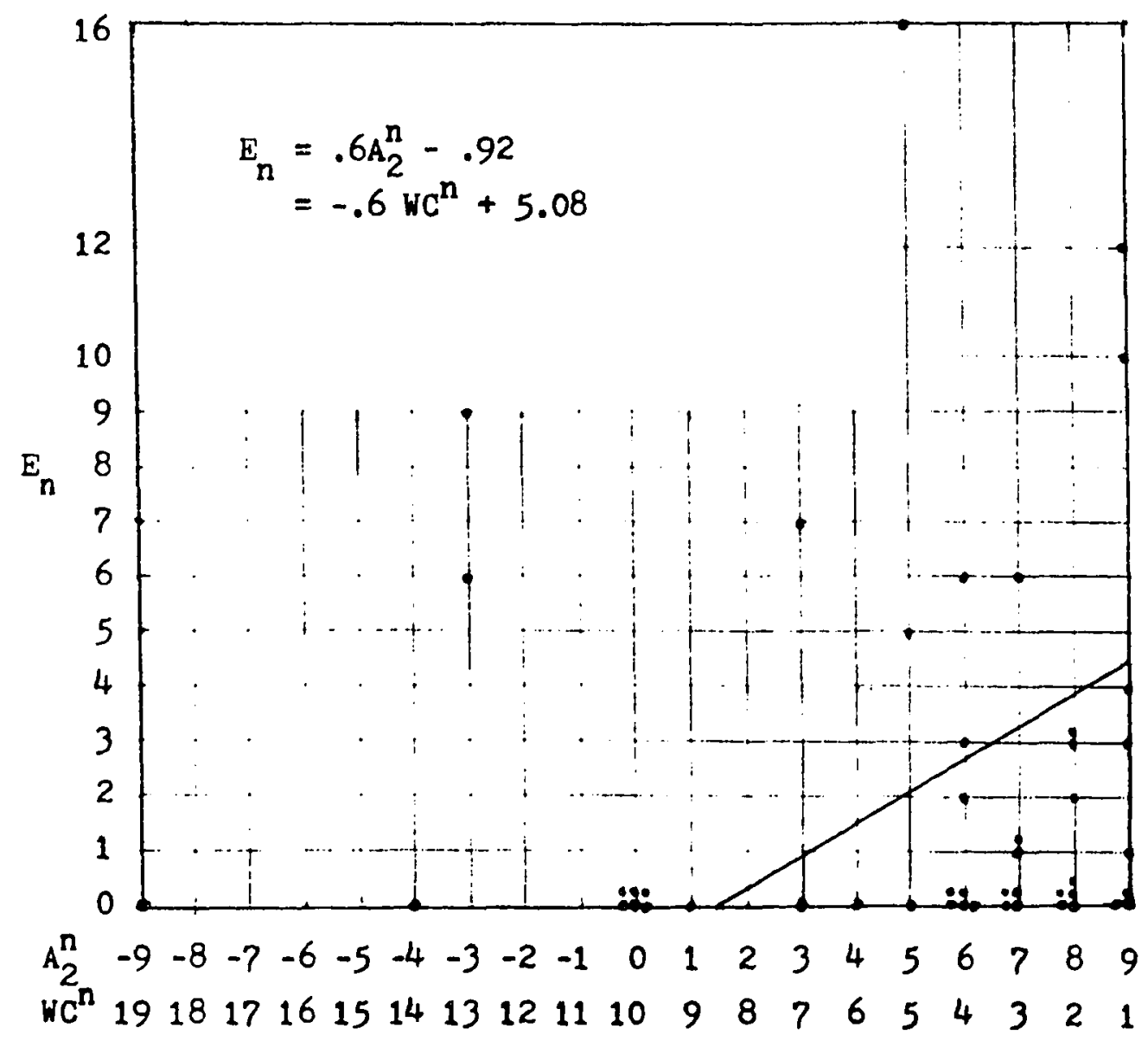

Figure 19. Scatter diagram and regression line for nested group error data $\left(E_{n}\right)$ with the second absolute rearrangement measure $\left(A_{2}^{n}\right)$ and with the within-column dimension first appearance $\left(W C^{n}\right)$. 


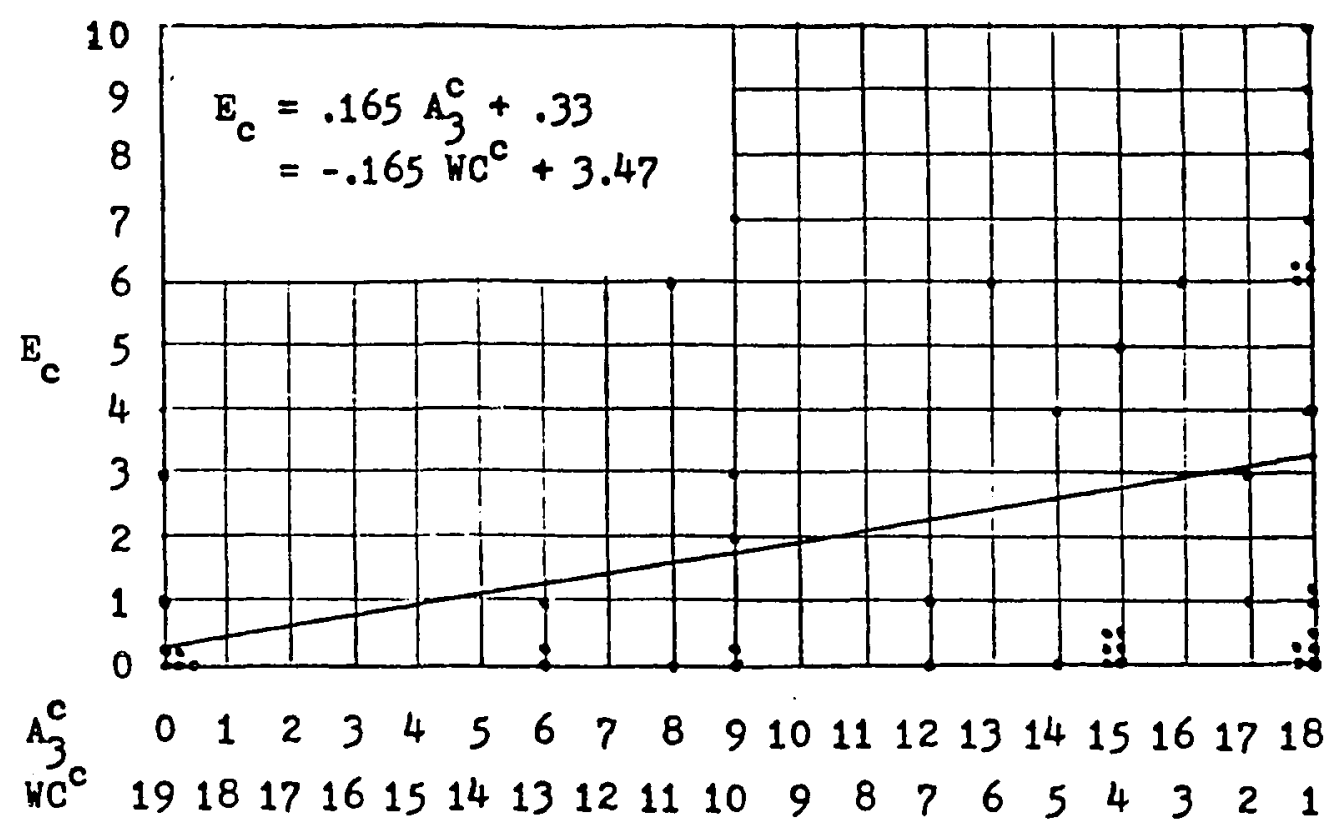

Figure 20. Scatter diagram and regression line for crossed Eroup error data $\left(E_{c}\right)$ with the third absolute rearrangement measure $\left(A_{3}^{C}\right)$ and with the within-column dimension first appearance $\left(W C^{C}\right)$.

The short-term recall total errors of both groups appear to be a function of the recall position of the within-column dimension. The absolute rearrangement correlations are consistent with this hypothesis. The $R_{i}$ s are significantly correlated with the total errors only for the crossed group. The correlations provide some support for the hypothesis that the total errors in short-term recall are caused by the position of the within-column dimension in the recall sequence.

\section{Rearrangement error correlations for long-term recall}

Neither the absolute rearrangement variables $\left(A_{1}\right)$ nor the rearrangement measures $\left(R_{1}\right)$ correlated significantly with total errors for long-term recall (see tables XIII and XIV). Short-term 
total errors did correlate significantly with both types of rearrangement measures. This short-term long-term correlation difference could be due to a difference between the stability of short-term and Iong-term memory. Once the stimulus attributes had been rearranged while passing through short-term memory, they became fixed in long-term memory. The correlations of rearrangement with errors would then drop out in long-term recall, as subjects would have access to the attributes in a stable listing.

Total errors, long-term recall

Both treatment groups made the same number of total errors on the fifth trial. Whatever factors were responsible for the equality of errors between the two groups in short-term recall, appears also to have been operative in long-term recall.

\section{Stmuctural errors, short-term recall}

Both treatment groups made the same number of structural errors on the first four trials (see tables IX, X, and XI). This error equality may be due to a ceiling effect which masks the kind of structural error differences that were revealed for long-term recall.

\section{CONCLUSIONS}

The experimental results and the post-hoc analyses give support to the hypothesis that the nested classification tends to override the crossed classification when the two are in direct competition to organize material for recall. Three findings support 
this hypothesis. The nested structure could be induced in lonc-term memory, but the crassed structure tended not to be induced. This was shown by perfect recall of the nested structures by the nested group and the poor recall of the crossed stmuctures by the crossed group in long-term recall (see table VII). Nested structures tend to disrupt recall in short-term memory. The within-column dimensions are structures of the nested classification. When the within-column dimensions were recalled first, more recall errors were made, especially for the crossed group. This finding was based on the correlations between total errors and the absolute rearrangement measures (see table XII and figures 19 and 20) and by the correlations between total errors and the rearrangement measures (see table VIII and ficures 17 and 18). The results are consistent with the hypothesis that the nested classification tends to override the cross classification. Overall, the experimental results provide evidence for the hypothesis that the experimental procedure can induce the memory for nested classifications but not the memory for cross classifications.

\section{IMPLICATIONS FOR FURTHER RESEARCH}

\section{Amplification}

Several changes in the experimental procedure might amplify the differences between crossed and nested treatments enough to make those differences statistically significant.

1. The sample size can be increased. This would have two consequences. Any statistical analyses would be more sensitive, 
hence increasine, the chances of pickine up an effect. AlI of the stimulus dimensions could be included, eliminating them as a possible source of experimental variation.

2. One subject remarked that he could easily recall the stimulus set after the intervening task because he had already written it down once. In the revised experiments, the subjects should have to perform the intervening task before recall of the last stimulus set. This should amplify any memory loss.

3. Another way of amplifying memory loss would be to have subjects recall the first stimulus set after they had worked with several subsequent sets.

4. The clustering data indicate substantial one-trial learning, which may be two-trial learning if the practice set is included. A refined experiment could thereby use fewer trials and more subjects in the same amount of time as this thesis study needed. Hopefully, these four changes would bring forth differences between treatments at a statistically significant level.

\section{Clarification}

Several changes in the experimental procedure would clear up procedural difficulties.

1. The physical format (defined on p. 33) of the stimulus sets and recall sheets could be manipulated as a third factor in addition to the trials and treatment factor. This manipulation would unconfound the physical format from the order of presentation. To do so in this thesis study would have required a subject population 
four times the size that was used. Perhaps a later study might simply drop the physical format variable if it proves too costly. 2. The stimulus sets could be improved so that their dimensions were more distinguishable. Some subjects remarked that they confused the bottle heights and the bottle neck lengths because both modes referred to length. However, other subjects remarked that recall of one of these dimensions facilitated recall of the other. Still others remarked that the width dimension was especially difficult to recall. If other, more distinct, dimensions could be found, the effects noted by these subjects could be reduced.

3. The stimulus sets could be improved so that redundancy between sets would be diminished. Some subjects remarked that they felt their memory task was made easier because several sets used the same dimensions. These were mostly height, width, and number. In particular, subjects mentioned this when these variables appeared in successive sets as row or column dimensions. An alternative procedure would be to match the sets carefully so that these redundant stimulus dimensions do not appear in row and column positions more than once for any subject.

4. The stimulus sets could be improved so that the orderliness of some of the stimulus dimensions was increased. Some subjects noted that some of the stimulus dimensions were not as well ordered as others, for example, roof shape or door shape. This differential orderliness made some subjects feel these dimensions were more difficult to recall. Equalizing the orderliness of the dimensions seems desirable also from the theoretical standpoint. 
5. The possibility exists that the nested group may be getting more practice on the row attributes than the crossed group. The nested group is asked to repeat the row attributes three times apiece. The crossed group is asked to repeat the row attributes only once. While no differences showed up in row errors between the two groups, this procedural difference should be corrected by having the crossed group repeat the row attributes three times. These changes would clarify and clean up some procedural difficulties in the experiment.

Extension

1. The experimental procedure could be extended to include more dimensions so that the crossed structure comes to have a greater advantage over the nested. For example, a $3 \times 3 \times 3$ matrix requires 3 dimensions as a crossed structure but 27 dimensions as a nested structure.

2. Different orders of presentation could be tried. There are six possible orders of presentation among the column, row, and the within-column dimensions (considering the within-column dimensions as one group).

3. The within-column dimensions could be tried in the row positions to see if any effects attributable to them might be due to a set to orient recall toward vertical orderings.

4. The row attributes could be presented to the nested group in the same way as to the crossed group, i.e. as rows, not as within-column differences. This procedural change would make the 
experiment more one of competition between the two classifications rather than an experiment which tries to induce structure. All these suggestions open up new aspects of the problem of memory for crossed and nested classifications, a problem which this thesis has begun to probe. 


\section{REFERENCES}

Anderson, Barry F. The Psychology Experiment. Brooks/Cole Publishing Co., a Division of Wadsworth Phblishing $\mathrm{Cn} ., \mathrm{Ca} .$, 1971.

Bousfield, W.A., Cohen, B.H., \& Whitmarsh, G.A. Associative clustering in the recall of words of different taxonomic frequencies of occurrence. Psychological Reports, 1958, 4. 39-44.

Bruning, James, \& Kintz, B.L. Computational Handbook of Statistics. Scott, Foresman and Co., Glenview, Illinois, 1968.

Cohen, B.H., Sakoda, J.M., \& Bousfield, W.A. The statistical analysis of the incidence of clustering in the recall of randomly arranged associates. Technical Report No. 10. ONR Contract Nonr-631(000), University of Connecticut, 1954.

Collins, A.M., \& Quillian, M.R. Retrieval time from semantic memory. Journal of Verbal Learning and Verbal Behavior, 1969, 8, 240-247.

Diogenes. The April Game. Playboy Press, Chicago, Illinois, 1973.

Frender, Robert, \& Doubilet, Peter. More on measures of category clustering in free recall-although probably not the last word. Psychological Bulletin, 1974, 81(1), 64-66.

Hays, William L. Statistics for the Social Sciences. Holt, Rinehart and Winston, N.Y., N.Y., 1973.

Klatzky, Roberta L. Human Memory. W.H. Freeman and Co., San Francisco, Ca., 1975.

Levy, Charles K. Elements of Biology. Appleton-Century-Crofts, Educational Division, Meredith Co., N.Y., N.Y., 1973.

Rosenthal, Robert. Experimenter Effects in Behavioral Research. Appleton-Century-Crofts, Educational Division, Meredith Co., N.Y., N.Y., 1966.

Winer, Ben J. Statistical Principles in Experimental Design, 2nd ed. McGraw-Hill, N.Y., N.Y., 1971. 


\section{APPENDIX A}

EXPERIMENTAI INSTRUCTIONS

\section{Instructions}

This is a memory experiment. You will be asked to memorize and recall five sets of pictures. You can practice on the first set. Your memory will them be exercised on the other four sets. You will also be asked to recall the last set twice.

I have summarized below the procedure you will go through with each set of pictures. The procedure is divided into two parts, practice and recall.

Practice

1. I will show you the pictures.

2. I will ask you to describe the different parts of the pictures until the complete set is described.

3. I will ask you to describe the pictures a second time.

Recall

1. I will remove the pictures and give you a blank form.

2. You will write down your descriptions of the pictures on the blank form in the appropriate places. As you write down your words, I will be marking beside them the order in which you write them down. 
This is the experimental procedure. If you have no questions, we will begin with the practice picture set. 
APPENDIX B

PRESENTATION ORDERS

\section{Crossed protocol}

1. The (content) in the first column all have the same (column dimension). What will you call this (column dimension)?

2. The (content) in the second column all have the same (column dimension). What will you call this (column dimension)?

3. The (content) in the third column all have the same (column dimension). What will you call this (column dimension)?

4. The (content) in the first row all have the same (row dimension). What will you call this (row dimension)?

5. The (content) in the second row all have the same (row dimension). What will you call this (row dimension)?

6. The (content) in the third row all have the same (row dimension). What will you call this (row dimension)?

7. The (content) in the first column all have different (first within-column dimension). What will you call these (first withincolumn dimension)?

8. The (content) in the second column all have different (second within-column dimension). What will you call these (second within-column dimension)?

9. The (content) in the third column all have different (third within-column dimension). What will you call these (third within-column dimension)?

10. The (content) in the first column are all ...?

11. The (content) in the second column are all ...?

12. The (content) in the third column are all . . ?

13. The (content) in the first row are all .. . ?

14. The (content) in the second row are all...?

15. The (content) in the third row are all ...?

16. The (content) in the first column have different (first withincolumn dimension). They are .. . ? 
17. The (content) in the second column have different (second withincolumn dimension). They are...?

18. The (content) in the third column have different (third withincolumn dimension). They are...?

\section{Nested protocol}

1. The (content) in the first column all have the same (column dimension). What will you call this (column dimension)?

2. The (content) in the second column all have the same (column dimension). What will you call this (column dimension)?

3. The (content) in the third column all have the same (column dimension). What will you call this (column dimension)?

4. The (content) in the first column all have different (first within-column dimension). What will you call these (first within-column dimension)?

5. The (content) in the second column all have different (second within-column dimension). What will you call these (second within-column dimension)?

6. The (content) in the third column all have different (third within-column dimensisn). What will you call these (third withincolumn dimension)?

7. The (content) in the first column all have different (row dimension). What will you call these (row dimension)?

8. The (content) in the second column all have different (row dimension). What will you call these (row dimension)?

9. The (content) in the third column all have different (row dimension). What will you call these (row dimension)?

10. The (content) in the first column are all ...?

11. The (content) in the second column are all ...?

12. The (content) in the third column are all . . . ?

13. The (content) in the first column have different (first withincolumn dimension). They are... ?

14. The (content) in the second column have different (second withincolumn dimension). They are... ? 
15. The (content) in the third column have different (third withincolumn dimension). They are...?

16. The (content) in the first column have different (row dimension). They are...?

17. The (content) in the second column have different (row dimension). They are...?

18. The (content) in the third column have different (row dimension). They are...? 


\section{APPENDIX C}

RECALI SHEETS

Nested recall sheets (reduced)

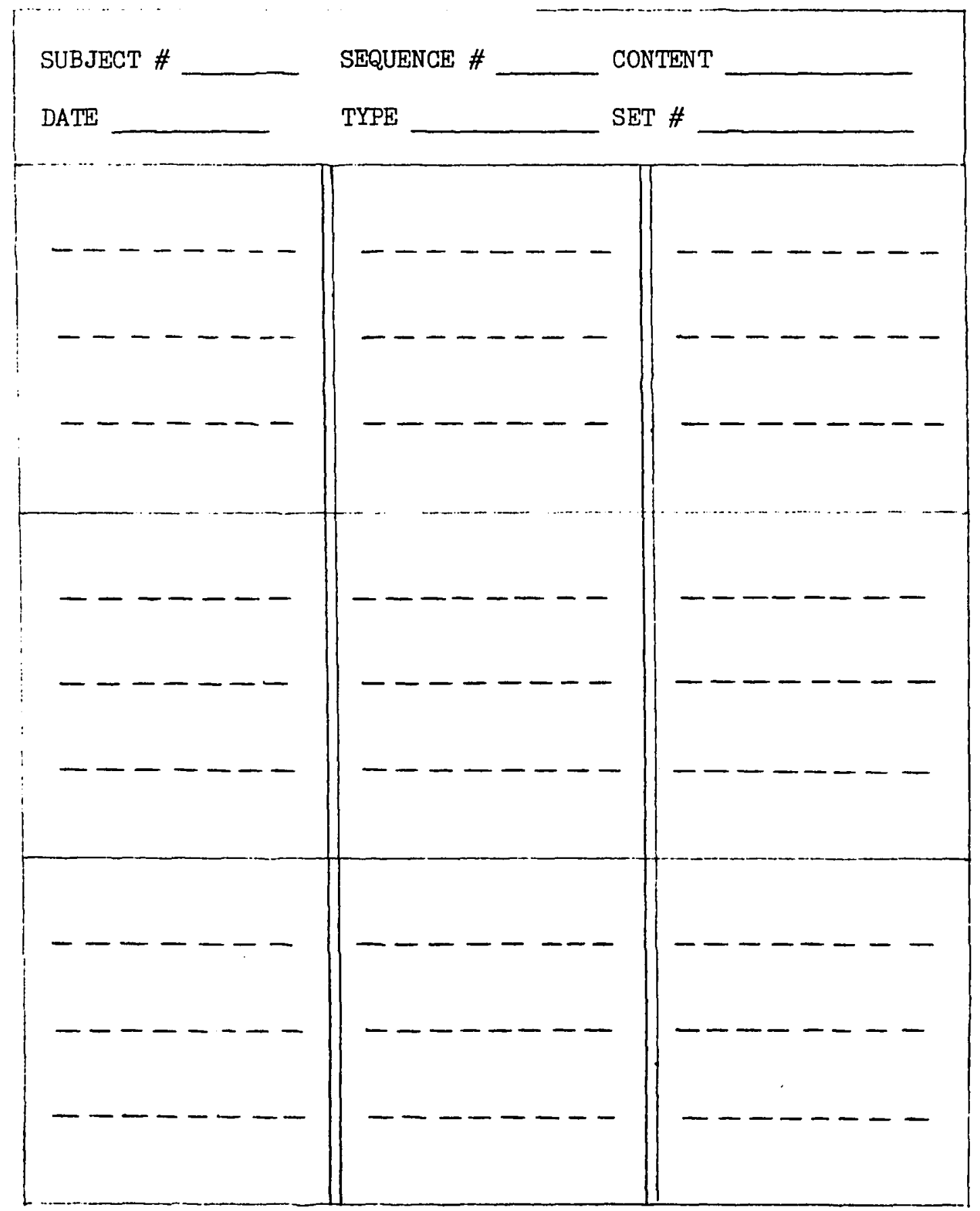


Crossed recall sheet (reduced)

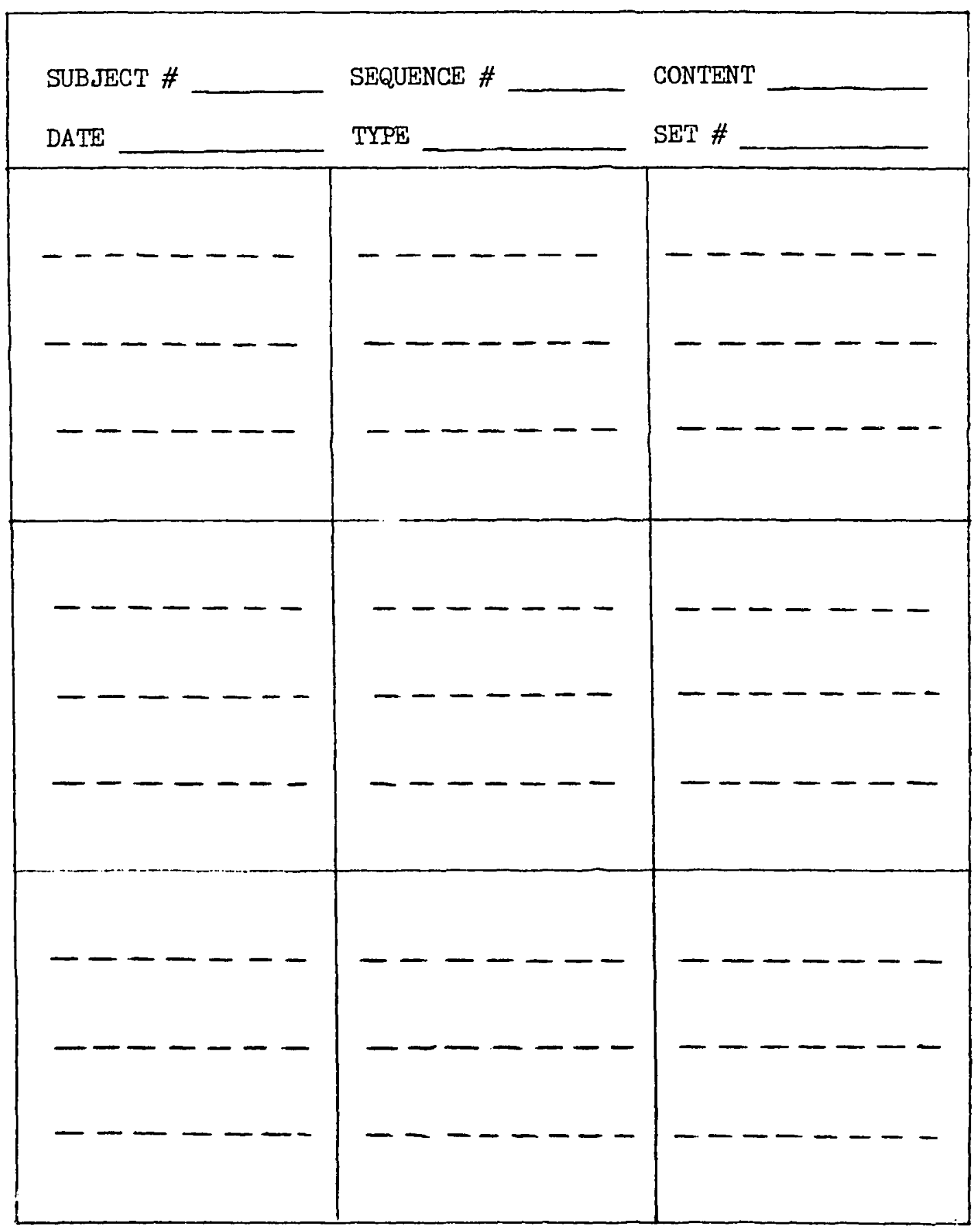


APPENDIX D

PHYSICAL FORMATS OF THE STIMULUS SETS 
Crossed format (reduced)

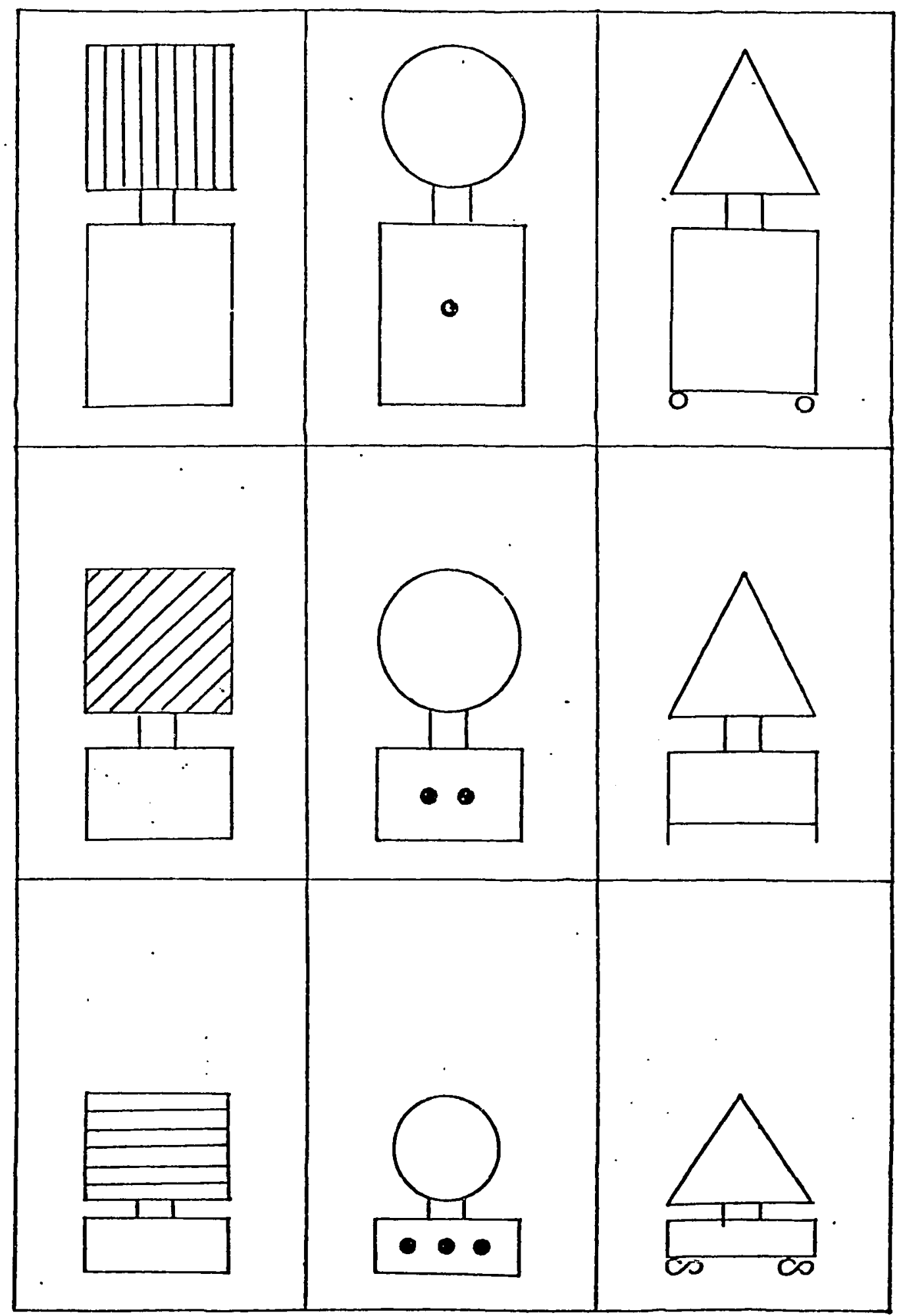


Nested format (reduced).

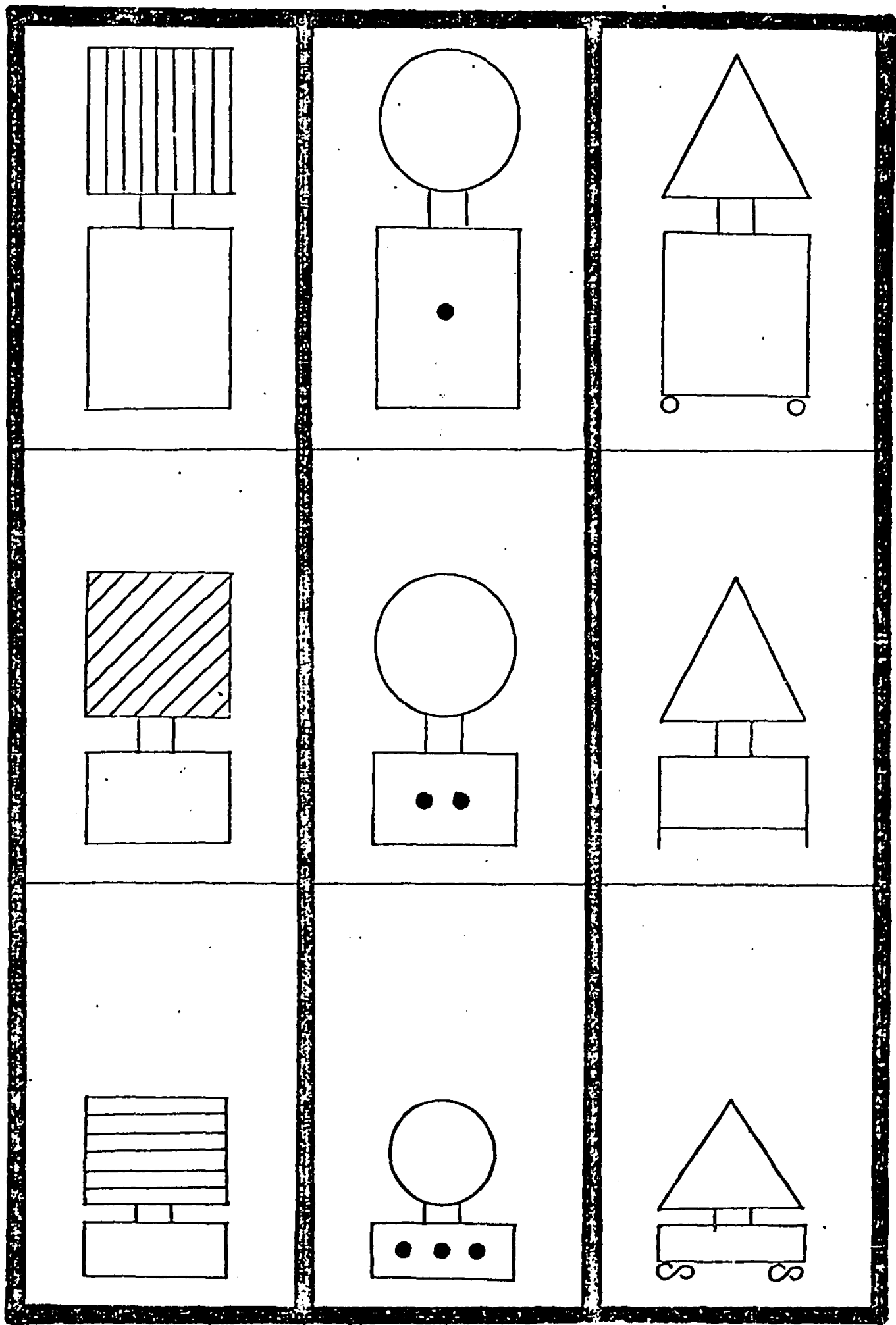




\begin{abstract}
APPENDIX E
PRACTICE STIMULI CONTENT MODES, LABELS, CONTENT MODE ROTATIONS AND

STIMULUS SETS
\end{abstract}

TABI.E XY

PRACTICE STIMLI CONTENT MODES AND IABELS

\begin{tabular}{l|c}
\hline Content Mode & Label \\
\hline Lamp height & $\mathrm{H}$ \\
\hline Shade shape & $\mathrm{S}$ \\
\hline Shade striping & $\mathrm{St}$ \\
\hline Number of dots & $\mathrm{D}$ \\
\hline Foot type & $\mathrm{F}$ \\
\hline
\end{tabular}

TABLE XVI

PRACTICE STIMULI CONTENT MODE ROTATIONS

\begin{tabular}{|c|c|c|c|c|c|c|}
\hline \multirow{2}{*}{\multicolumn{2}{|c|}{ Dimension }} & \multicolumn{5}{|c|}{ Stimulus set number } \\
\hline & & 1 & 2 & 3 & 4 & 5 \\
\hline \multicolumn{2}{|l|}{ Row } & $\mathrm{H}$ & $F$ & $\mathrm{D}$ & st & $S$ \\
\hline \multicolumn{2}{|l|}{ Column } & $S$ & $\mathrm{H}$ & $F$ & $\mathrm{D}$ & St \\
\hline \multirow{3}{*}{$\begin{array}{l}\text { Within- } \\
\text { Column }\end{array}$} & Ist & St & $\mathrm{S}$ & $\mathrm{H}$ & $F$ & D \\
\hline & 2nd & $D$ & st & $S$ & $\mathrm{H}$ & $F$ \\
\hline & $3 r d$ & $\mathrm{~F}$ & $D$ & St & $S$ & $\mathrm{H}$ \\
\hline
\end{tabular}


Practice stimulus sets (reduced)






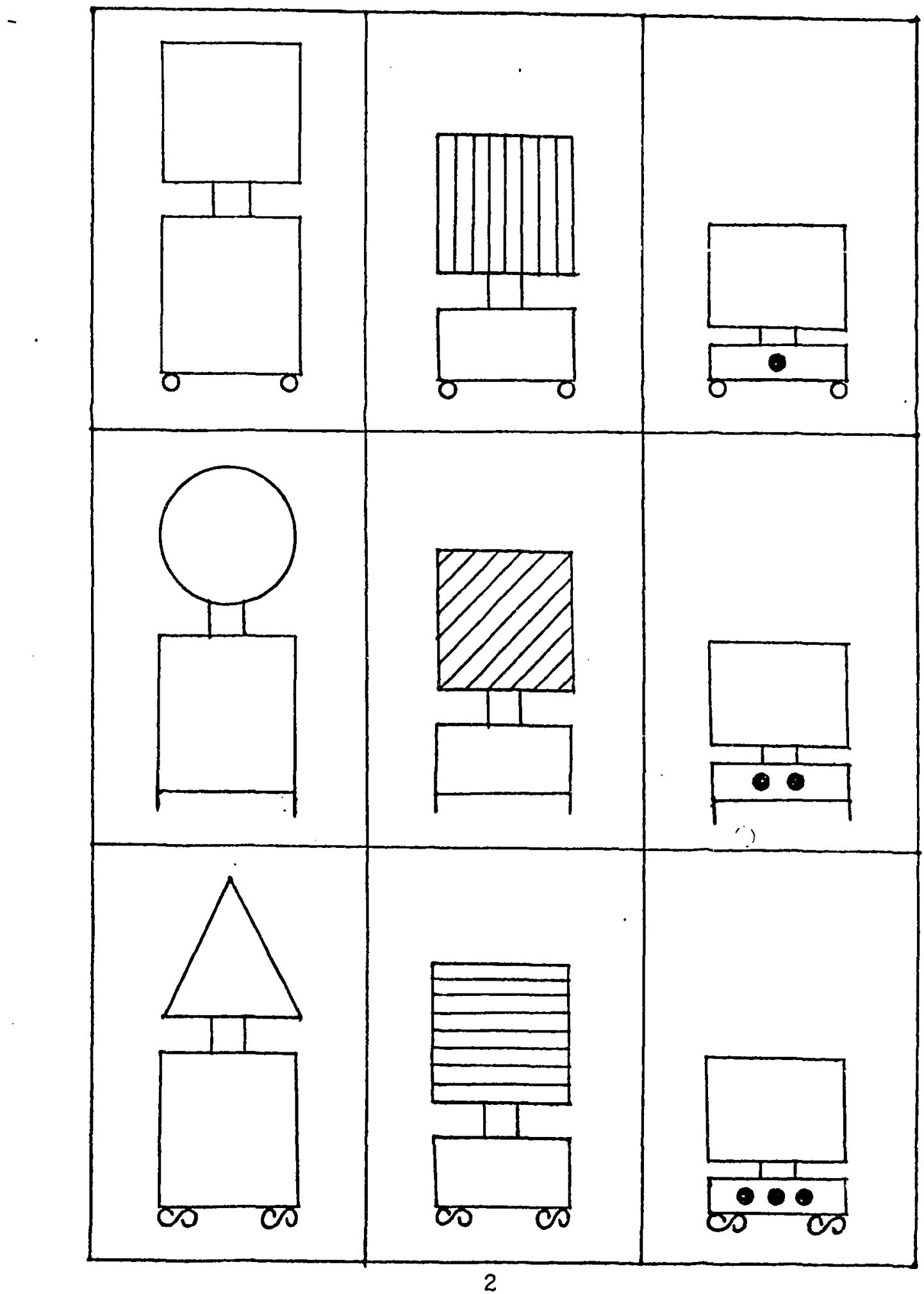




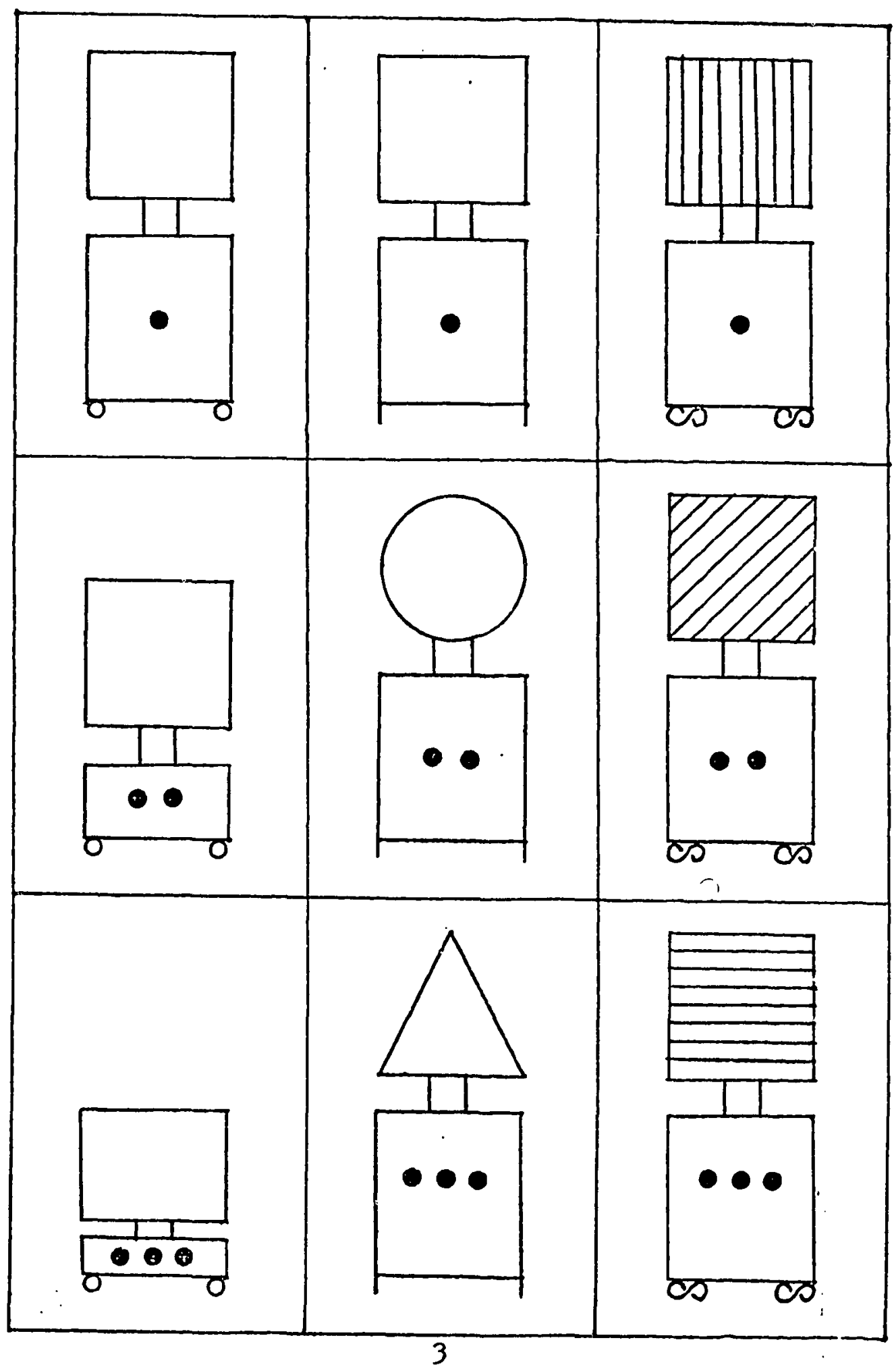




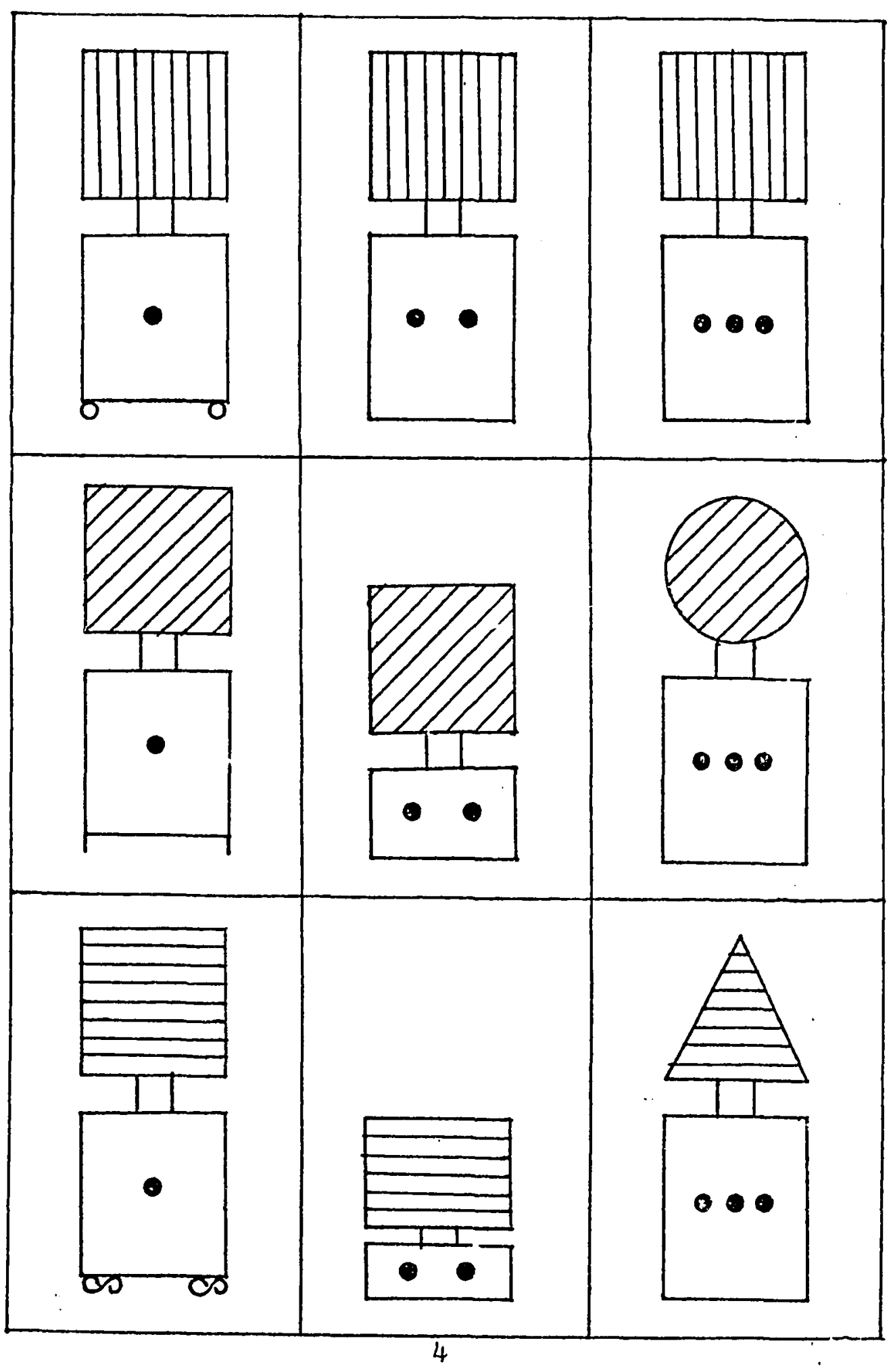




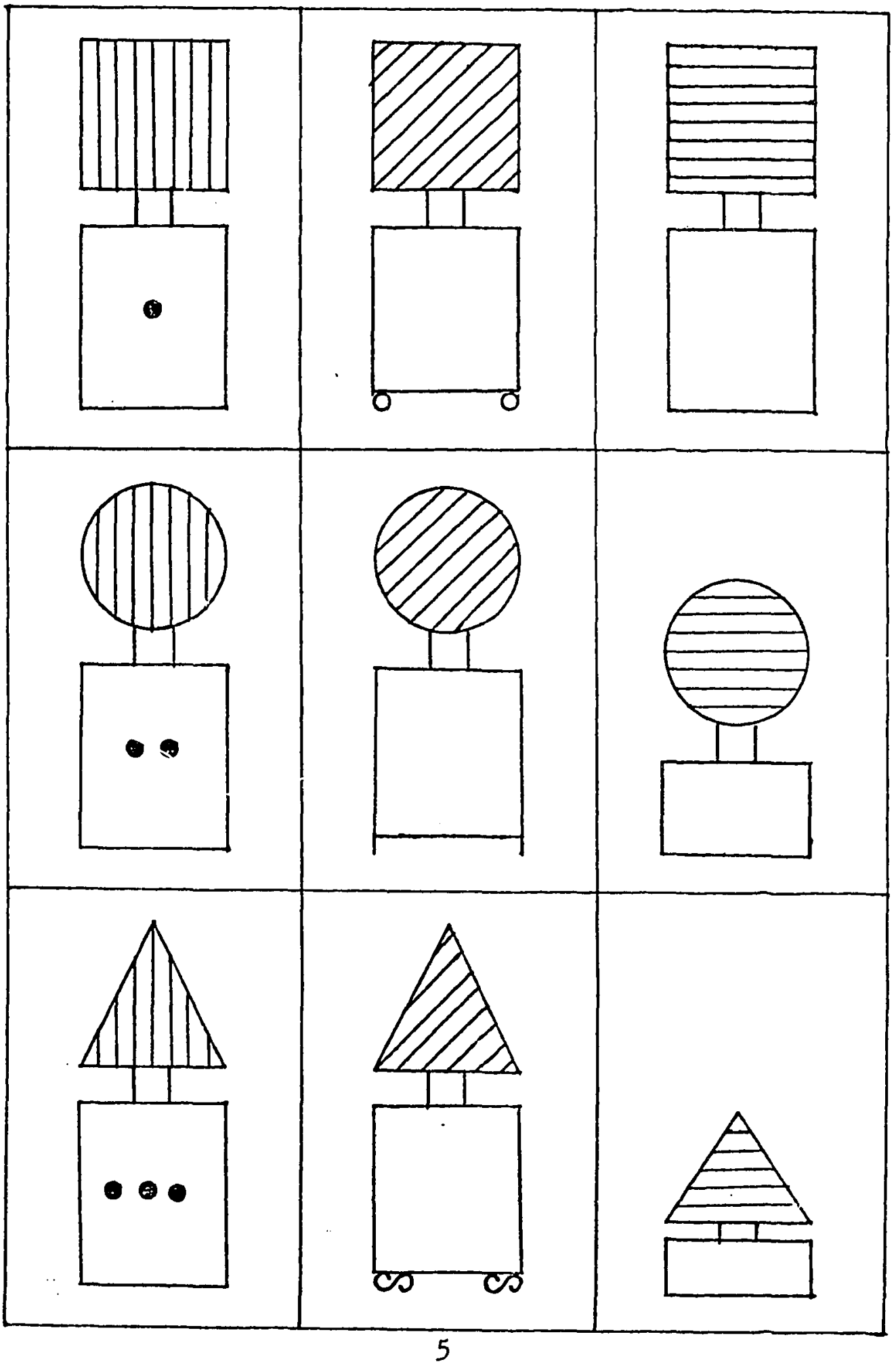




\section{APPENDIX F}

STIMULUS CONTENTS, CONTENT MODES, LABELS, ROTATIONS, AND STIMULUS SETS 
TABLE XVII

STIMULUS CONTENT MODES, LABELS, ROTATIONS

Mountain Stimulus set

\begin{tabular}{|c|c|c|c|c|c|c|c|c|}
\hline \multirow[t]{2}{*}{ Content mode } & \multirow[t]{2}{*}{ Label } & \multirow{2}{*}{\multicolumn{2}{|c|}{ Dimension }} & \multicolumn{5}{|c|}{ Stimulus set number } \\
\hline & & & & 1 & 2 & 3 & 4 & 5 \\
\hline Width & W & \multicolumn{2}{|l|}{ Row } & W & $\mathbf{S}$ & C & $\mathrm{H}$ & $\mathrm{P}$ \\
\hline Peak number & $P$ & \multicolumn{2}{|l|}{ Column } & $\mathrm{P}$ & $W$ & $\mathbf{S}$ & C & $\mathrm{H}$ \\
\hline Height & $\mathrm{H}$ & \multirow{3}{*}{$\begin{array}{l}\text { Within } \\
\text { column }\end{array}$} & 1st & $\mathrm{H}$ & $P$ & $W$ & $s$ & C \\
\hline Cloud number & C & & 2nd & $\mathrm{C}$ & $\mathrm{H}$ & $\mathbf{P}$ & $W$ & S \\
\hline Snow amount & $S$ & & $3 x d$ & $\mathrm{~S}$ & $\mathrm{C}$ & $\mathrm{H}$ & $\mathrm{P}$ & $W$ \\
\hline
\end{tabular}

Letter A Stimulus set

\begin{tabular}{|c|c|c|c|c|c|c|c|c|}
\hline Iine thickless & $\mathbf{T}$ & Row & & $\mathrm{T}$ & $W$ & $\mathrm{H}$ & $F$ & $\mathrm{P}$ \\
\hline Peak shape & $\mathbf{P}$ & Column & & $\mathbf{P}$ & $\mathrm{T}$ & $W$ & $\mathrm{H}$ & F \\
\hline Foot shape & $\mathbf{F}$ & \multirow{3}{*}{$\begin{array}{l}\text { Within } \\
\text { Column }\end{array}$} & $1 \mathrm{st}$ & $F$ & $\mathbf{P}$ & $\mathrm{T}$ & W & $\mathrm{H}$ \\
\hline Height & $\mathrm{H}$ & & 2nd & $\mathrm{H}$ & $\mathrm{F}$ & $\mathbf{P}$ & $\mathrm{T}$ & W \\
\hline Letter width & $W$ & & $3 r d$ & $W$ & $\mathrm{H}$ & $\mathrm{F}$ & $\mathbf{P}$ & $T$ \\
\hline
\end{tabular}

Bottle Stimulus Set

\begin{tabular}{|c|c|c|c|c|c|c|c|c|}
\hline Neck length & $\mathrm{N}$ & Row & & $\mathrm{N}$ & $W$ & L & $F$ & $\mathrm{H}$ \\
\hline Height & $\mathrm{H}$ & Column & & $\mathrm{H}$ & $\mathrm{N}$ & W & $\mathrm{L}$ & $F$ \\
\hline Fluid level & $F$ & \multirow{3}{*}{$\begin{array}{l}\text { Within } \\
\text { Column }\end{array}$} & $1 \mathrm{st}$ & $F$ & $\mathrm{H}$ & $\mathrm{N}$ & $W$ & $\mathrm{~L}$ \\
\hline Label & I & & 2nd & L & $F$ & $\mathrm{H}$ & $\mathbf{N}$ & $W$ \\
\hline Width & $W$ & & $3 r d$ & W & $I$ & $F$ & $\mathrm{H}$ & $\mathrm{N}$ \\
\hline
\end{tabular}


House Stimulus Set

\begin{tabular}{|c|c|c|c|c|c|c|c|c|}
\hline \multirow{3}{*}{$\begin{array}{l}\text { Content mode } \\
\text { Height } \\
\end{array}$} & \multirow{3}{*}{$\begin{array}{c}\text { Label } \\
\mathrm{H} \\
\end{array}$} & \multirow{2}{*}{\multicolumn{2}{|c|}{ Dimension }} & \multicolumn{5}{|c|}{ Stimulus set number } \\
\hline & & & & 1 & 2 & 3 & 4 & 5 \\
\hline & & \multicolumn{2}{|l|}{ Row } & $\mathrm{H}$ & $\mathrm{D}$ & $W$ & Wn & $\mathrm{R}$ \\
\hline Roof shape & $\mathrm{R}$ & \multicolumn{2}{|l|}{ Column } & $\mathrm{R}$ & $\mathrm{H}$ & $\mathrm{D}$ & W & Wn \\
\hline Windows & Wn & \multirow{3}{*}{$\begin{array}{l}\text { Within } \\
\text { Column }\end{array}$} & 1st & Wn & $\mathrm{R}$ & $\mathrm{H}$ & D & $W$ \\
\hline Width & W & & 2nd & $W$ & Wn & $R$ & $\mathrm{H}$ & $\mathrm{D}$ \\
\hline Doors & $\mathrm{D}$ & & $3 r d$ & $\mathrm{D}$ & $W$ & Wn & $\mathrm{R}$ & $\mathrm{H}$ \\
\hline
\end{tabular}


Stimulus sets (reduced)

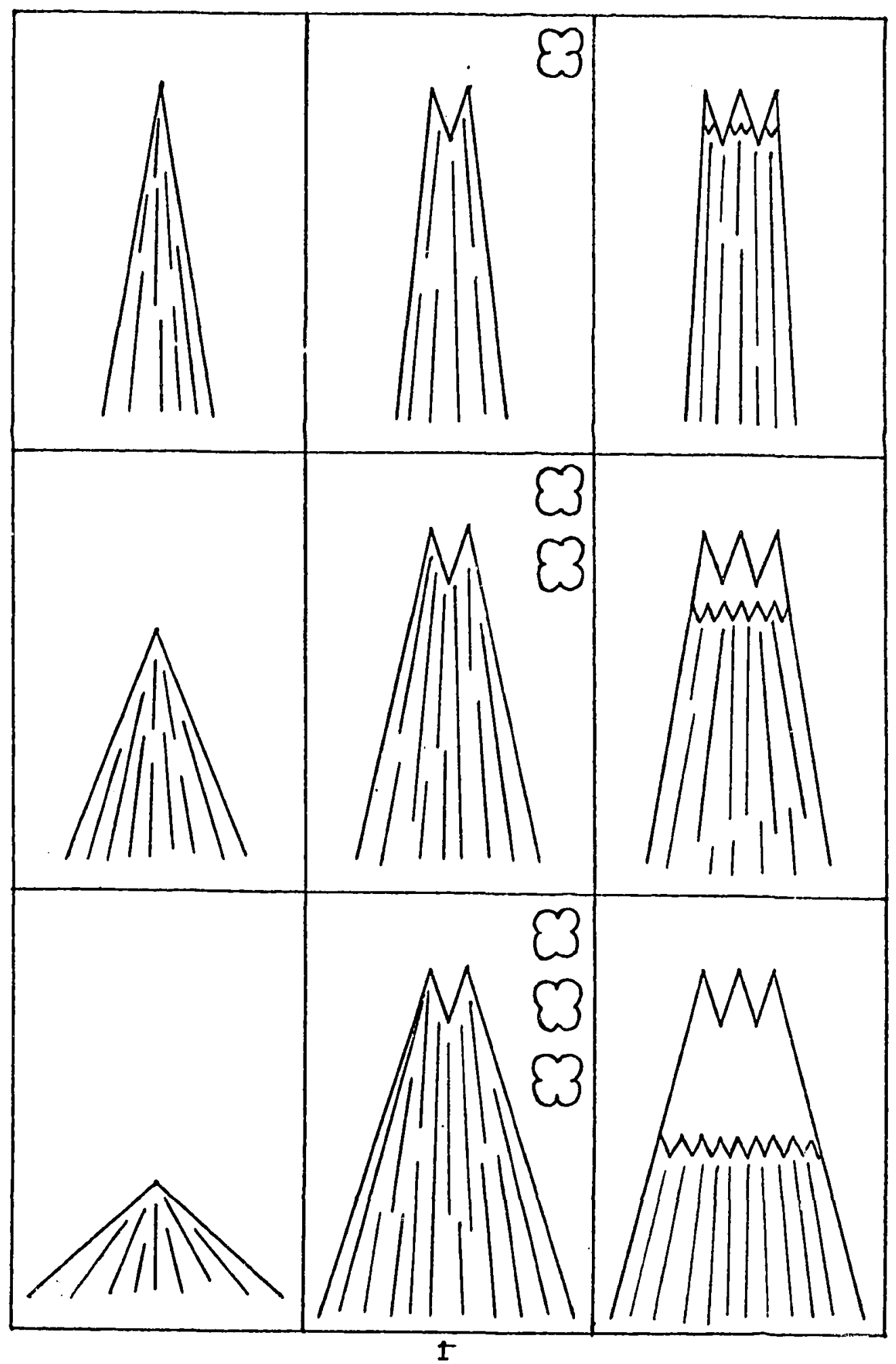




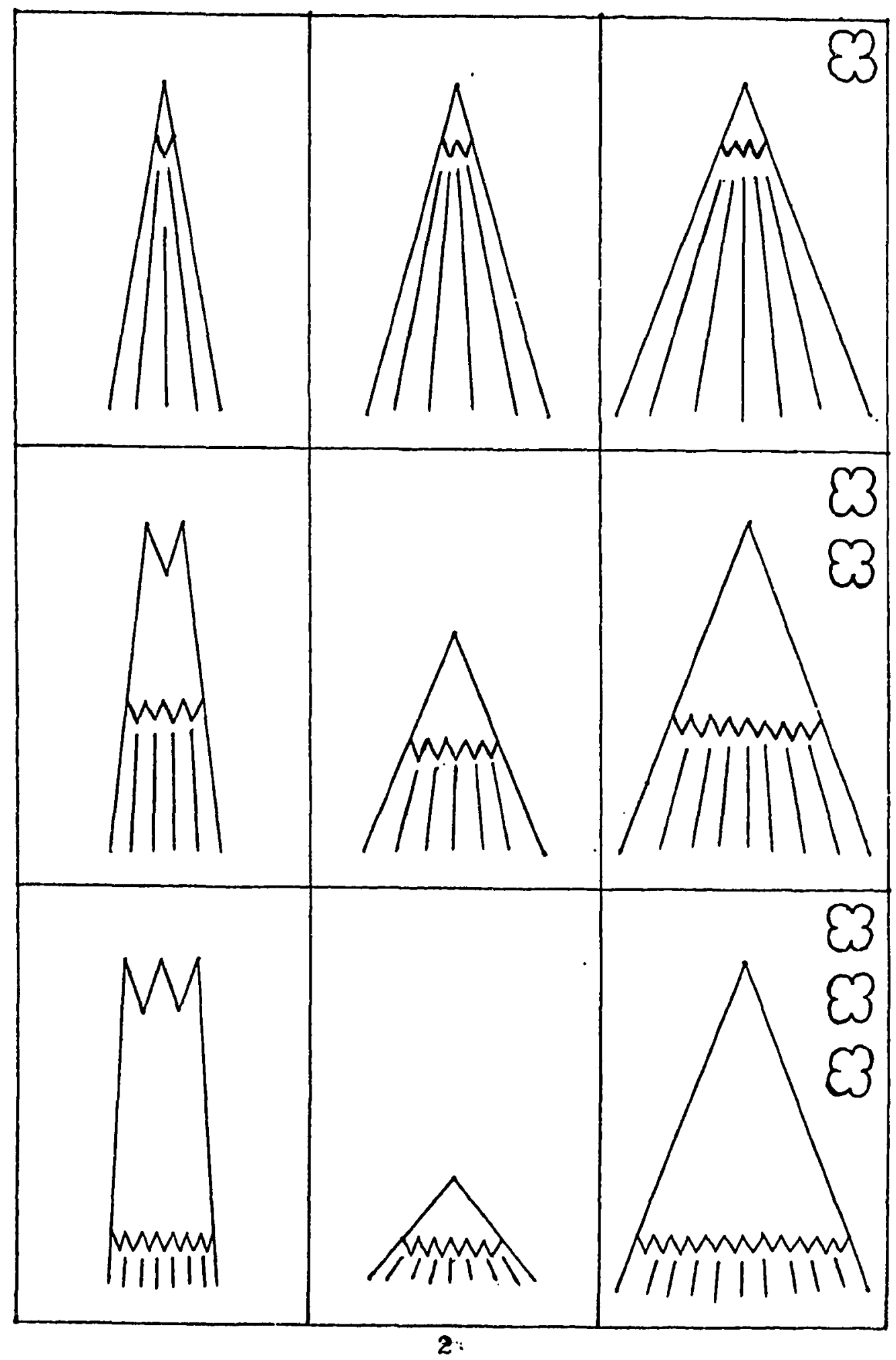




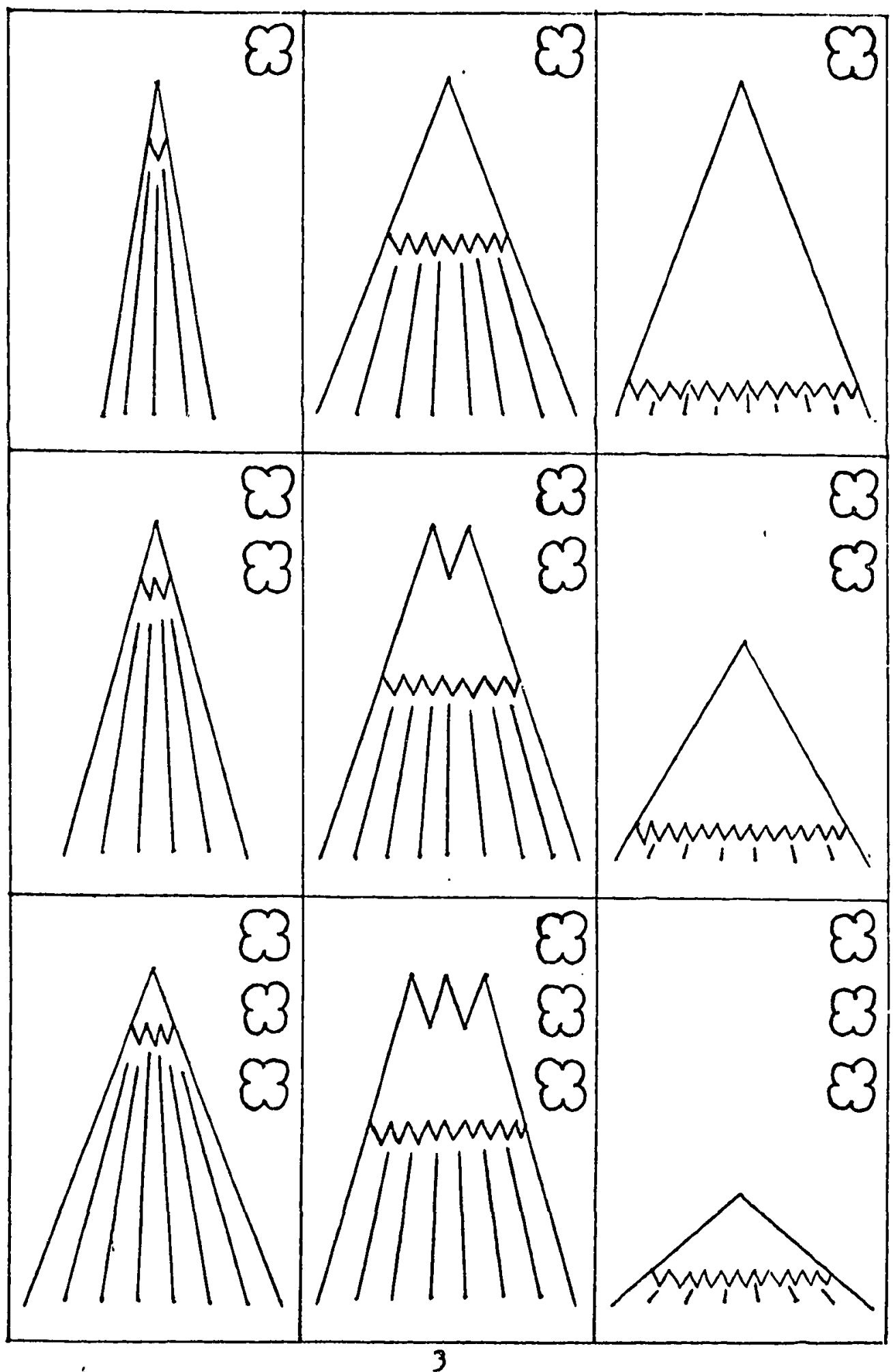









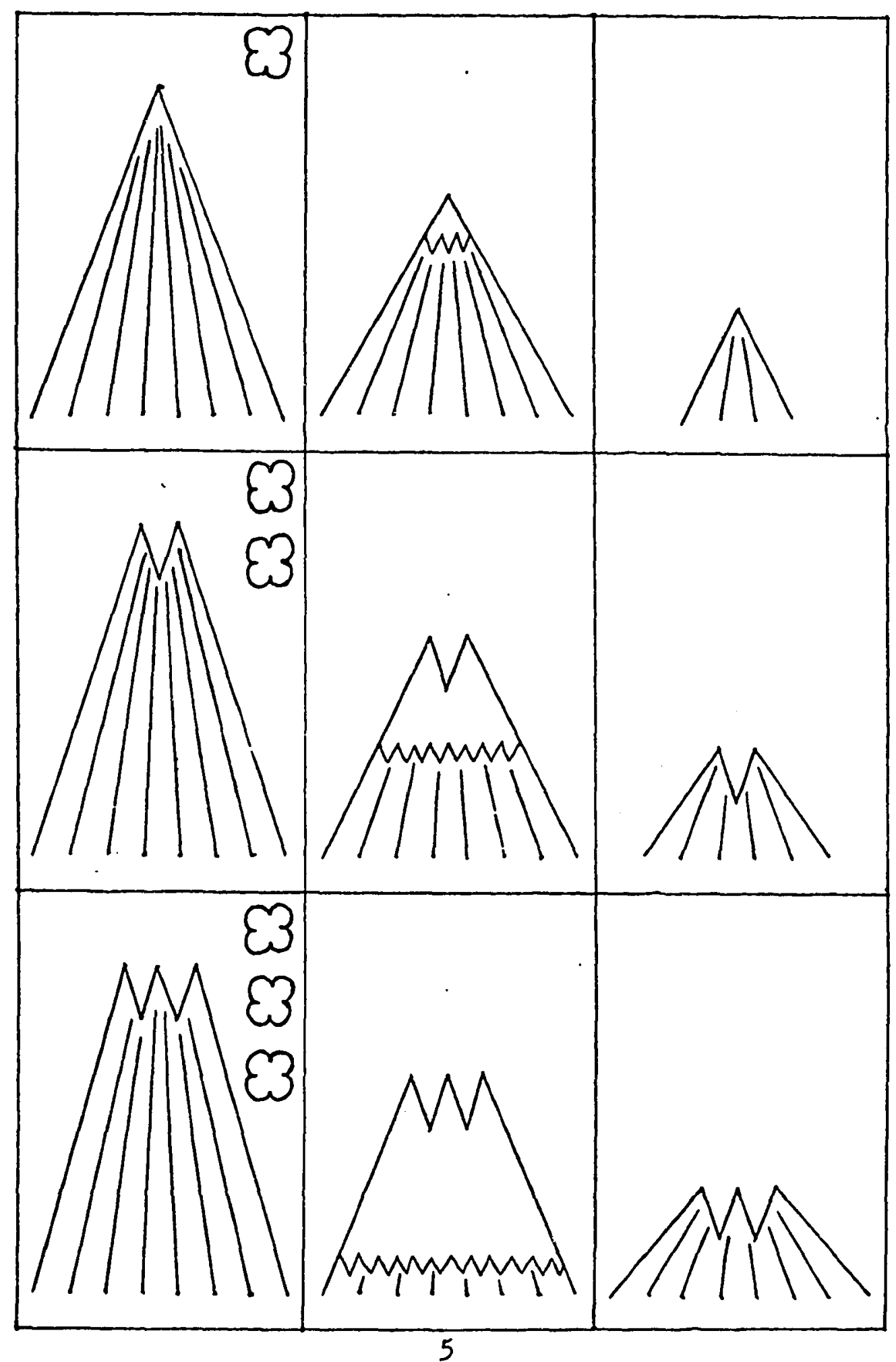




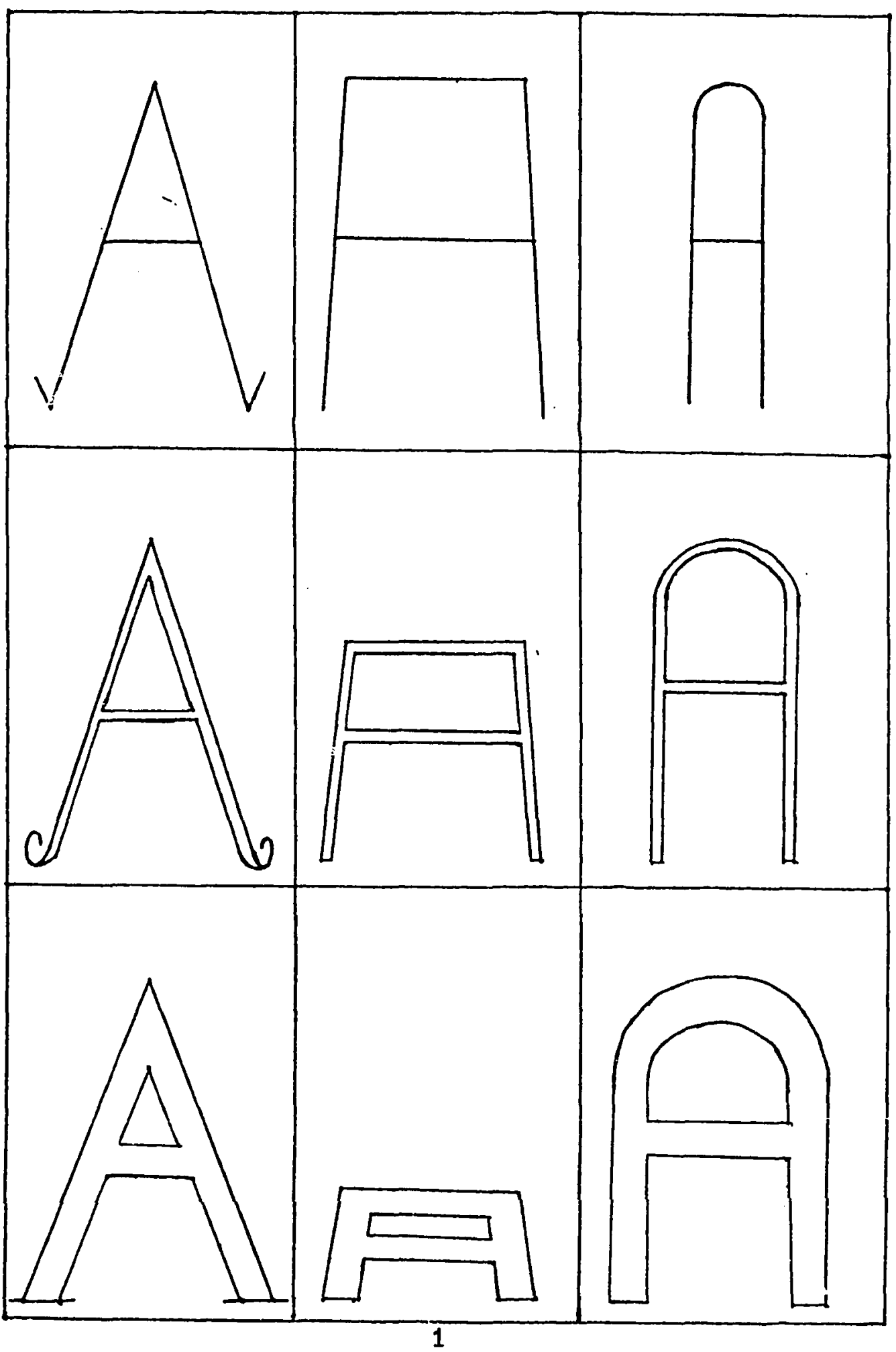




$$
\begin{array}{|l|l|l|}
\hline A & A & A \\
\hline A & A & A \\
\hline A & A & A \\
\hline
\end{array}
$$




$$
\begin{array}{|l|l|l|}
\hline A & A & A \\
A & A & A \\
\triangle & A & A \\
\hline
\end{array}
$$


100

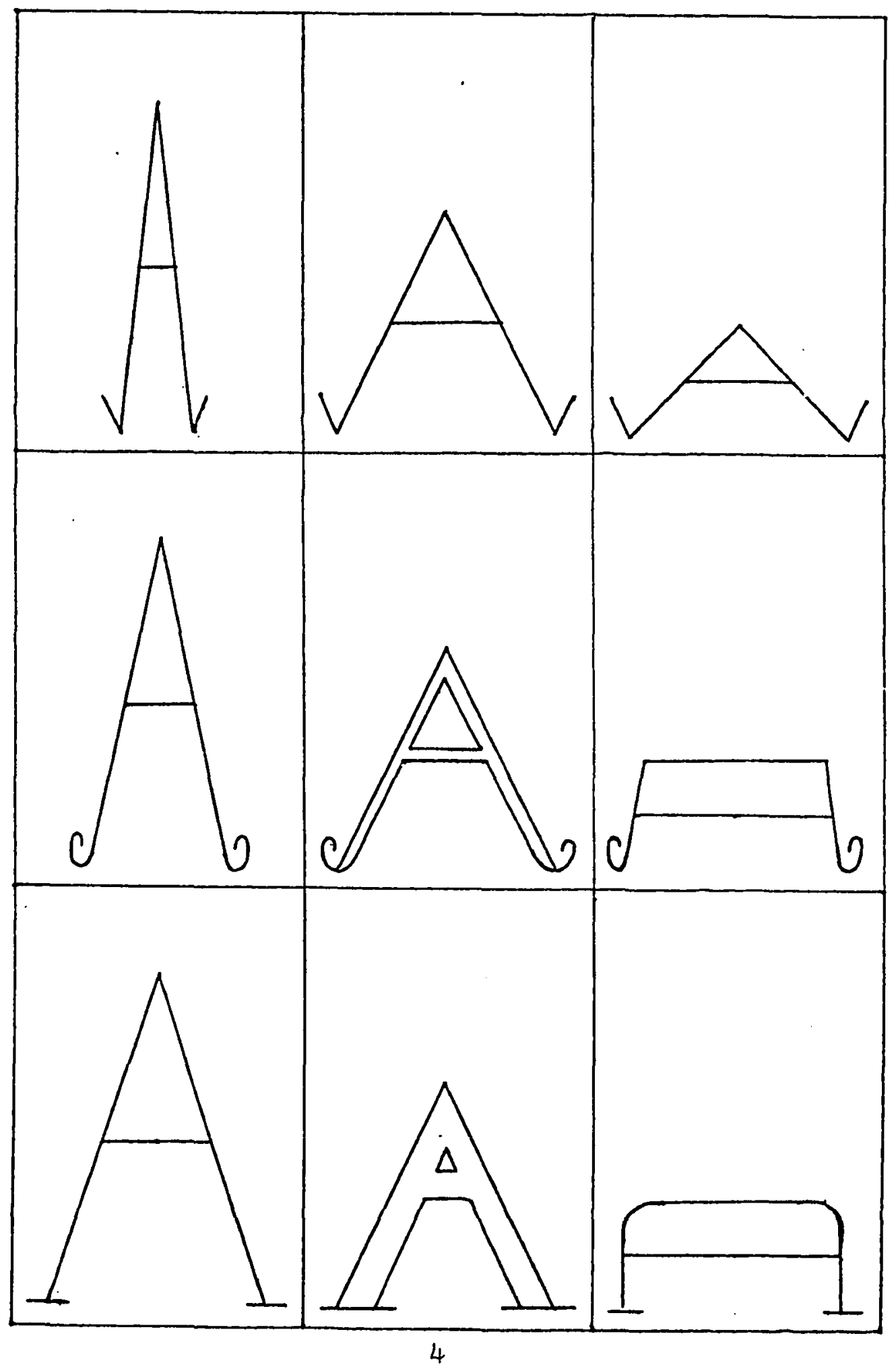




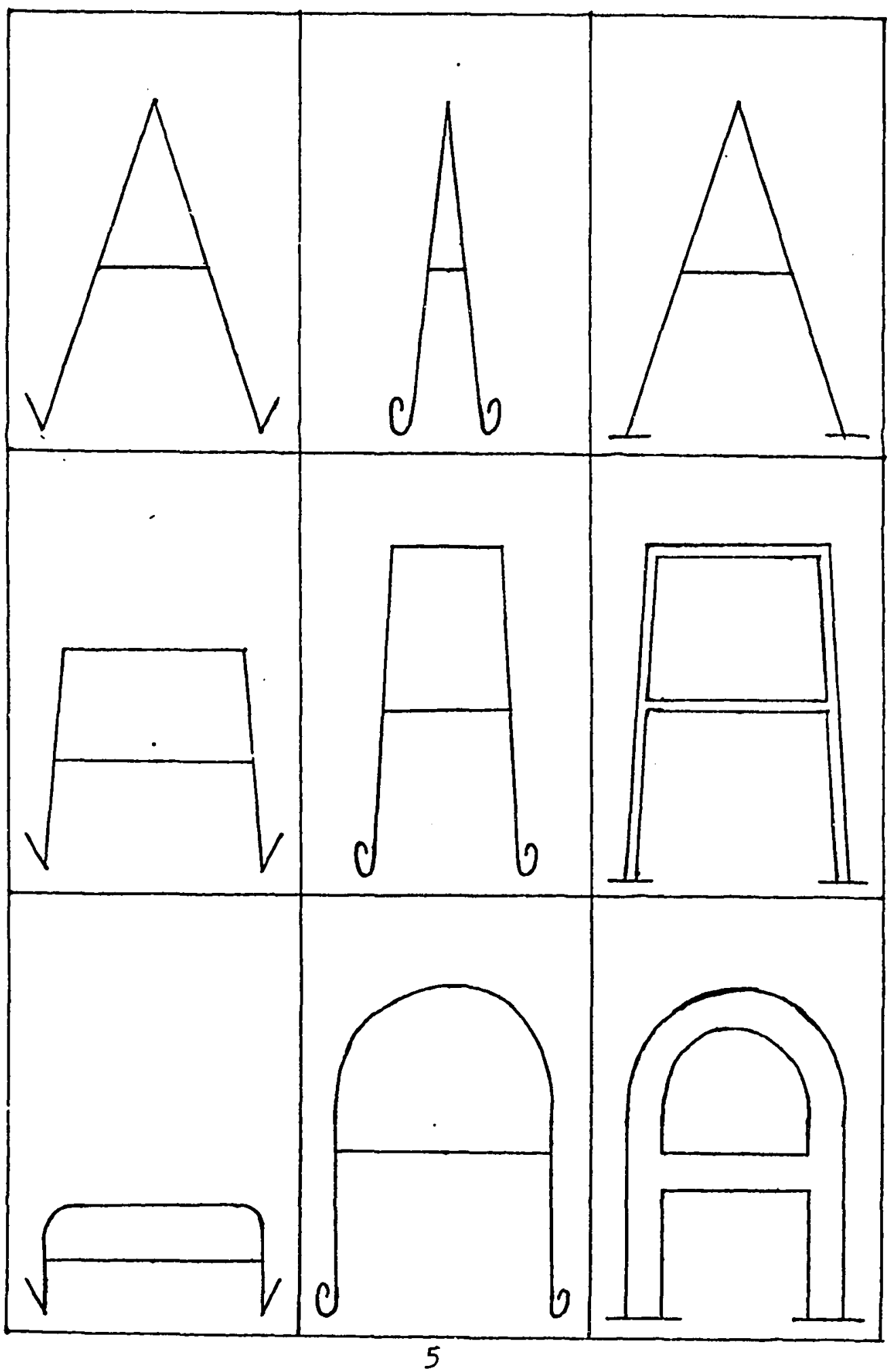




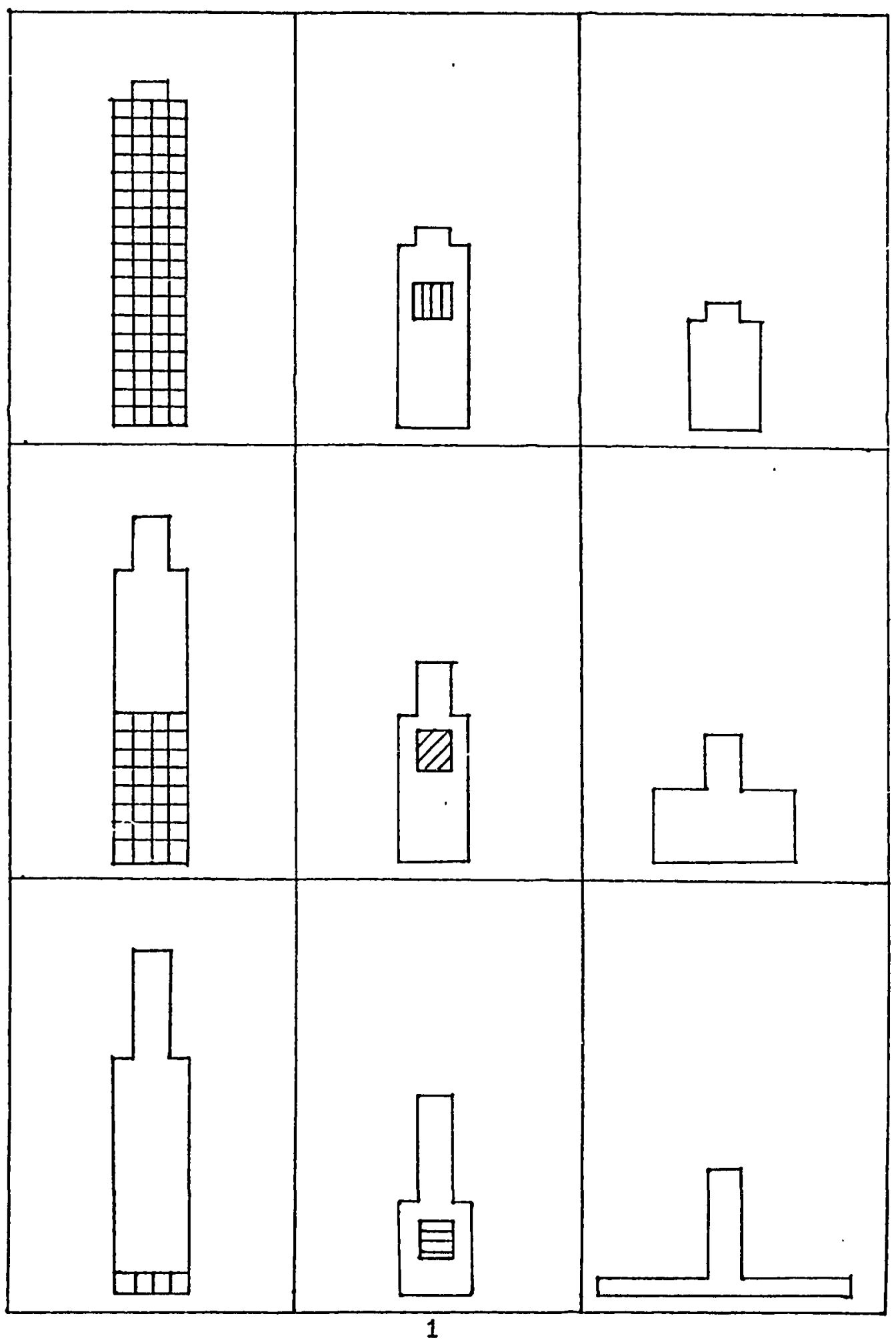




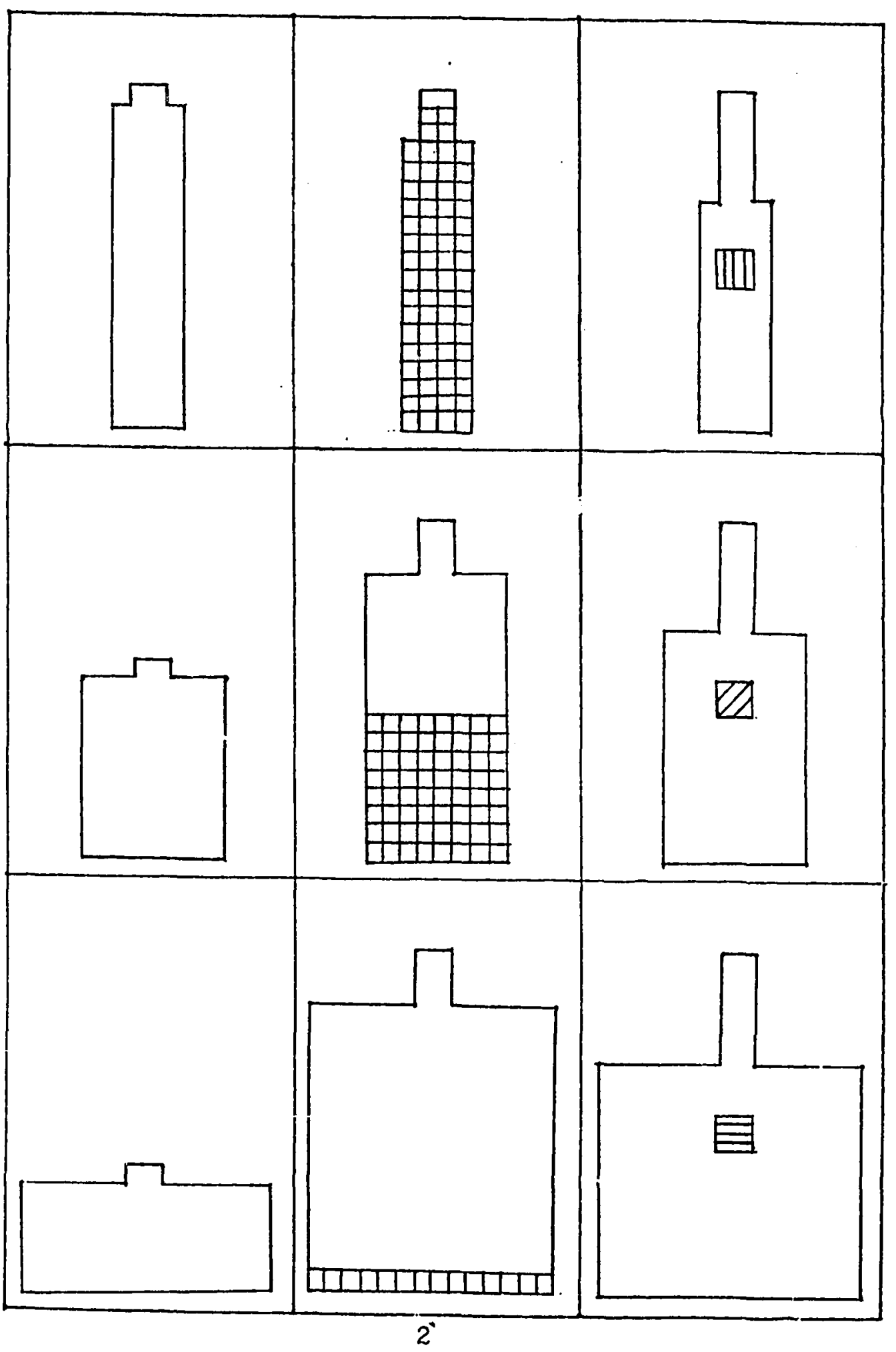




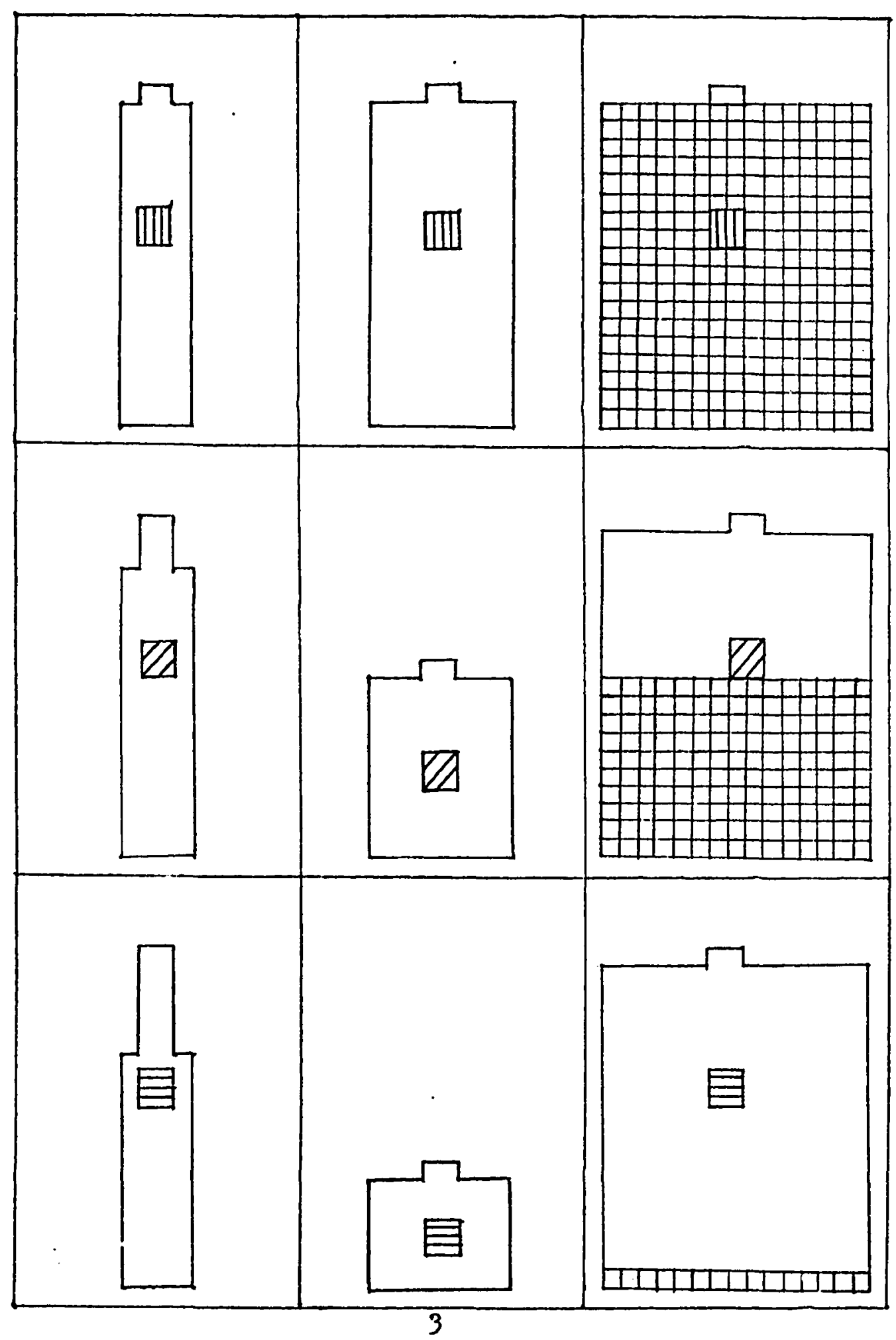




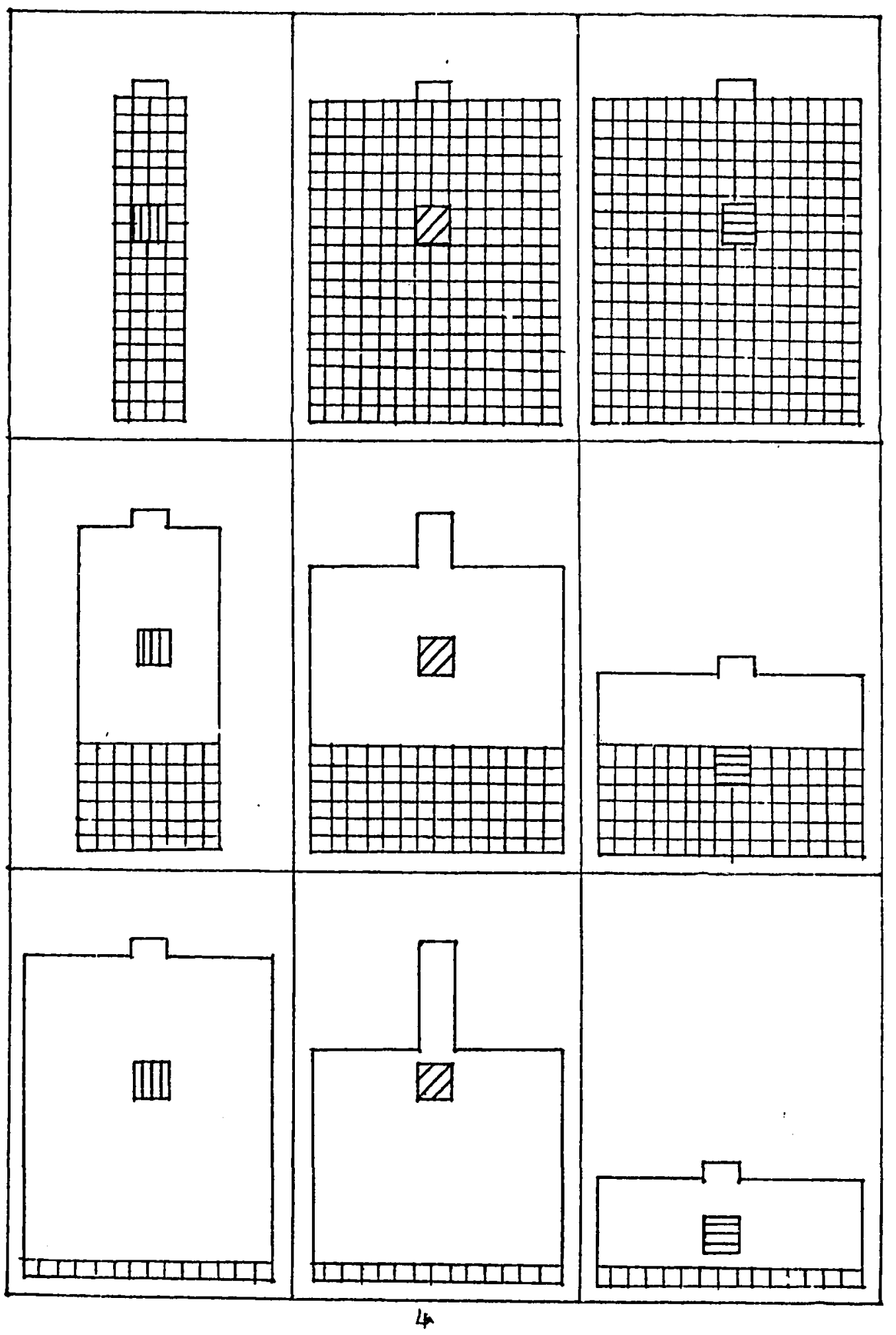




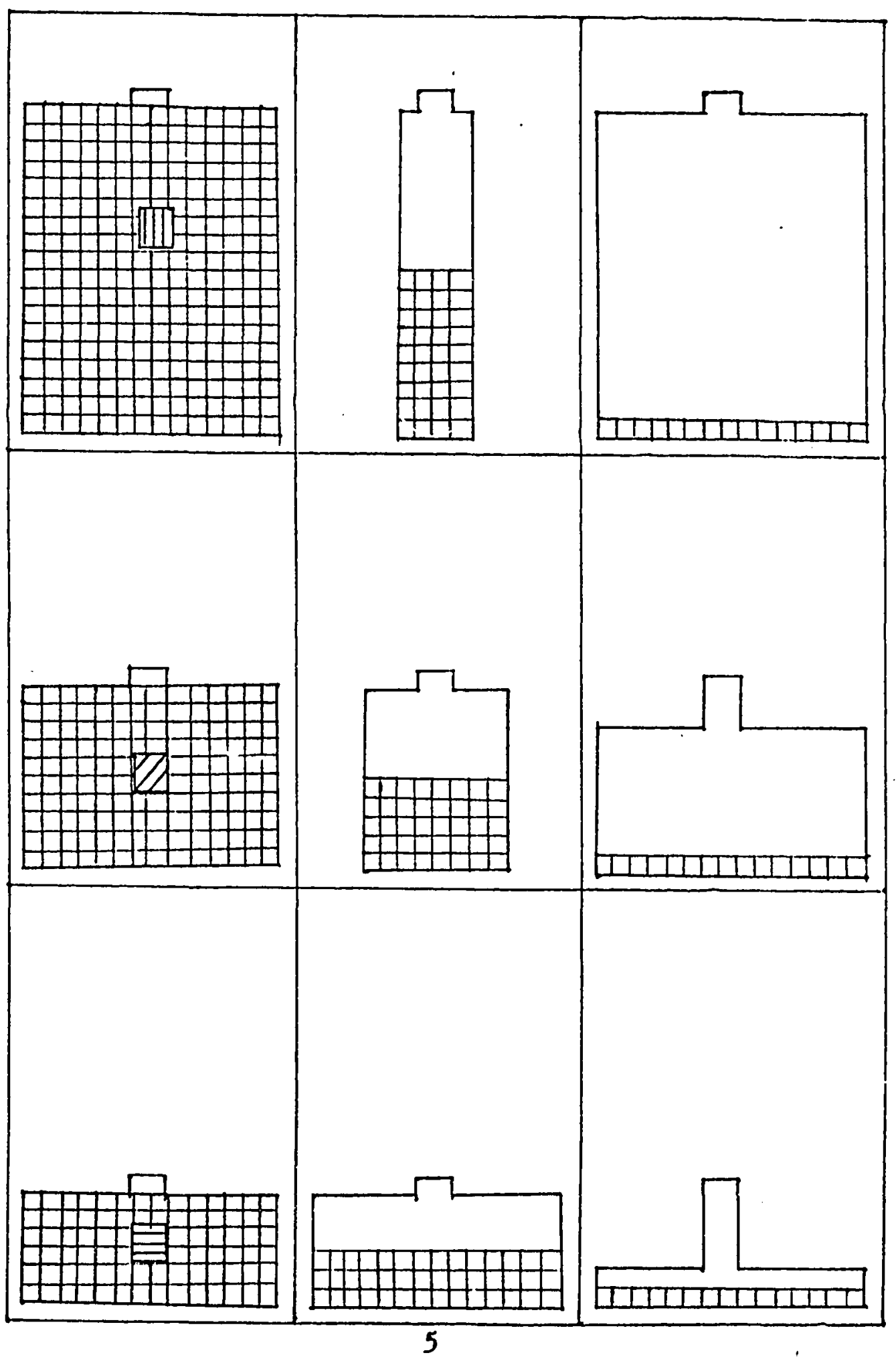




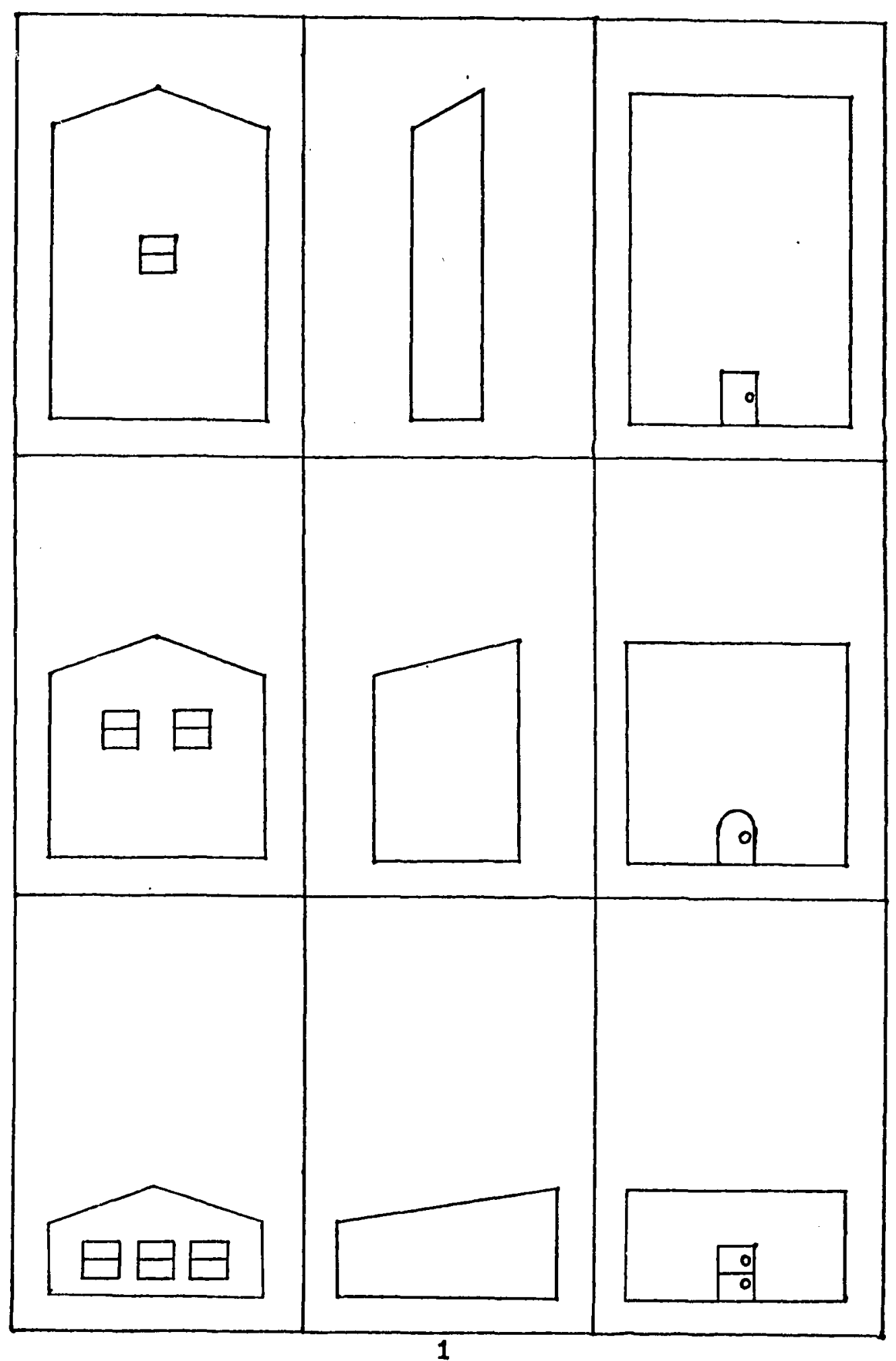




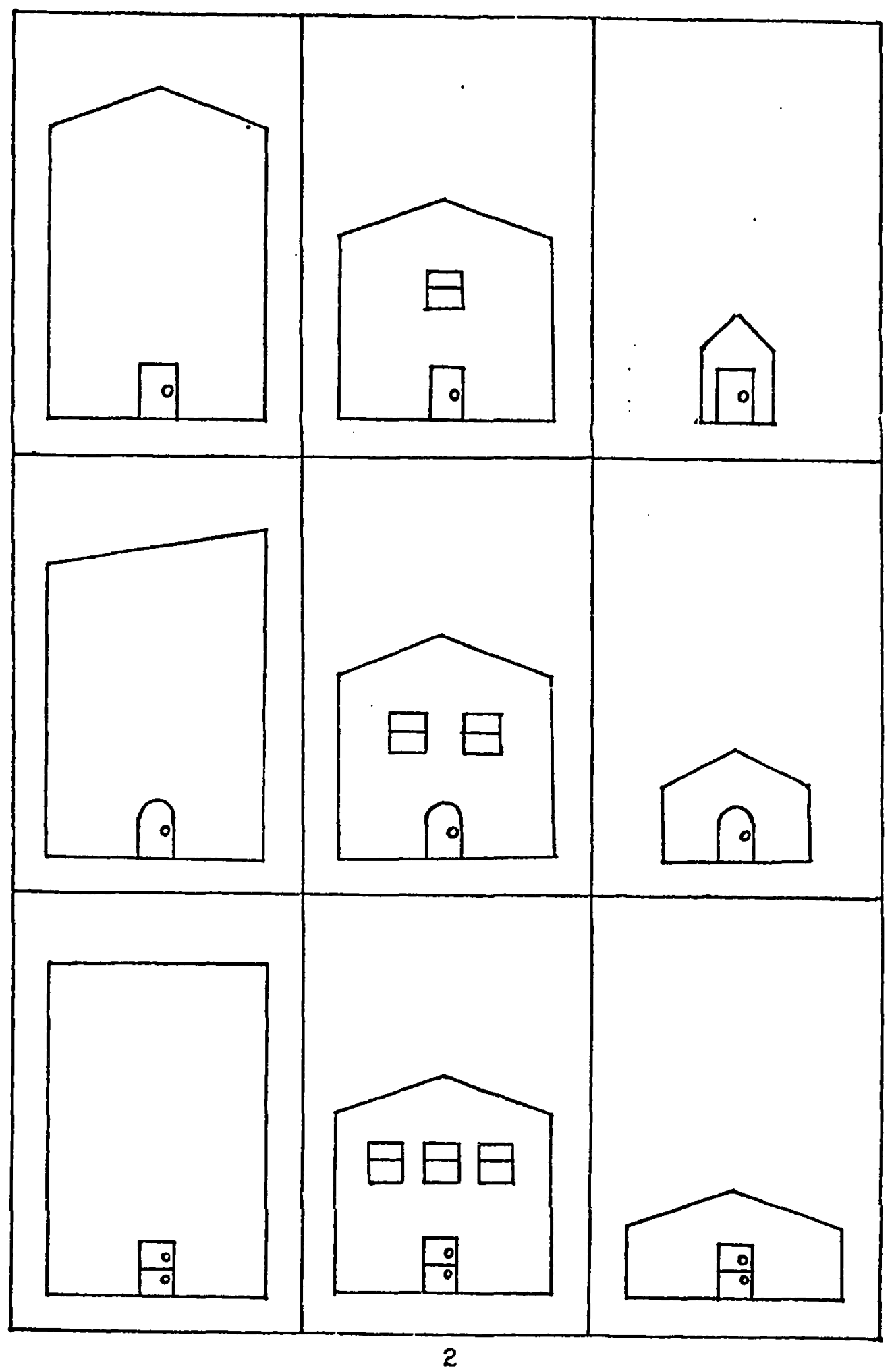




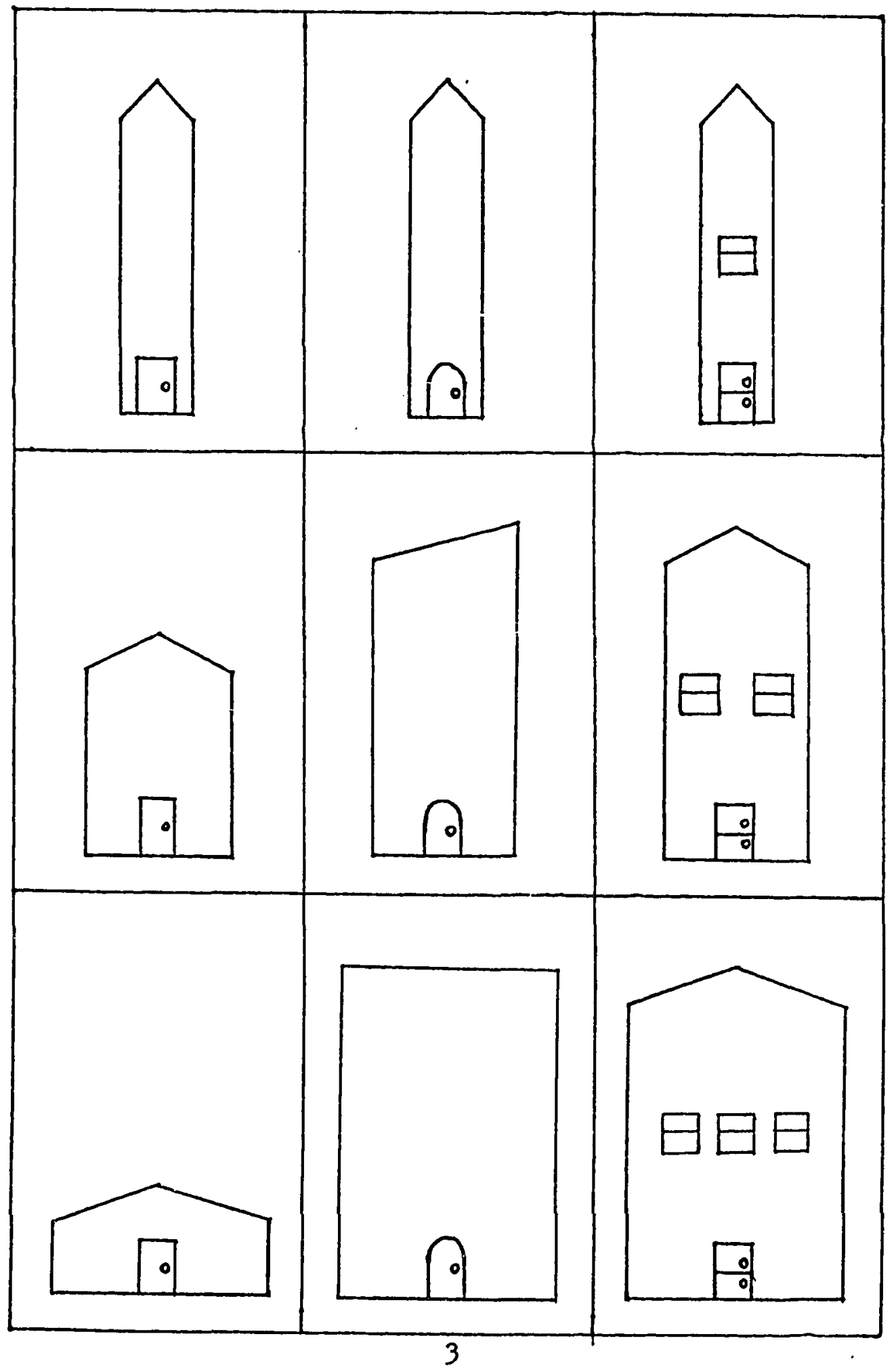




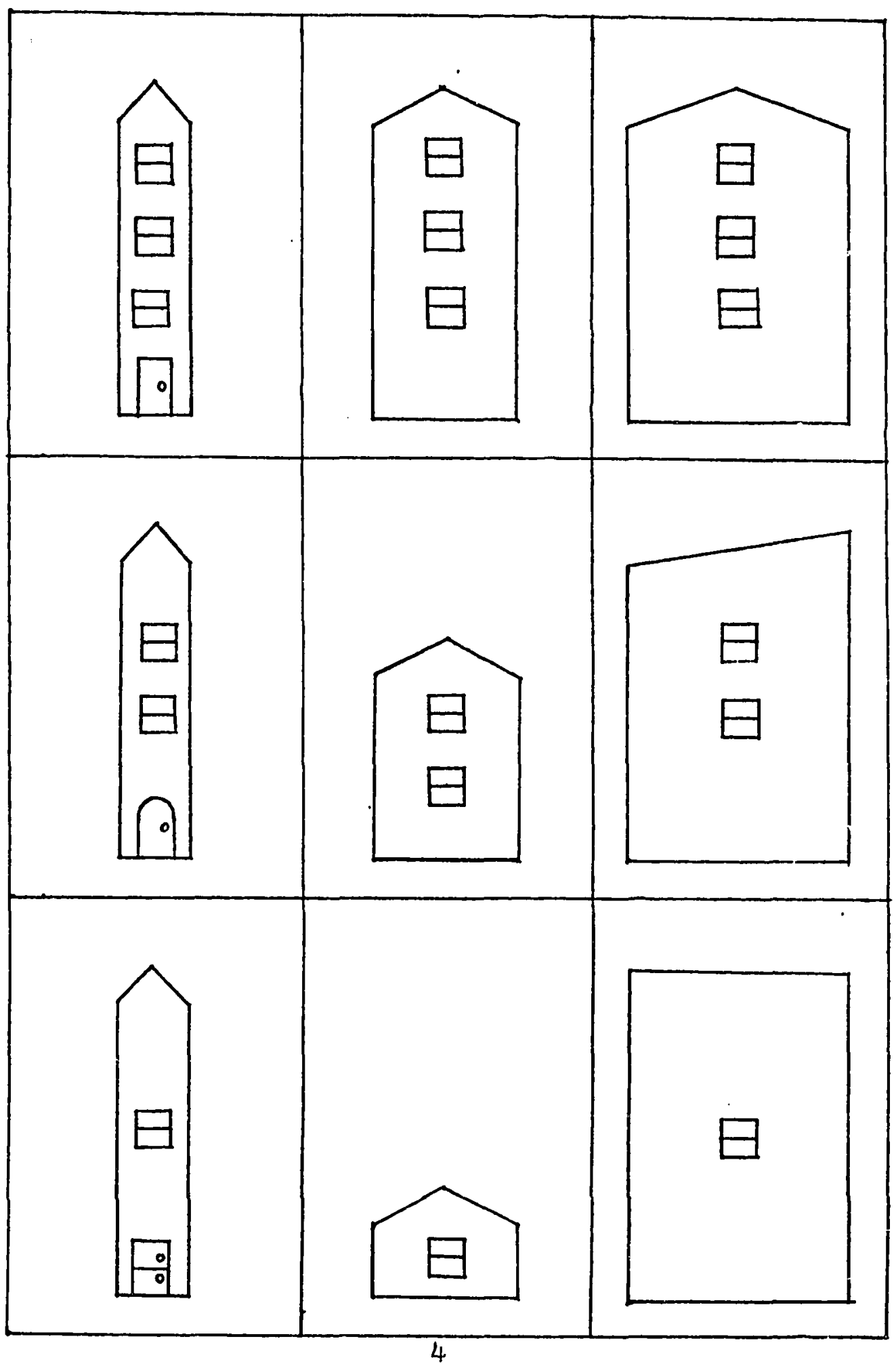




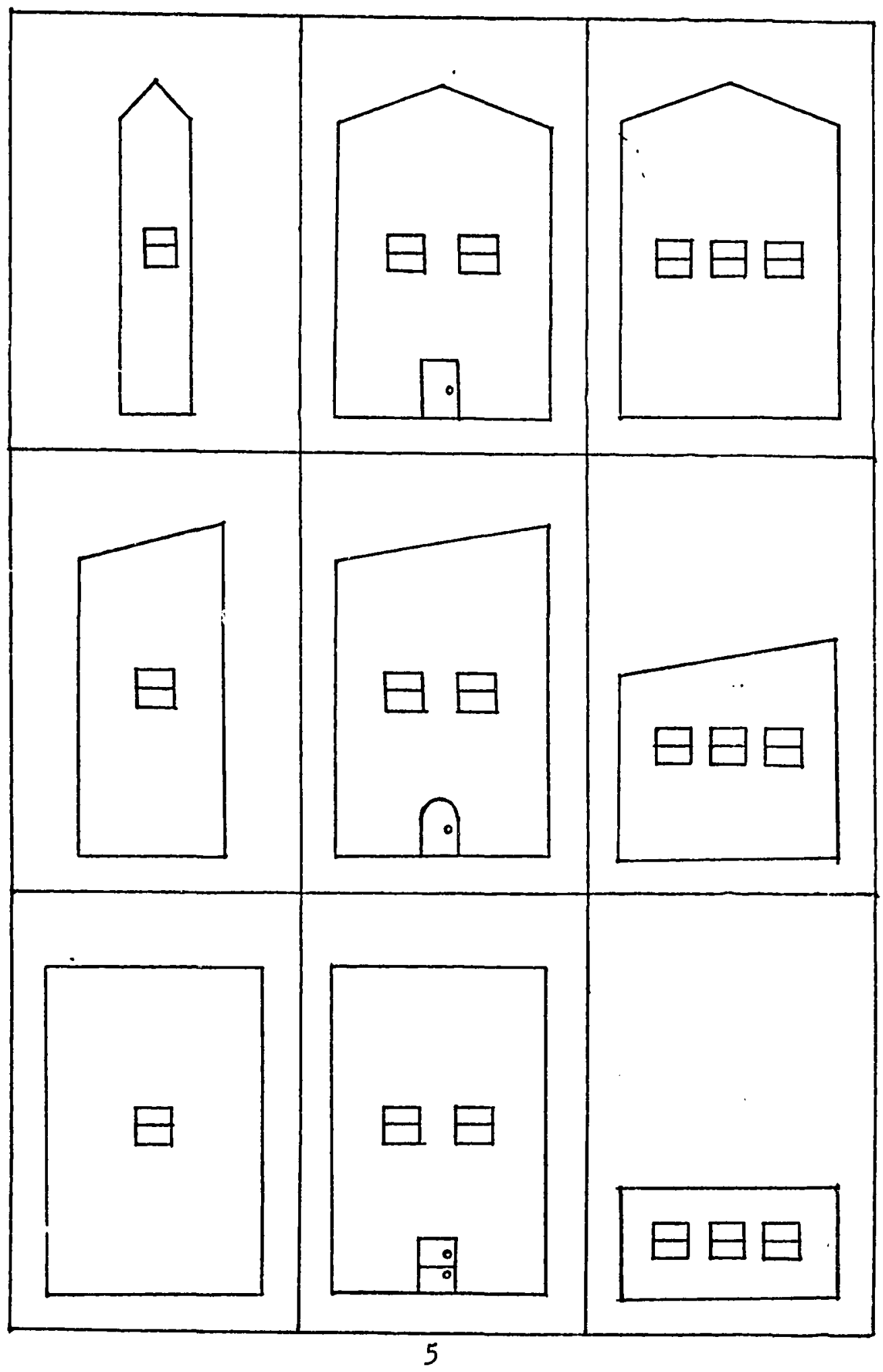

